\title{
Continuously driven phase separation: size distributions and time scales in droplet growth
}

\author{
Dissertation \\ zur Erlangung des mathematisch-naturwissenschaftlichen Doktorgrades \\ "Doctor rerum naturalium" \\ der Georg-August-Universität Göttingen \\ im Promotionsprogramm ProPhys \\ der Georg-August University School of Science (GAUSS)
}

\author{
vorgelegt von \\ Martin Rohloff \\ aus Achim \\ Göttingen, 2015
}




\section{Betreuungsausschuss}

Prof. Dr. Jürgen Vollmer, Dynamik komplexer Fluide, Max-Planck-Institut für Dynamik und Selbstorganisation (MPI DS)

Prof. Dr. Stephan Herminghaus, Dynamik komplexer Fluide, Max-Planck-Institut für Dynamik und Selbstorganisation (MPI DS)

Prof. Dr. Marcus Müller, Institut für theoretische Physik, Universität Göttingen

\section{Mitglieder der Prüfungskommision}

Referent: Prof. Dr. Marcus Müller, Institut für theoretische Physik, Universität Göttingen

Korreferent: Prof. Dr. Jürgen Vollmer, Dynamik komplexer Fluide, Max-PlanckInstitut für Dynamik und Selbstorganisation (MPI DS)

Weitere Mitglieder der Prüfungskommision:

Prof. Dr. Andreas Tilgner, Institut für Geophysik, Universität Göttingen

Prof. Dr. Andreas Dillmann, Deutsches Zentrum für Luft- und Raumfahrt (DLR), Göttingen

Prof. Dr. Stephan Herminghaus, Dynamik komplexer Fluide, Max-Planck-Institut für Dynamik und Selbstorganisation (MPI DS)

Dr. Claus Heussinger, Institut für theoretische Physik, Universität Göttingen

Tag der mündlichen Prüfung: 16.07.2015 


\section{Preface}

Being asked about the topic of my thesis, I would now say it is about many things, ranging from a model of rainfall over the synthesis conditions for highly monodisperse quantum dots to geyser or volcanic eruptions, or on the question why big aggregates get bigger and a mechanism to suppress inequalities in size. Or it is just the investigation of a fundamental question in phase separation. These relations and fruitful connections to many other fields of physics became obvious to me during my $\mathrm{PhD}$. They were a source of fascination and inspired me to study the underlying mechanisms by applying temperature protocols to mixtures of two fluids, giving jobs to the computer and staring at equations on a piece of paper.

On the journey to the present thesis many people joined me for longer or shorter times, giving me advice and support.

First of all I would like to express my deep gratitude to my supervisor Jürgen Vollmer for the many years we worked together. His extensive comments when asked for advice often provided a fresh view, if something was stuck. I learned a lot from the way he does physics.

I would like to thank Marcus Müller for being a referee of my thesis.

For the daily life doing experiments, improving and extending the experimental setup I am grateful to many people: To Wolf Keiderling and Christian Jacob and the workshop for realizing new ideas and changes of the setup. To Holger Nobach, Matthias Schröter, Christoph Gögelein and Paul Steffen for enlightening discussion about measurement techniques. Kris Hantke helped me a lot in questions concerning optics, whereas Markus Benderoth supported me concerning chemistry. I am very grateful to Thomas Eggers who gave me support in any IT question and solved my computer problems; in many cases almost instantly.

Especially I am grateful for the collaborations with Julian Vogel and Marcel Ernst, who both joined my project for their Bachelor theses, and with Darcy Jacobson, who joined as a RISE summer student.

I appreciate fruitful discussions with Alain Pumir, Michael Wilkinson, Lucas Goering, Bernard Cabane, Markus Abel and Nicolas Rimbert.

I am grateful to Jürgen, Lucas and Friederike for feedback on the manuscript.

I would like to express my gratitude to Stephan Herminghaus, for the opportunity to work in his inspiring department. I thank all the people in the department of complex fluids for their company, especially the groups of Jürgen's and Marco's. I enjoyed the work and leisure time in particular on the retreats. 
I would also thank Tobias Lapp for the wonderful collaboration on phase separation in binary mixture before I started with my $\mathrm{PhD}$. He got me interested in this topic and I am happy that I could continue his work.

Finally, I would like to thank my family Friederike, Sophia, Alma and David and all the people who accompanied me in the recent years. 


\section{Contents}

Preface

1 Introduction 1

1.1 Motivation . . . . . . . . . . . . . . . . 1

1.2 Phase transitions and demixing . . . . . . . . . . . . . . 3

1.3 Ostwald ripening and Lifshitz-Slezov-Wagner theory . . . . . . . . 7

1.4 Ripening with material flux . . . . . . . . . . . . . . 9 9 9

1.5 Collisions and sedimentation . . . . . . . . . . . 10

1.6 Outline ............................... 12

2 Experimental techniques and methods $\quad 15$

2.1 Experimental setup for investigating binary fluid demixing . . . . . 15

2.2 Phase diagrams of binary fluid mixtures . . . . . . . . . . . 17

2.3 Driving the demixing by temperature ramps . . . . . . . . . . . . 19

2.3.1 Calculating $\xi$ from the form of the phase-coexistence curve . 19

2.3.2 Calculating temperature ramps . . . . . . . . . . . 22

2.3.3 Influence of phase-diagram representation on driving $\xi \ldots \ldots 22$

2.3.4 Calculating $\xi$ from measured temperature ramps . . . . . . . 23

2.4 Turbidity measurements . . . . . . . . . . . . . . . . 25

2.4.1 Setup for turbidity measurements . . . . . . . . . . 25

2.4.2 From turbidity to oscillation periods . . . . . . . . . . . 26

2.5 Measuring size distributions . . . . . . . . . . . . . 26

3 Numerical methods $\quad 29$

3.1 Dimensionless units . . . . . . . . . . . . . . . . . . . 30

3.1 .1 Time and length scales . . . . . . . . . . . . . 30

3.1.2 Dimensionless equation . . . . . . . . . . . . . 31

3.2 Initial distributions . . . . . . . . . . . . . . . . . . . . 32

3.2 .1 Uniform distribution . . . . . . . . . . . . . . . . . . . . . . . . . . . . . . .

3.2 .2 Bell-shaped distribution . . . . . . . . . . . . . 33

3.2 .3 Bimodal distribution . . . . . . . . . . . . . . . . 33

3.3 Integration scheme . . . . . . . . . . . . . . . 35 
4 Size Distributions $\quad 39$

4.1 Ripening with overall volume growth: numerical results . . . . . . . 39

4.2 Theory for large $k$ values . . . . . . . . . . . . . . . . . . . 43

4.2.1 Moments of the aggregate size distribution . . . . . . . 45

4.2.1.1 The evolution of $\left\langle R^{3}\right\rangle \ldots \ldots \ldots \ldots . \ldots 45$

4.2.1.2 The average aggregate radius $\langle R\rangle \ldots \ldots \ldots$

4.2.1.3 Deviation of $\langle R\rangle^{3}$ from $\left\langle R^{3}\right\rangle \ldots \ldots \ldots$. . . . . . 47

4.2.1.4 The variance of the distribution . . . . . . . . 48

4.2 .2 Reduced aggregate radius . . . . . . . . . . . . 49

4.2.2.1 Evaporation of aggregates . . . . . . . . . 50

4.2.2.2 Evolution of the reduced aggregate radius . . . . . 52

4.2.3 Evolution of the aggregate size distribution . . . . . . . . 53

4.2.3.1 Initial distribution, and its evolution based on eq. (4.19) 53

4.2.3.2 Accounting for broadening and shape changes . . . 55

4.2.3.3 Scaling of the centred moments of the size distribution 57

4.3 Size distributions in the experiment . . . . . . . . . . 58

4.3 .1 Experimental observations . . . . . . . . . . . . 59

4.3.2 Growth law for sedimenting droplets . . . . . . . . . . . 61

4.3.3 Evolution of the size distribution with sedimentation . . . . . 62

4.3.4 Comparison with theory . . . . . . . . . . . 63

4.4 Discussion and summary . . . . . . . . . . . . . 66

4.4 Theory ....................... 66

4.4 .2 Experiments . . . . . . . . . . . . . 68

5 Oscillation periods for constant ramp rates $\quad 69$

5.1 Experimental results for the oscillation period . . . . . . . . . . . 69

5.1 .1 Measuring the oscillation period . . . . . . . . . 69

5.1 .2 Influence of flow . . . . . . . . . . . . . 70

5.2 Calculating the oscillation period for constant $k$ values . . . . . . . . 73

5.2.1 Evolution of the radius of the largest droplets . . . . . . . . . 74

5.2.1.1 Growth by accretion of material provided by the constant driving, $\xi \ldots \ldots \ldots \ldots$. . . . . . . . 74

5.2.1.2 Growth by Ostwald ripening . . . . . . . . . 75

5.2.1.3 Growth by collection of smaller droplets . . . . . . 76

5.2.1.4 The bottleneck of droplet growth . . . . . . . . . 77

5.2 .2 Calculating the period $\Delta t \ldots \ldots \ldots \ldots \ldots$

5.3 Comparison to experimental data . . . . . . . . . . 80

5.3.1 Master plots for droplet tracking data . . . . . . . . . 80

5.3 .2 Master plots for turbidity data . . . . . . . . . . . . . 82

5.3 .3 Influence of flow . . . . . . . . . . . . . . . . 82 
5.4 The relevance of Ostwald ripening: A quantitative test . . . . . . . 83

5.4.1 Values of $\alpha$ for binary mixtures . . . . . . . . . . . . . 84

5.4.2 Bottleneck radius . . . . . . . . . . . . . . . . . 84

5.4.3 Temperature dependence of $\Delta t \ldots$. . . . . . . . . . . 86

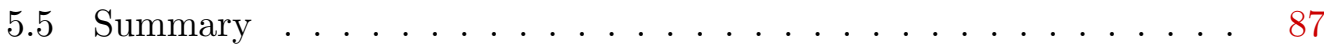

6 Periodic Driving $\quad 89$

6.1 Phase locking and Arnold tongues . . . . . . . . . . . . . . . 89

6.1.1 The state variables and their evolution equations . . . . . . 89

6.1.2 Dimensionless units ................. . . 90

6.1.3 Oscillation period for $A=0$............. 90

6.1.4 Oscillation period for $A>0$.............. 91

6.1.5 Phase locking and Arnold tongues ............. . 94

6.2 Experimental results . . . . . . . . . . . . . . 96

6.2.1 Realization of temperature ramps . . . . . . . . . . 96

6.2.2 1:1 Arnold tongues .................. . . 97

6.2.3 1:2 Arnold tongues .................... 99

6.2.4 1:3 Arnold tongues . . . . . . . . . . . . . . . . 99

6.2.5 5:1 Arnold tongues . . . . . . . . . . . . . . . . 100

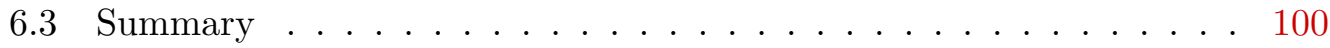

7 Discussion 103

7.1 Synthesis of monodisperse colloids and nano-particles . . . . . . . 103

7.1.1 Estimate values of $k$. . . . . . . . . . . . . . 103

7.1.2 Shape of the distributions and size focussing . . . . . . . 106

7.2 Rain Formation . . . . . . . . . . . . . . . . . . 106

7.2.1 Growth of cloud droplets: estimating the value of $k$. . . 106

7.2.2 Estimates of $\Delta t$ and the bottleneck radius . . . . . . . . 108

7.2.3 Periodic driving. . . . . . . . . . . . . . . . 108

7.3 Geysers and lake eruptions .................. . . 109

7.4 Summary .................... . . 110

8 Conclusion 111

Appendix: Material Constants $\quad 115$

A.1 Isobutoxyethanol and water . . . . . . . . . . . . 115

A.1.1 Density .................... 115

A.1.2 Viscosity ..................... 117

A.1.3 Diffusion coefficient .................. 118

A.1.4 Interfacial tension . . . . . . . . . . . . . . . 118

A.1.5 Molar volume .................. . 119 
A.2 Methanol and hexane . . . . . . . . . . . . . . . 119

A.2.1 Density . . . . . . . . . . . . . . . . . . . . . 119

A.2.2 Viscosity . . . . . . . . . . . . . . . . . . . . . 120

A.2.3 Diffusion coefficient . . . . . . . . . . . . . . 120

A.2.4 Interfacial tension . . . . . . . . . . . . . . . 121

A.2.5 Molar volume . . . . . . . . . . . . . . . . 121

Bibliography 


\section{Introduction}

\subsection{Motivation}

In mixtures phase separation arises when temperature, pressure or concentrations of mixtures are changed, such that a new macroscopic phase emerges (Stanley, 1971; Yeomans, 1992). Typically the domains of the new phase take the form of small droplets, bubbles or solid particles immersed in a fluid or solid matrix. Initially the domains are small, but under equilibrium conditions their average size grows while their number diminishes at the same time (Ostwald ripening)(Becker (1985, pp. 58), Ratke and Voorhees (2002)). In many natural processes as well as technical applications the thermodynamic parameter controlling the phase separation changes continuously, rather than abruptly. Hence, it provides a continuous driving of the demixing and domain growth by the driving is competing with ripening.

In the present thesis I will address the evolution of the size distribution of the domains by experimental, numerical and theoretical studies. The results will be interpreted from the perspective of their implications for the description of the formation of steady rain (Jameson and Kostinski, 2002) and recipes for the synthesis of colloidal particles. In the latter a chemical precursor reaction or external addition of material provides the monomers for the growth of particles (cf. the review of Sowers et al. (2013)).

Other examples, where continuously driven phase separation emerges as a key ingredient of phase separation are hot-water geysers (Ingebritsen and Rojstaczer, 1993; Toramaru and Maeda, 2013) and cold-water geysers (Han et al., 2013), as well as lake (Zhang, 1996; Zhang and Kling, 2006) and volcano (Wylie et al., 1999; Cashman and Sparks, 2013) eruptions. In these cases there are episodic eruptions, that arise because gravity acts like a feedback that enhances bubble growth, in the late stages of bubble growth. For instance the presence of bubbles in the conduit of a cold water geyser lowers the solubility of $\mathrm{CO}_{2}$ at the bottom, thus increasing the production of bubbles. This feedback mechanism leads to a runaway and resets the system. We denote this as episodic precipitation.

Remarkably, rain formation in clouds can also be considered as an instance of episodic precipitation. Here atmospheric vapour condenses continuously due to adiabatic cooling of rising humid air parcels. Apart from pressure and temperature changes that drives the phase separation, also entrainment of dry air at the border of clouds influences the growth of cloud droplets (see de Rooy et al., 2013; Tölle and Krueger, 


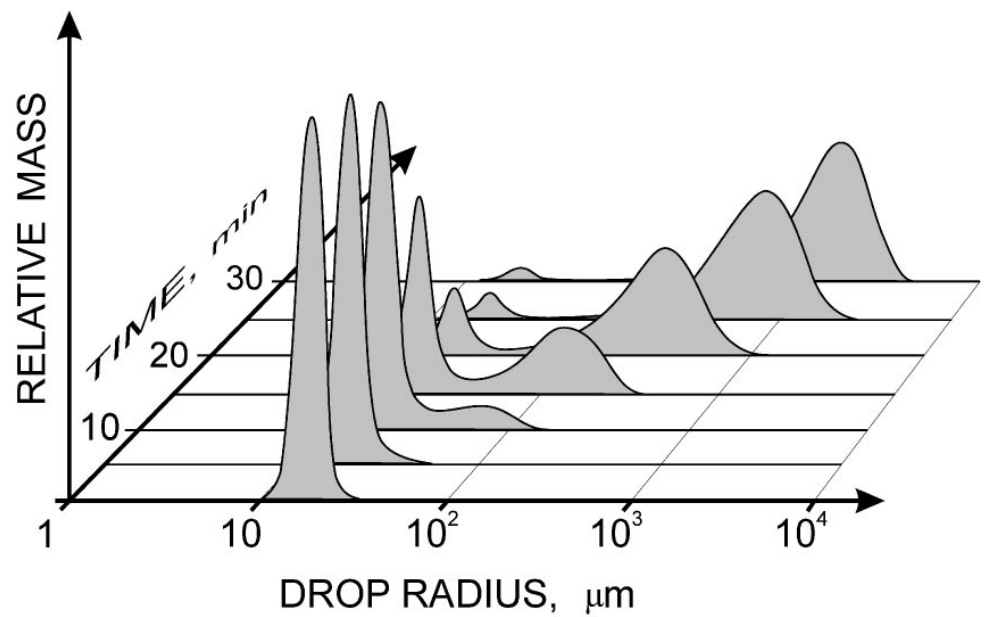

Figure 1.1: Illustration of the evolution of the size distribution in a cloud during the onset of the collision-coalescence process. Reprinted from Shaw (2003).

2014, for recent discussions). All these processes interact to set the time scale for rain formation. I will address the implications of my work for episodic eruptions, colloid synthesis and clouds in chapter 7 .

These phenomena have in common that a steady flux of second phase material is caused by the continuously driven phase separation. The flux first rises supersaturation and after nucleation of second phase particles, it leads to growth in the presence of the material source. Depending on the source strength a rich crossover behaviour from coarsening to size focussing emerges in the evolution of the size distribution. Precipitation emerges when aggregates, i.e. droplets, bubbles or solid particles that are immersed in a fluid, grow to a size where their motion is affected by buoyancy. At this point their motion changes from Brownian diffusion to Stokes settling, and the probability for collisions with other aggregates increases dramatically. As a consequence aggregate growth is boosted (Houghton, 1959; McGraw and Liu, 2003; Grabowski and Wang, 2013), collective effects emerge in their motion (Cau and Lacelle, 1993; Kalwarczyk et al., 2008; Stevens and Feingold, 2009; Woods, 2010), and virtually all volume condensed on the aggregates is precipitating out of the fluid in a finite time (Cau and Lacelle, 1993; Aarts et al., 2005; Kostinski and Shaw, 2005). In fig. 1.1 Shaw (2003) provides a sketch of the evolution of the droplet-size distribution in a cloud. This figure allows me to highlight fundamental questions in cloud physics that are the topic of the present thesis in a broader context. The narrow peak at small radii $(\sim 20 \mu \mathrm{m})$ represents cloud droplets, that form a stable non precipitating cloud. They behave like Brownian particles and are not (yet) affected by gravity. Until this size the diffusive growth of droplets shares features observed in the size focussing of particle-size distributions in colloid synthesis (Wallace and Hobbs, 2006; 
Clark et al., 2011). How does a material source change the coarsening behaviour of a polydisperse distribution? Due to the material flux of condensing vapour the droplets in the first peak grow: the peak shifts slightly to the right. At some point the largest droplets in the tail of the distribution start to feel gravity. Subsequently, they rapidly take over the distribution and form a second peak of rain droplets, that eventually fall out as precipitation. To understand this crossover one really has to look at the tail of the distribution of large cloud droplets. Clark et al. (2011) have recently established a description of the evolution of the first two moments of the size distribution. However, this is not sufficient for the clouds where particles in the tail of the distribution play such an eminent role (Kostinski and Shaw, 2005). I will therefore provide a theoretical description for the full distribution and all of its moments.

Recalling episodic precipitation of geysers one can imagine that once the droplets of a cloud precipitated a new life cycle starts for the air that formed the cloud. This immediately rises the question of the emerging time scale in episodic precipitation. At what time will it rain next when the driving persists?

In the remaining part of this introduction I provide the basic concepts and ideas collected from different fields of physics, that will be combined and extended in the following chapters to give a consistent picture of size distributions and time scales in droplet growth for continuously driven phase separation.

\subsection{Phase transitions and demixing}

Phase diagrams are commonly used to describe phase transitions and phase separation. They are is a nice graphical means to indicate the state of a mixture by a phase coexistence curve or binodal line. In the present thesis I deal with mixtures of two components and mainly with temperature induced phase separation, thus only binary phase diagrams in the temperature $(T)$ and concentration $(\Phi)$ space are further discussed. The phase coexistence curve separates two regions: For all temperatures and compositions that lie outside the phase coexistence curve the two components mix homogeneously. The area inside the coexistence curve is called miscibility gap or phase coexistence region. For average compositions in the miscibility gap two different macroscopic phases emerge with compositions specified by the border of the phase coexistence region, see fig. 1.2.

The shape of the phase coexistence curve is connected to the free energy of the thermodynamic system. Since the entropic contribution favours mixing for higher temperatures the phase coexistence curve has the shape of a cap in a generic phase diagram. The miscibility gap becomes broader if temperature decreases. The maximum, where the two branches of the phase coexistence curve meet, is called the critical point. It is characterised by a critical temperature $T_{c}$ and a critical 
(a)

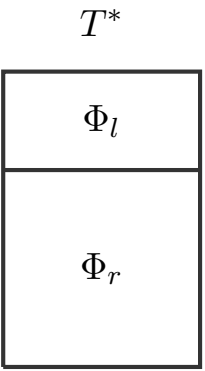

(b)

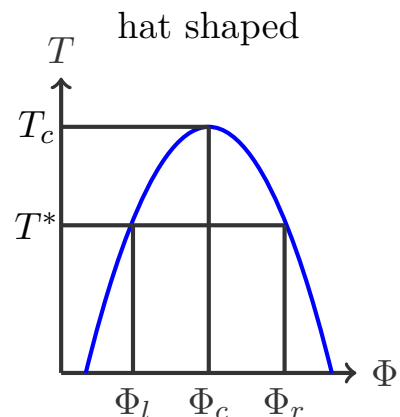

(c)

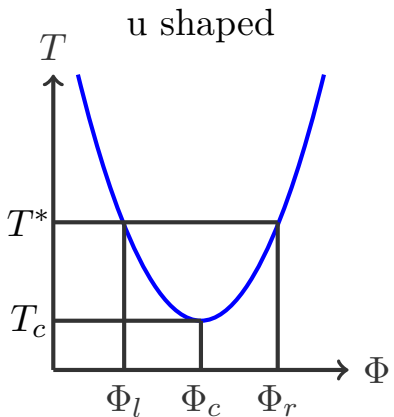

Figure 1.2: (a) For a temperature $T^{*}$ beyond the critical temperature the mixture separates into two macroscopic phase with the compositions $\Phi_{l}$ and $\Phi_{r}$. The mass density of the respective phases decides which phase is at the top. Panels (b) and (c) show a hat-shaped and a $\mathrm{u}$-shaped phase diagram, respectively. The critical point is described by the critical temperature $T_{c}$ and the critical composition $\Phi_{c}$. The mixtures phase separate upon cooling (b) or heating (c).

composition $\Phi_{c}$. Due to entropic contributions to the free energy, mixtures can show a u-shaped miscibility gap with a lower critical point, or even a loop-shaped miscibility gap (Yelash and Kraska, 1999; Walker and Vause, 1987). For the experiments on phase separation that will be reported later, I use isobutoxyethanol and water $(\mathrm{IBE}+\mathrm{W})$ mixtures. They have an u-shaped miscibility gap. This mixture is very convenient: it can be mixed at room temperature and phase separates under heating, see fig. 1.2(c). A similar mixture of butoxyethanol and water has been used for an experimental analog for mid-ocean ridge hydrothermal systems (Emmanuel and Berkowitz, 2006). A second mixture of methanol and hexane $(\mathrm{M}+\mathrm{H})$ is also used, it phase separates for cooling, see fig. 1.2(b).

A phase transition happens, if the temperature for a given average composition is changed across the phase coexistence curve. Doing the temperature variation quasi-statically, allows the determination of the transition temperature, and varying the compositions the phase coexistence curve can be measured.

For the dynamics of phase separation three different scenarios can be distinguished based on the applied temperature protocol (Vollmer, 2008). In fig. 1.3 I use the $\mathrm{u}$-shaped phase diagram with a lower critical point for visualization. Phase separation arises for a mixture with composition $\Phi$ when the temperature of the mixture is increased beyond the temperature $T_{b}(\Phi)$ marked by the binodal line. Let the system equilibrate at a temperature $T_{1}>T_{b}(\Phi)$ such that there are two macroscopic phases (see fig. 1.3). A further increase of temperature leads to a change in composition and therefore to a change of the volume fraction of the two macroscopic phases. Each temperature protocol corresponds to a ramp rate $\xi$, characterized by rate of 

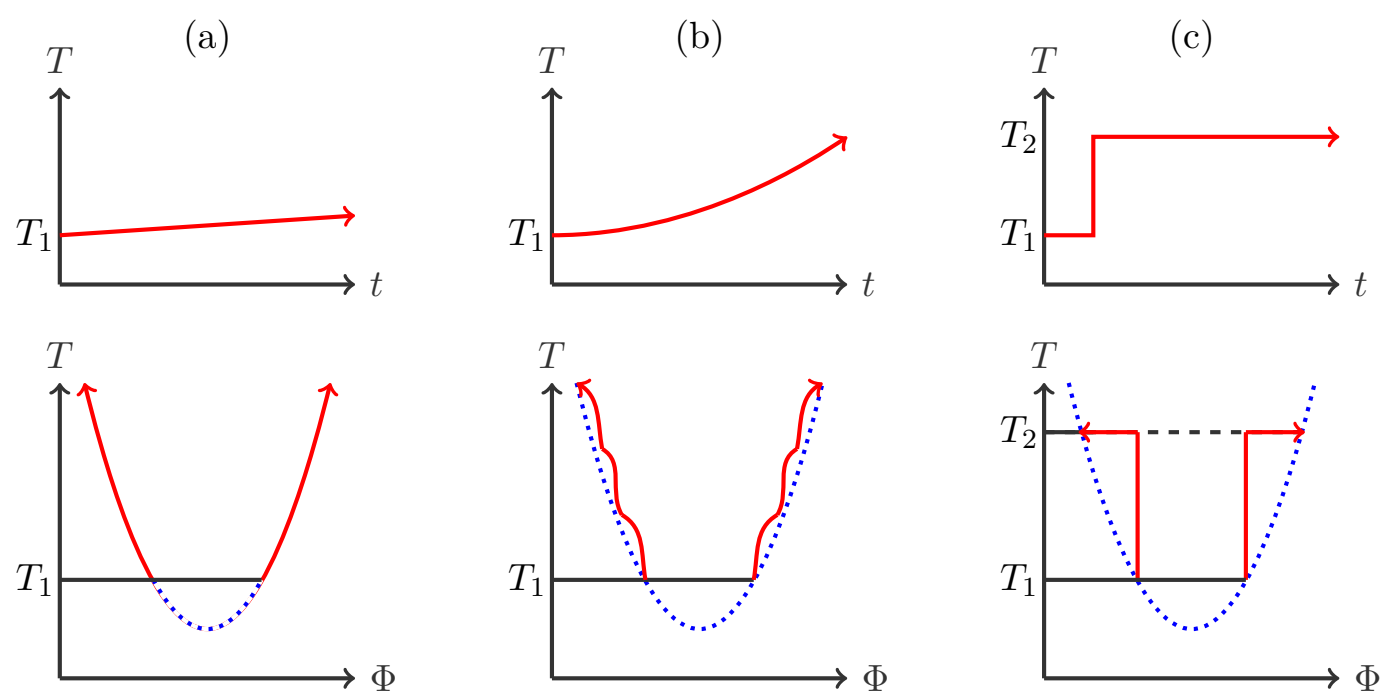

Figure 1.3: Phase separation scenarios for different temperature protocols $T(t)$, see upper part of the figure. (a) very slow temperature change, (b) intermediate change and (c) temperature quench. The lower part of the figure shows the phase coexistence curves (blue) in the phase diagrams and the corresponding evolution of the compositions (red).

the excess volume fraction that comes out of solution, if the mixture is equilibrated. Therefore I refer to the ramp rate $\xi$ also as the rate of volume fraction.

A quasi-static change in temperature is illustrated in fig. 1.3(a): in this setting the supersaturation relaxes by diffusion over the length scale $L$ of the system. Therefore, the red line in the lower panel of fig. 1.3(a) does not differ substantially from the phase coexistence curve. To observe this scenario the rate of the production of supersaturation, $\xi$, has to be much smaller than the rate of diffusive transport, characterised by its diffusion coefficient $D$. In our experiments we have have $L=$ $10^{-2} \mathrm{~m}$ and $D=10^{-10} \mathrm{~m}^{2} \mathrm{~s}^{-1}$. The upper bound for the ramp rate is (Vollmer, 2008)

$$
\xi \ll \frac{D}{L^{2}}=10^{-6} \mathrm{~s}^{-1}
$$

Typical ramp rates in our experiments are $\xi=10^{-5} \mathrm{~s}^{-1}$. Note that these are very slow temperature changes that are challenging to realize in a laboratory setting. However, in order to achieve quasi-static temperature changes even smaller rates are required.

The other extreme scenario is a sudden change of temperature, see fig. 1.3(c). Here the change of temperature is sufficiently fast to neglect changes in composition, during the experimental realization of the jump. This quench is followed by relaxation at 
constant temperature. Depending on the temperature change nucleation or spinodal decomposition is observed along the horizontal lines (Bray, 1994). This will relax the composition of the coexisting domaines to the respective equilibrium values of the binodal line. In either case this rapid relaxation is followed by coarsening of the domain structure. For many years phase separation was studied for isothermal conditions by these quench experiments.

Episodic precipitation arises in a scenario that lies between the very slow temperature change and the quench. It is observed for temperature changes that exceed the bound $\xi \gtrsim D / L^{2}$ provided in eq. (1.1). Fixing the ramp rate $\xi$ leads to well defined experimental conditions in terms of driven phase separation. This is done by adjusting the temperature protocol to the slope of the phase coexistence curve, keeping the change in composition constant. The slope of the phase coexistence curve increases for increasing temperature, thus also the slope of the temperature ramp in fig. 1.3(b) increases. The full details will be provided in section 2.3. As illustrated in fig. 1.3(b) the compositions evolve in a step like manner for such continuous driving. The composition stays the same for a while, when the temperature increases. As a consequence supersaturation builds up and eventually droplets will nucleate. Subsequently, on the length scale of droplet distances the supersaturation is relaxed by diffusion, and the composition moves back to the phase coexistence curve. Removing of droplets by coalescence and sedimentation leads to a reset of the system. Supersaturation will build up again. This leads to an oscillatory response of continuously driven phase separation.

In fig. 1.4 this behaviour is demonstrated for the phase separation of the mixture of IBE $+W$. The mixture forms two layers: less dense IBE-rich phase floating over the layer of the W-rich phase, see snapshots in fig. 1.4(a)-(f). In response to the temperature ramp both layers show an alternating variation in turbidity, fig. 1.4(a)-(f). Representing this evolution in a space-time plot, fig. 1.4(g), illustrates a variation of turbidity with a period $\Delta t_{i}$ between the $i^{\text {th }}$ and $(i+1)^{\text {st }}$ precipitation event.

The accompanying periodic alternation in the turbidity and the particle-size distribution are characteristics of episodic precipitation. The effect is robust. Episodic response has been observed in the particle-size distribution (Lapp et al., 2012) and in calorimetric data (Vollmer et al., 1997; Vollmer and Vollmer, 1999; Auernhammer et al., 2005; Mirzaev et al., 2010) in a vast range of binary mixtures (Vollmer et al., 1997; Auernhammer et al., 2005; Mirzaev et al., 2010; Lapp et al., 2012), including olive oil and methylated spirit (Vollmer et al., 2007). It arises in the upper as well as in the lower layer of the mixtures.

Vollmer et al. (2007) suggested a minimal model for the oscillation cycle. Benczik and Vollmer (2010) considered a reactive flow model to investigate the episodic precipitation in binary fluid demixing. Both models do not provide quantitative predictions of the time scale of the period $\Delta t$. In the present thesis such a prediction will be the topic of chapter 5 . 


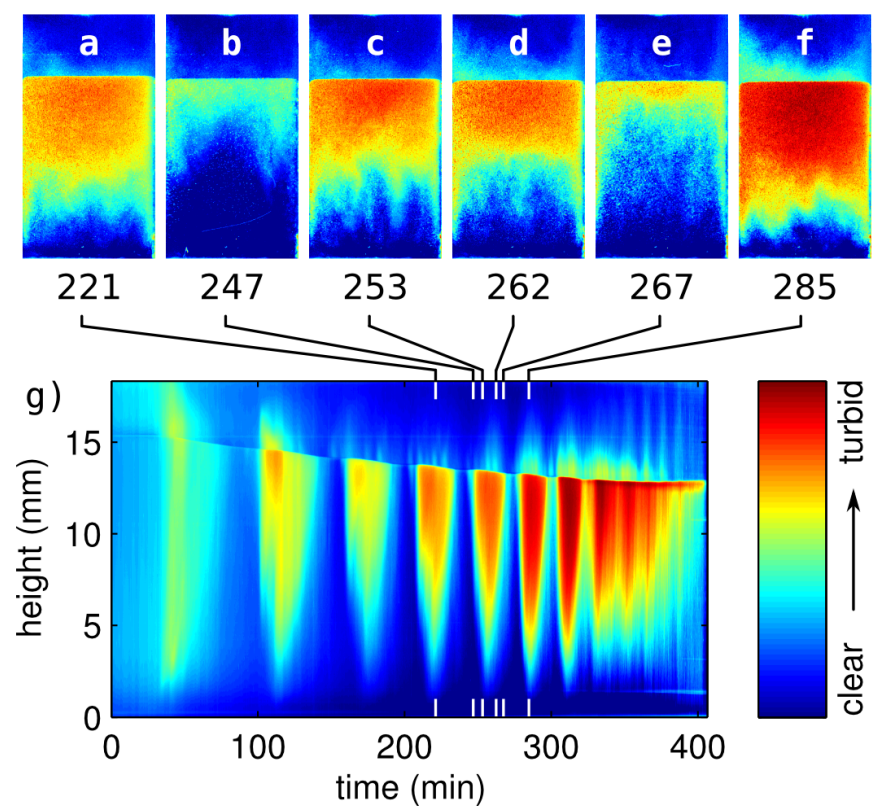

Figure 1.4: Episodic precipitation in binary mixtures. Panels (a)-(f) show false-colour plots of the turbidity distribution in snap shots of the phase separation of an isobutoxyethanol/water mixture subjected to a ramp rate, $\xi=2.5 \times 10^{-5} \mathrm{~s}^{-1}$. Averaging in horizontal direction and arranging the resulting vertical turbidity profiles next to each other produces a space-time plot of the time evolution of the turbidity, panel $(\mathrm{g})$. The length scale is provided on its ordinate axis, and the scales in the pictures (a)-(f) can be inferred by noticing that panel $(\mathrm{g})$ shows the full height of the samples. In chapter 2 I provide full details on the experimental setup and method.

\subsection{Ostwald ripening and Lifshitz-Slezov-Wagner theory}

The growth rate of a droplet depends on the supersaturation in the surrounding of the droplet. In an assembly of droplets they influence each other. Ostwald (1900) first described the evolution of such an assembly at constant temperature: The mean radius grows and the number of aggregates decreases, whereas the total volume of aggregates is constant. The redistribution of aggregate volume is driven by minimizing the interfacial energy of the system.

This dynamics involves a delicate balance of the evaporation of small aggregates, and redistributing their volume to achieve further growth of large aggregates. The radius dependent growth rate of the aggregates can be found by dimensional analysis. The change of aggregate volume is proportional to the diffusion constant, $D$, characterising the transport of material. By dimensional arguments it is also proportional to a length scale. This length scale should involve the radius of the droplets. Moreover, 
there must be a critical radius, $R_{c}$, such that larger droplets grow, and smaller droplets shrink. This suggests the following evolution of an aggregate with radius $R_{i}$

$$
R_{i}^{2} \dot{R}_{i} \sim D\left(R_{i}-R_{c}\right)=R_{c} D\left(\frac{R_{i}}{R_{c}}-1\right) .
$$

Introducing a length scale $\sigma$, that accounts for dimensionless prefactor in eq. (1.2), one obtains for the radius growth rate

$$
\dot{R}_{i}=\frac{D \sigma}{R_{i}^{2}}\left(\frac{R_{i}}{R_{c}}-1\right)
$$

The length scale $\sigma$ depends on the material parameters, that set the material transport. It is denoted as the Kelvin length. As expected, $\sigma$ is proportional to the interfacial tension $\gamma$ which provides the energy penalty driving coarsening. A derivation of eq. (1.3) based on the explicit solution of the diffusion equation is given e.g. in Landau and Lifshitz (1983) and Bray (1994). It leads to eq. (1.3) with

$$
\sigma=\frac{2 \gamma V_{m} \Phi}{R T}
$$

The equation for the growth rate, eq. (1.3), together with the conservation of aggregate volume allows us to determine the value of the critical radius $R_{c}$. Since the overall volume

$$
V=\frac{4 \pi}{3} \sum_{i=1}^{N} R_{i}^{3}
$$

is conserved, we obtain

$$
0=\dot{V}=4 \pi \sum_{i=1}^{N} R_{i}^{2} \dot{R}_{i}=4 \pi D \sigma\left(\frac{N\langle R\rangle}{R_{c}}-N\right)
$$

where $\langle R\rangle=\frac{1}{N} \sum_{i}^{N} R_{i}$ is the average radius. Therefore, eq. (1.6) is fulfilled only for $R_{c}=\langle R\rangle$. Independently, Lifshitz and Slyozov (1961) and Wagner (1961) derived this relation as well as scaling laws for the decay of the number of aggregates, the resulting growth speed of the mean aggregate radius, and they determined the shape of the asymptotic size distribution. Their results are known as the LSW-theory of Ostwald ripening. Modern expositions derive their results from the point of view of dynamic scaling theory (Voorhees, 1985; Bray, 1994; Barenblatt, 2003), i.e. as a scaling solution of the continuity equation for the aggregate number density per radius $n(R, t)$

$$
\frac{\partial n}{\partial t}+\frac{\partial}{\partial R}(\dot{R} n)=0 .
$$


Here $n$ is a conserved quantity, because only evaporation at zero size (boundary term) and growth is considered, i.e. the aggregates do not coalesce.

\subsection{Ripening with material flux}

In the examples discussed in section 1.1 the aggregate assembly does not evolve at a constant aggregate volume fraction. Rather it increases due to driving of the phase separation. Here we consider the same equation for the growth rates as discussed for the LSW theory, eq. (1.3), but for $\dot{V}=\xi \mathcal{V}$, rather than $\dot{V}=0$. The ramp rate $\xi$ denotes the change in aggregate volume fraction and $\mathcal{V}$ the sample volume. This kind of conservation law gives

$$
\begin{aligned}
\xi \mathcal{V} & =\dot{V}=4 \pi \sum_{i=1}^{N} R_{i}^{2} \dot{R}_{i}=4 \pi D \sigma\left(\frac{N\langle R\rangle}{R_{c}}-N\right) \\
\Rightarrow k & :=\frac{\langle R\rangle}{R_{c}}=1+\frac{\xi \mathcal{V}}{4 \pi D \sigma N}=1+\frac{\xi}{4 \pi D \sigma n}
\end{aligned}
$$

with $n=N / \mathcal{V}$ being the number density of aggregates. The ratio $k$ of the average aggregate radius and the critical radius has also been identified by Clark et al. (2011) as the relevant parameter that governs the evolution of the aggregate size distribution with overall volume growth. When there is a net growth of overall volume, the control parameter $k$ is increased by the ratio of the growth rate $\xi$, and the diffusive relaxation of supersaturation $4 \pi D \sigma n$. Substituting the critical radius in the equation of the growth rate, eq. (1.3), by $R_{c}=\langle R\rangle / k$, (see eq. (1.9)) we find the following set of equations for the evolution of the aggregate radius $R_{i}$

$$
\dot{R}_{i}=\frac{\sigma D}{R_{i}^{2}}\left(k \frac{R_{i}}{\langle R\rangle}-1\right) .
$$

The growth of the aggregate radii, $R_{i}$, is coupled in a mean-field way via the dependence of the equations on the average aggregate radius $\langle R\rangle$, and via $k$ also to the number density, $n$, of the aggregates (cf. eq. (1.9)).

Equation (1.10) combines the growth law of aggregates for $k=1$, i.e. the starting point of the LSW theory, and for $k>1$, i.e. the case of ripening with overall volume growth, that leads to episodic precipitation, emerging for larger values of $k$. In chapter 4 we will see that qualitative changes emerge in the evolution of the size distribution, when increasing $k$ from $k \gtrsim 1$ towards larger values.

Early successes in the theoretical modelling of aggregate-size distributions focused on describing the diffusive transport of material to the aggregates (LaMer and Dinegar, 1950). In many applications the volume fraction of the aggregates grows during the ripening - either due to feeding by a chemical reaction, or because temperature or 

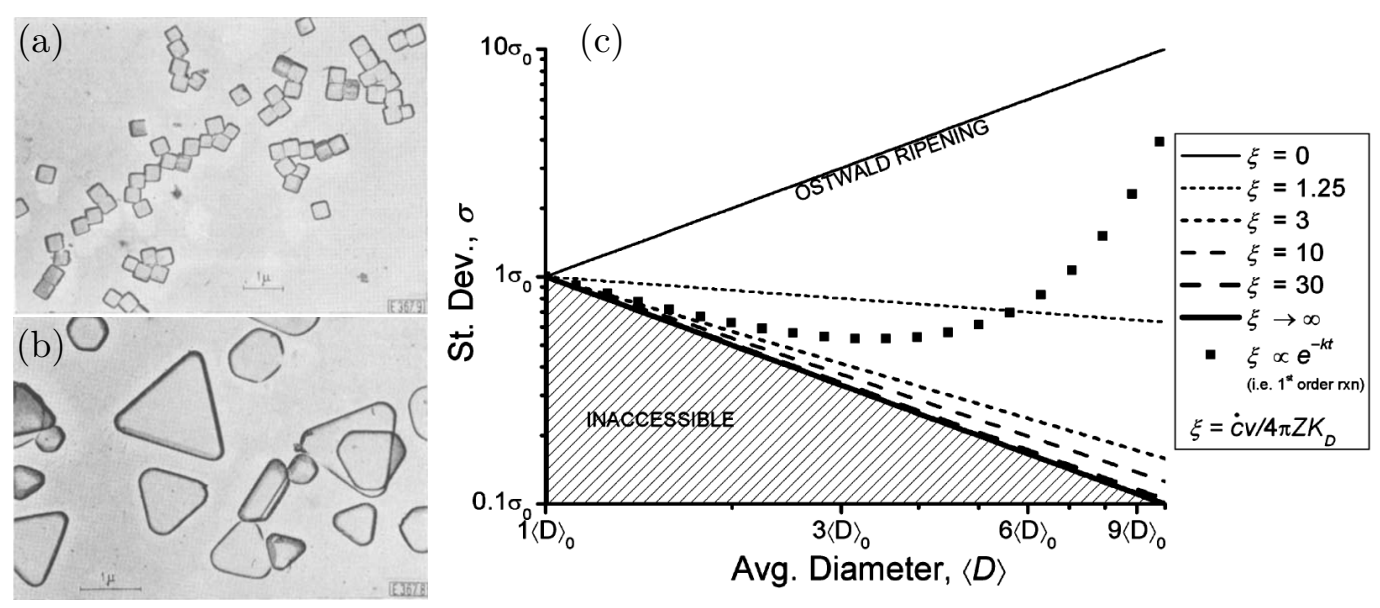

Figure 1.5: Size focussing in colloid synthesis. (a) Monodisperse silver particle for photographic films obtained with a material flux into thy system. (b) Silver particles grown without a sustained material flux, given for comparison. Reprinted from Klein and Moisar (1963). (c) Relation of the width of the distribution characterised by the standard deviation $\sigma$ and the diameter of the aggregate $\langle D\rangle$ for different values of $k$. The value of $\xi$ in the figure corresponds to $k-1=\xi /(4 \pi D \sigma n)$ in our notation, i.e. it agrees with our notation of $\xi$ up to non-dimesionalization. Reprinted from Clark et al. (2011).

pressure changes lead to a change of the equilibrium volume fraction of the aggregates. Reiss (1951) pointed out that the resulting sustained growth of the volume fraction of the aggregates can lead to focusing of the aggregate size distribution (see Kwon and Hyeon, 2011; Clark et al., 2011; Sowers et al., 2013, for recent discussions). An early application of the focussing of the size distribution was the synthesis of monodisperse silver particles for photographic films, see fig. 1.5(a,b).

Recently Clark et al. (2011) obtained a relation between the width of the size distribution and the mean diameter of the aggregates, depending on the value of control parameter $\xi /(4 \pi D \sigma n)=k-1$, see fig. 1.5(c). They based their analysis on a projection of the dynamics to Gaussian size distributions: The width and the mean diameter of the aggregates are allowed to evolve whereas the shape of the distribution is fixed to be a Gaussian. For $\xi=0$ the distribution becomes broader, as observed in Ostwald ripening, whereas size focussing is observed for $\xi /(4 \pi D \sigma n) \gtrsim 1$ i.e. for $k \gtrsim 2$.

\subsection{Collisions and sedimentation}

All phenomena that show episodic precipitation must have a mechanism that enhances aggregate growth in a such way that precipitation can occur in a finite time. In the 

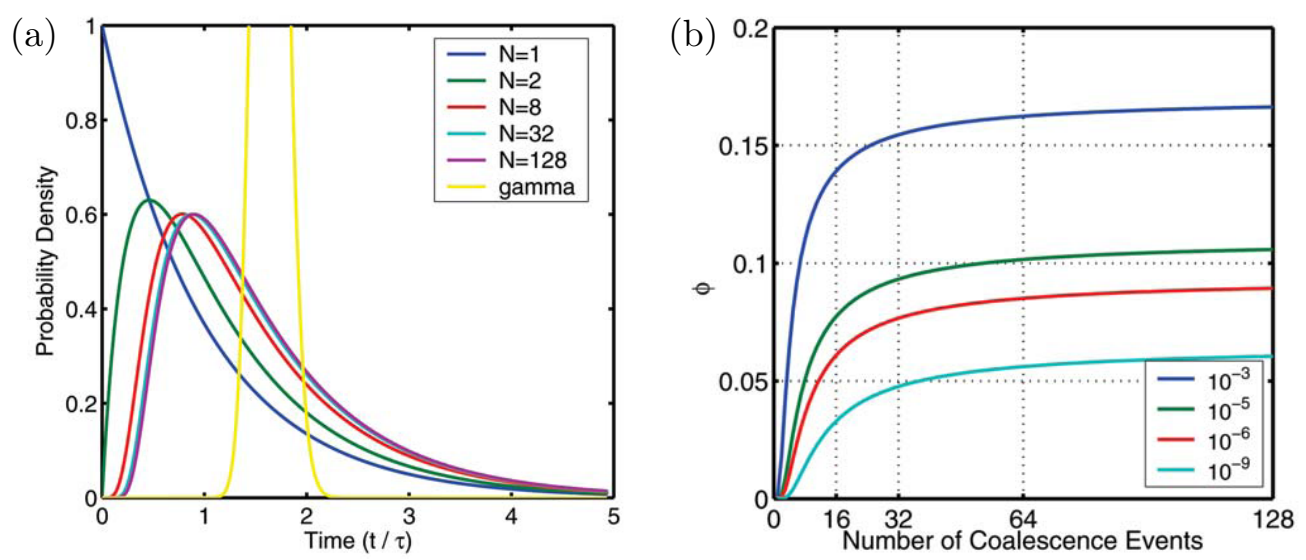

Figure 1.6: (a) Generalized Erlang distributions show the distribution of times for $N$ coalescence events, where $\tau$ is the average waiting time for the first coalescence. (b) Speedup factor $\phi$ as a function of the number of coalescence events $N$ for different factions of droplets that collect the smaller droplets, as indicated by the legend. Reprinted from Kostinski and Shaw (2005).

following I concentrate on the initiation of warm rain. Here ideas and mechanism were developed in the cloud physics community that I will adapt to the modelling of my experiments in the following chapters.

Consider a cloud consisting of $10 \mu \mathrm{m}$ cloud droplets (see fig. 1.1 at initial times). A droplet growing to the size of $1 \mathrm{~mm}$ needs to coalescence with one million other $10 \mu \mathrm{m}$-sized droplets. In practice a fraction of $10^{-6}$ of the largest droplets in the tail of the distribution take over the distribution by collecting all the volume distributed in the smaller droplets. To discuss this kind of droplet growth I follow Kostinski and Shaw (2005). Rather than applying Smoluchowski integro-differential equations they gained insight in this process by adopting a collector drop scenario of one drop falling through a cloud of identical smaller droplets - a scenario initially introduced by Telford (1955).

Starting at $10 \mu \mathrm{m}$ a randomly selected "fortunate" droplet has its first coalescence much earlier than the average time for the first coalescence $\tau$. Since it is larger now, it is more likely than others to encounter another coalescence. Indeed, the time between two coalescence events is proportional to the cross section of the droplet, its sedimentation speed and the collection efficiency. Kostinski and Shaw (2005) assume the following relation of the time $\tau$ between the $n$th and the $(n+1)$ th coalescence event on the volume of the droplet $v=n \cdot v_{0}$

$$
\tau_{n} \sim\left(n \cdot v_{0}\right)^{-2}
$$


where $v_{0}$ denotes the volume of one of the small droplets, that are collected. The time between the coalescence events decays rapidly. Thus only the first terms really contribute to the time of $N$ coalescence events. The distribution of waiting times is virtually the same for all $N>128$, fig. 1.6(a). The curve for the time to the first coalescence is an exponential distribution with a mean time $\tau$. In the framework of inhomogeneous Poisson processes the distribution of times for $N$ coalescence events is a convolution of the exponential distributions with decaying mean times, yielding the generalized Erlang distributions, shown in fig. 1.6(a). These distributions rapidly converge - there is only a small difference between the distributions of $N=8$ and $N=128$, and almost no difference between the distributions corresponding to $N=32$ and $N=128$. Hence, if a droplet manage to collect a few other droplets, the process of growth is started and the time for the approximately other 100 coalescence events is not much longer. Once a droplet has encountered 10 collisions, another 100 will follow soon, i.e. on a time scale much smaller than $\tau$, and at that point the collision frequency diverges. The resulting finite time runaway leads to an efficient and fast initiation of rain.

In fig. 1.6(b) the speedup in growth rate is shown as a function of the number of coalescence events $N$. It is given for different fractions of droplets, that are able to collect the volume of a cloud. The curves for $10^{-5}$ and $10^{-6}$ droplet fraction are in agreement with droplet concentrations in warm rain initiation (Kostinski and Shaw, 2005). In this case, the growth rate is 10 times faster than the mean growth rate.

\subsection{Outline}

The present thesis has four parts. Following the introduction (chapter 1) I describe the adopted tools and methods (chapters 2 and 3). Subsequently, different aspects of episodic precipitation in binary mixtures are addressed in chapters 4 to 6 . These case studies are complementary and build partially on their mutual insights. Finally, the results are combined and interpreted in the context of their application to other systems (chapters 7 and 8).

In chapter 2 I provide the experimental techniques and methods used throughout the thesis. From the beginning I could use an experimental setup designed for measuring the evolution of the size distribution. It was developed by Tobias Lapp during his PhD thesis (Lapp, 2011) and by myself during my diploma thesis (Rohloff, 2011). The setup has been described in detail in Lapp (2011), and in the joint publication Lapp et al. (2012). Here, I focus therefore on the description of an extension of the setup that allows us now to measure the turbidity, and on my efforts to characterize the temperature ramps in much more detail. 
In chapter 3 I describe an efficient algorithm to integrate an assembly of aggregates, that is used in chapter 4 to study the impact of overall volume growth on Ostwald ripening. This exposition follows our publication Vollmer et al. (2014).

Chapter 4 has two parts. The first one deals with the size distributions of Brownian aggregates with an overall volume growth. The theory for large $k$ values and the results for the uniform initial distribution has also been published in Vollmer et al. (2014). In addition the evolution of a bell-shaped and a bimodal distribution is shown to study the impact of the initial distribution.

The second part addresses the impact of sedimentation on the droplet size distributions. Here I provide a consistent interpretation of the data and I identify the cause of the emerging bimodal distribution as sedimentation. These results of the second part have been obtained in collaboration with Marcel Ernst, who worked together with me during his Bachelor thesis (Ernst, 2014).

Chapter 5 is concerned with the time scale of the oscillation period emerging in episodic precipitation. First, I provide the data of oscillations periods in IBE $+\mathrm{W}$ mixture that has been obtained in collaboration with Tobias Lapp (Lapp, 2011), and in $\mathrm{M}+\mathrm{H}$ mixtures from turbidity measurements. Subsequently, I address the influence of flow on the oscillation period. This work has been done in collaboration with Julian Vogel, who joined my project for a Bachelor thesis (Vogel, 2013). The model of the precipitation cycle builds upon the formulation of Lapp (2011). It was inspired by Michael Wilkinson's idea to use Ostwald ripening as the growth mechanism for small droplets (Wilkinson, 2014). However, the overall volume growth leads us to adopt the theory of droplet growth for large $k$ values that is described in chapter 4 . This leads to a quantitative agreement with our experimental data (Rohloff et al., 2015), whereas Ostwald ripening fails (Rohloff et al., 2014).

In chapter 6 I explore the impact of periodic driving. Here Marcel Ernst provided numerical data and Julian Vogel lied the basis with his experimental observations. Julian was assisted by Darcy Jacobson, a RISE summer student, whom I have supervised. The model for the precipitation cycle described in chapter 5 for constant driving is extend for a periodic driving. The analytic approximation of the theory as well as numerical data predict regions of synchronisation, that are also observed experimentally. Additionally the impact of high frequency driving is investigated.

In chapter 7 I come back to the examples of rain formation, colloid synthesis and geysers, that were used to motivate my work in section 1.1. I discuss them here based the insights and results of the previous chapters.

Finally, I conclude the thesis in chapter 8. 



\section{Experimental techniques and methods}

In this chapter I describe the experimental setup and the procedures of data analysis used in this thesis. Starting point is the description of the experimental setup for measuring turbidity of the sample and the size distributions during phase separation. Then the binary mixtures are characterised by their phase diagrams, which are subsequently used to calculate temperature ramps for driving the phase separation in a controlled way. Finally the optical setups and the data analysis is described for measuring the turbidity and the size distributions, respectively.

\subsection{Experimental setup for investigating binary fluid demixing}

The experiments on demixing reported in this thesis focus on the oscillatory behaviour, which occurs for slow continuous changes of temperature. The oscillatory behaviour manifests itself in an oscillating turbidity of the sample which can even be observed by eye, as well as oscillations in the size distribution. The presence of many droplets as a result of the demixing causes the scattering of light and the sample will appear turbid and give bright images. I will therefore refer to the turbidity as the gray value of the images. For measuring the period of the oscillations it is sufficient to record the turbidity, which is easily monitored for the whole sample and straight forward to extract from the images. On the other hand, I record images with a sufficient magnification to identify individual droplets. The detection of droplet sizes allows to obtain the time evolution of the size distribution of droplets. Both techniques differ mainly in the setup of the optics for the illumination and the image acquisition. Therefore, I will discuss first the general setup, that both techniques have in common. Subsequently, the optical setups and the data processing are discussed separately in sections 2.4 and 2.5 .

For the investigation of the demixing a few millilitre of the fluid mixture is placed in a fluorescence cell 117.100F-QS made by Hellma GmbH (see fig. 2.1 (1) and (1a)). The cell is immersed in a thermostated water bath in order to drive the demixing by changing the temperature. The temperature control of the water bath has two components: an immersion cooler Haake EK20 (5), which cools with a constant power and a computer controlled thermostat Huber CC-E (4), which heats the water to a preset temperature. Additionally the temperature near the sample is measured 


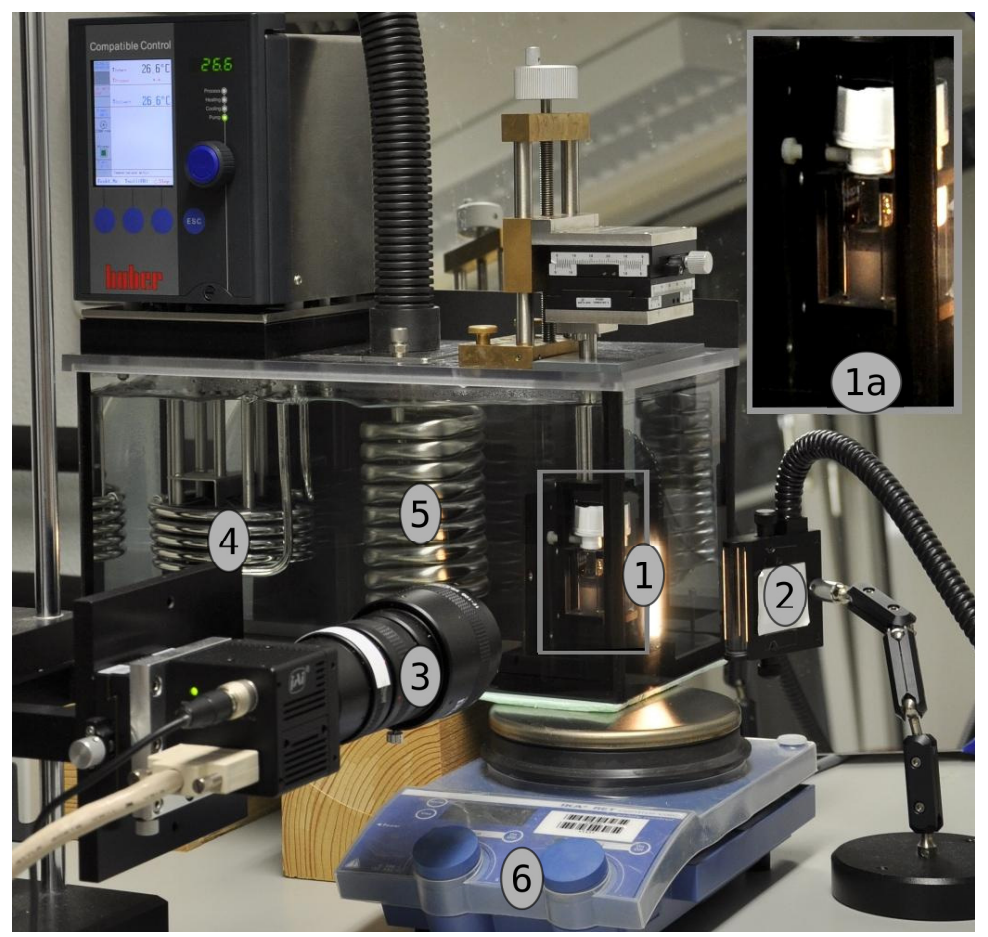

Figure 2.1: Experimental setup. Sample (1), thermostat (4) and cooler (5) are immersed into the water bath. Illumination light source (2) and camera (3) are placed at two sides of the water bath, and the magnetic stirrer (6) below.

with a PT100 sensor. The temperature is controlled with an accuracy of $15 \mathrm{mK}$. Images are taken with a BM-500CL monochrome progressive scan CCD camera (3). The sample is illuminated with a light source (2), which depends on the respective setup and will be described later. A magnetic stirrer (6) enables homogenisation of the fluid mixture between two experimental runs. It also allows us to study the influence of an external flow caused by the stirring of the magnetic stirring bar, see section 5.1.2.

The whole setup, the temperature as well as the camera and the magnetic stirrer, are controlled by the computer via a LabVIEW program.

In this form I found the setup ready for experiments at the beginning of my $\mathrm{PhD}$. However, I redesigned the thermostating unit (thermostat and cooler) to make it proof against freezing with a temperature dependent switch to turn off the cooling automatically, if the temperature of the water bath drops below a threshold of $5^{\circ} \mathrm{C}$. The LabVIEW program to run the experiments has typically three steps:

- Homogenisation: The binary fluid is homogenised by shaking by hand outside the water bath or by the magnetic stirrer at a temperature sufficiently far in 
the one phase region of the phase diagram.

- Relaxation: The temperature is changed to $T_{0}$ across the phase coexistence curve into the two-phase region of the phase diagram. This temperature is held constant for about $180 \mathrm{~min}$. This time is sufficient for forming two layers with equilibrium compositions.

- Temperature ramp: Starting from the equilibrated sample at $T_{0}$ the temperature of the water bath is changed according to a preset temperature ramp. The ramp can be provided either in dimensionless times or in units of seconds. The temperature of the thermostat is set every second, images are recorded at a specified frame rate in the range of $1 \mathrm{~Hz}$ to $0.05 \mathrm{~Hz}$.

A second mode of operation of the LabVIEW program is the determination of the transition temperature of binary mixtures. This has been used extensively to determine the phase diagram of isobutoxyethanol and water (Rohloff, 2011). The basic idea is to change the temperature across the phase coexistence curve and monitor the turbidity(Dean et al., 2010). An abrupt change in turbidity indicates the phase transition.

\subsection{Phase diagrams of binary fluid mixtures}

In this thesis I report on experiments with two mixtures: isobutoxyethanol/water $(\mathrm{IBE}+\mathrm{W})$ and methanol/hexane $(\mathrm{M}+\mathrm{H})$. Both mixtures are characterized in the following by their phase diagrams fig. 2.2.

Mixtures of $\mathrm{M}+\mathrm{H}$ are one of the classical model systems of binary phase separation (Huang et al., 1974; Abbas et al., 1997; Sam et al., 2011). The two fluids are fully miscible above $34.45^{\circ} \mathrm{C}$. Mixtures of IBE $+\mathrm{W}$ are an experimentally-friendly system, that phase separates upon heating. The mixture can be homogenised at room temperature. Mixtures of water and butoxyethanol have become popular as an experimentally-friendly system that phase separates upon heating (see e.g. Emmanuel and Berkowitz (2006)). For our present purposes IBE and water, is even preferential since the critical point of the mixtures, $T_{c}=25.61^{\circ} \mathrm{C}$, lies more than $10^{\circ} \mathrm{C}$ below the one of the butoxyethanol mixture. This further enhances the range of experimentally accessible temperatures (that must always lie well below the boiling point of water). In Rohloff (2011) and Lapp (2011) polynomial fits are used to fit the experimental data for the phase coexistence curve. I use a forth or sixth order polynomial to fit each branch of the coexistence curve,

$$
T(\Phi)=T_{c}+\sum_{n=2}^{6} a_{n}\left(\Phi-\Phi_{c}\right)^{n} .
$$


(a)

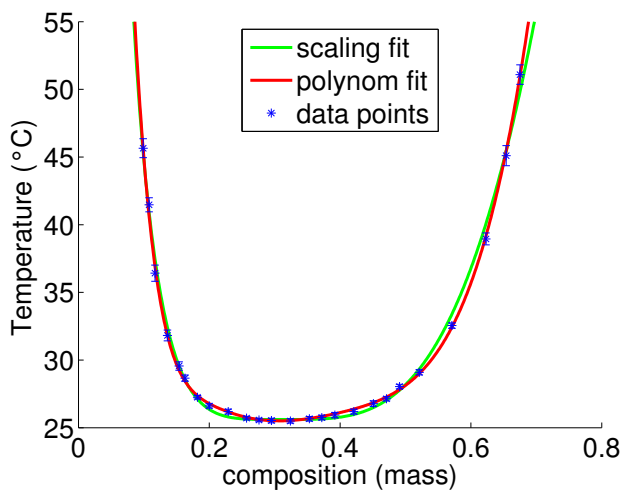

(b)

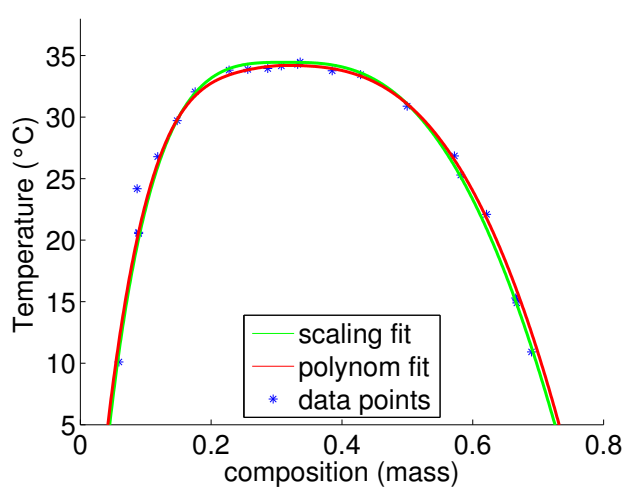

Figure 2.2: Phase diagrams of (a) $\mathrm{IBE}+\mathrm{W}$ and (b) $\mathrm{M}+\mathrm{H}$. The respective concentrations are given in mass fraction of IBE and M. The data points are shown by blue symbols and the polynomial (eq. (2.1)) and scaling fits (eq. (2.2)) of the phase coexistence curve by red and green solid lines, respectively. The data points of the IBE $+\mathrm{W}$ mixture denote own measurements and the data of $\mathrm{M}+\mathrm{H}$ has been adapted from Auernhammer et al. (2005).

The first order term is set to zero to have a zero slope at the critical point, and $T_{c}$ and $\Phi_{c}$ denote the values of the temperature and the composition at the critical point. The fit parameters for $\mathrm{IBE}+\mathrm{W}$ and $\mathrm{M}+\mathrm{H}$ are given in table 2.1. The critical compositions in mass fractions are $\Phi_{c}=0.310$ for IBE $+\mathrm{W}$ and $\Phi_{c}=0.313$ for $\mathrm{M}+\mathrm{H}$. In a recent paper Wilkinson (2014) suggested that critical scaling exponents might be relevant to describe the data of the oscillation periods (chapter 5). To explore this connection Rohloff et al. (2015) adopted a more profound function for the phase coexistence curve. For this purpose the procedure of Aizpiri et al. (1990) is used to

\begin{tabular}{ccccc}
\hline & \multicolumn{2}{c}{ IBE+W } & \multicolumn{2}{c}{$\mathrm{M}+\mathrm{H}$} \\
$\Phi_{c}$ & \multicolumn{2}{c}{0.310} & \multicolumn{2}{c}{0.313} \\
$T_{c}\left({ }^{\circ} C\right)$ & \multicolumn{2}{c}{25.51} & \multicolumn{2}{c}{34.20} \\
& left & right & left & right \\
\hline$a_{2}$ & 51.2 & 122.2 & -159.7 & -23.0 \\
$a_{3}$ & 979.8 & -750.7 & -1339 & -373.9 \\
$a_{4}$ & 995.3 & 2574.2 & -8155 & 73.7 \\
$a_{5}$ & 120779 & - & - & - \\
$a_{6}$ & 647056 & - & - & - \\
\hline
\end{tabular}

Table 2.1: Parameters of the polynomial fit of the phase diagram of IBE $+\mathrm{W}$ and $\mathrm{M}+\mathrm{H}$ according to eq. (2.1). 


\begin{tabular}{lcc}
\hline & IBE $+\mathrm{W}$ & $\mathrm{M}+\mathrm{H}$ \\
$\beta$ & 0.25 & 0.325 \\
$T_{c}(\mathrm{~K})$ & $298.76 \pm 0.12$ & $307.88 \pm 0.15$ \\
$\Phi_{c}$ & $0.3093 \pm 0.0032$ & $0.3143 \pm 0.0008$ \\
$B$ & $0.547 \pm 0.002$ & $0.726 \pm 0.002$ \\
$D$ & $0.26 \pm 0.015$ & $0.323 \pm 0.005$ \\
\hline
\end{tabular}

Table 2.2: Fit parameters of the coexistence curve for $\mathrm{IBE}+\mathrm{W}$ and $\mathrm{M}+\mathrm{H}$ according to eq. (2.2).

express the left and right branch of the coexistence curve by

$$
\Phi_{r / l}=\Phi_{c} \pm B \theta^{\beta}+D \theta^{2 \beta} \quad \text { with } \quad \theta=\left|1-\frac{T}{T_{c}}\right|
$$

in terms of the reduced temperature $\theta$. Here, again the critical point is at the temperature $T_{c}$ and the concentration $\Phi_{c}$. However, now the shape of the phase boundary is described by the nontrivial scaling exponent $\beta$. Its universal value at the critical point $\beta=0.325$ has been calculated by renormalization group theory (Stanley, 1971).

The fit parameters for the two mixtures are collected in table 2.2. For $\mathrm{M}+\mathrm{H}$ eq. (2.2) provides an excellent fit for $\beta=0.325$. In contrast for $\mathrm{IBE}+\mathrm{W}$ it is not possible to get a reasonable fit for $\beta=0.325$. It is reported (Nakata et al., 1982) that the universal scaling exponent only applies for $\theta<10^{-3}$ which is below the temperature range relevant for our experiments. Therefore, I choose $\beta=0.25$ to gain a faithful description.

\subsection{Driving the demixing by temperature ramps}

The demixing of the binary liquids in my experiments is driven by a continuous change of the temperature. A temperature change involves a change in the equilibrium composition, which eventually results in a change of the volume fractions of droplets and bulk. In the present section I discuss how the form of the phase coexistence curve is connected to the driving $\xi$.

\subsubsection{Calculating $\xi$ from the form of the phase-coexistence curve}

Earlier work on the thermodynamic driving of the demixing of binary liquids concentrated more on a theoretical view coming from a nonlinear diffusion equation (Cates et al., 2003; Auernhammer et al., 2005). Here I will take an approach motivated from the experimental side which allows me to connect the driving force directly to 
the production of droplet volume fraction as a result of the demixing process ${ }^{1}$. The connection of the driving to the change in droplet volume fraction was previously specified in Rohloff (2011) and Lapp (2011). However, here I revisit the derivation on a more profound basis.

The derivation of the ramp rate $\xi$ starts with considering a volume containing droplets with a volume fraction $v_{d}$ and a composition $\Phi_{d}$ surrounded by fluid of the bulk composition $\Phi_{b}$. The average composition $\Phi$ in this volume is

$$
\Phi=v_{d} \Phi_{d}+\left(1-v_{d}\right) \Phi_{b} .
$$

Changing the temperature will change the equilibrium compositions of droplets and bulk but the average composition will stay the same, so that

$$
0=\dot{\Phi}=v_{d} \dot{\Phi}_{d}+\Phi_{d} \dot{v}_{d}+\left(1-v_{d}\right) \dot{\Phi}_{b}-\Phi_{b} \dot{v}_{d} .
$$

Introducing the following definitions

$$
\begin{aligned}
\zeta_{0} & =\Phi_{0}^{-1} \frac{\mathrm{d} \bar{\Phi}}{\mathrm{d} t} \\
\xi_{0} & =\Phi_{0}^{-1} \frac{\mathrm{d} \Phi_{0}}{\mathrm{~d} t} \\
\varphi & =\frac{\Phi-\bar{\Phi}}{\Phi_{0}} \\
\text { where } \quad \bar{\Phi} & =\frac{1}{2}\left(\Phi_{b}+\Phi_{d}\right), \\
\Phi_{0} & =\frac{1}{2}\left(\Phi_{b}-\Phi_{d}\right),
\end{aligned}
$$

eq. (2.4) rearranges to:

$$
\dot{v}_{d}=\frac{1}{2}\left(\zeta_{0}+\xi_{0} \varphi\right) .
$$

This means that the change in droplet volume fraction due to a change in temperature has two contributions. The first one $\zeta_{0}$ comes from the asymmetry of the phase diagram, i.e. the change of the midpoints $\bar{\Phi}$ of the miscibility gap, whereas the second one $\xi_{0}$ comes from the change of the width $\Phi_{0}$ of the miscibility gap. $\varphi$ is the reduced average composition, it is 1 for $\Phi=\Phi_{b}$ and smaller for values inside the

\footnotetext{
${ }^{1}$ For all practical purposes the demixing process starts with the creation of supersaturation, which then relaxes after nucleation of droplets by the diffusion of the minority phase to the droplet, forming a volume fraction in its literal sense. In this derivation all volume which is off the equilibrium volume distribution will be denoted as volume fraction of droplets, no matter if it is still dissolved as supersaturation or already belonging to droplets.
} 


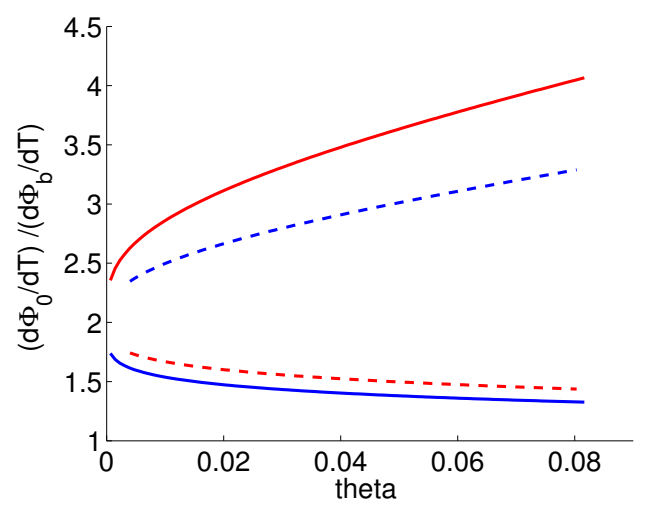

Figure 2.3: $\dot{\Phi}_{0} / \dot{\Phi}_{b}$ from eq. (2.7) for IBE $+\mathrm{W}$ (solid lines) and $\mathrm{M}+\mathrm{H}$ (dashed lines) and for the driving held constant in the upper layer (red) and the lower layer (blue) of the mixture.

miscibility gap.

The local bulk composition is then characterised by the local reduced composition which is now space and time dependent $\varphi(x, t)$. Its evolution is described by a diffusion equation which contains a source term of $2 \dot{v}_{d}$ (Auernhammer et al., 2005; Vollmer et al., 2007). From the view point of the transport equation it is desirable to fix the source term $\dot{v}_{d}$ rather than fixing $\xi_{0}$ or $\zeta_{0}$ as done in Auernhammer et al. (2005).

In order to gain an expression for the source term, eq. (2.6), depending on the compositions $\Phi_{d}$ and $\Phi_{b}$ the definitions in eq. (2.5) are inserted back into eq. (2.6). This provides

$$
\dot{v}_{d}=\frac{1}{2 \Phi_{0}(T)} \frac{\mathrm{d} \Phi_{b}}{\mathrm{~d} t}-\frac{v_{d}}{\Phi_{0}} \frac{\mathrm{d} \Phi_{0}}{\mathrm{~d} t}=\frac{\dot{\Phi}_{b}}{2 \Phi_{0}}\left(1-2 v_{d} \frac{\dot{\Phi}_{0}}{\dot{\Phi}_{b}}\right) \approx \frac{1}{2 \Phi_{0}(T)} \frac{\mathrm{d} \Phi_{b}}{\mathrm{~d} T} \frac{\mathrm{d} T}{\mathrm{~d} t} .
$$

In the last step I used the fact that in our experiments the droplet volume fraction $v_{d}$ is small, in the order of a few percent and the ratio $\dot{\Phi}_{0} / \dot{\Phi}_{b}$ is of order 1 , see fig. 2.3. In order to denote the driving which is described by $\dot{v}_{d}$ also with $\xi$ to use the same terminology as in Cates et al. (2003); Vollmer and Vollmer (1999); Auernhammer et al. (2005); Vollmer et al. (2007); Lapp et al. (2012) I identify the change in droplet 
volume fraction with the driving ${ }^{2} \xi$

$$
\xi=\frac{1}{2 \Phi_{0}(T)} \frac{\mathrm{d} \Phi_{b}}{\mathrm{~d} T} \frac{\mathrm{d} T}{\mathrm{~d} t} .
$$

If it is necessary to specify the bulk phase to which the driving $\xi$ refers, there will be an index $\xi_{l}$ or $\xi_{r}$ referring to the left or right branch of the coexistence curve of the phase diagram describing the composition of the respective bulk phase. Otherwise the index will be dropped. Note that $\xi_{l}+\xi_{r}=\xi_{0}$, which was kept constant in former work on demixing (Auernhammer et al., 2005).

\subsubsection{Calculating temperature ramps}

To design temperature protocols for the demixing of binary liquids with a controlled time dependent driving $\xi$, eq. (2.8) is rearranged to give an equation for the slope of the temperature ramp

$$
\frac{\mathrm{d} T}{\mathrm{~d} t}=2 \xi(t) \Phi_{0}(T)\left(\frac{\mathrm{d} \Phi_{b}}{\mathrm{~d} T}\right)^{-1}
$$

For a constant driving $\xi$ one can take advantage of dimensionless units, i.e. to rescale the time with the time of one measurement $t_{\text {end }}$, so that the integration has to be done only once. Appropriate rescaling ${ }^{3}$ of time $\tau=t / t_{\text {end }}$ and ramp rates $\widetilde{\xi}=\xi t_{\text {end }}$ reveals that the integration has to be done only once for all $\xi$ values:

$$
\frac{\mathrm{d} T}{\mathrm{~d} \tau}=2 \widetilde{\xi} \Phi_{0}(T)\left(\frac{\mathrm{d} \Phi_{b}}{\mathrm{~d} T}\right)^{-1}
$$

In table 2.3 the $\widetilde{\xi}$ values ${ }^{4}$ are shown which match the boundary condition of $T_{\text {start }}=$ $25.80^{\circ} \mathrm{C}$ and $T_{\text {end }}=50.00^{\circ} \mathrm{C}$ for IBE $+\mathrm{W}$ and $T_{\text {start }}=33.50^{\circ} \mathrm{C}$ and $T_{\text {end }}=10.00^{\circ} \mathrm{C}$ for $\mathrm{M}+\mathrm{H}$.

\subsubsection{Influence of phase-diagram representation on driving $\xi$}

As described in section 2.2 there are two representations of the phase diagram, the first one uses a polynomial fit of the phase coexistence curve and was used for the

\footnotetext{
${ }^{2}$ Note that the signs in this formula have also been adjusted. Equation (2.8) presupposes that the composition is measured with respect to the majority phase of the droplets; otherwise a minus sign is needed. In other words, $\xi$ is positive if the temperature change drives the mixture deeper into the miscibility gap.

${ }^{3}$ Another choice of the dimensionless time is $\tau=\xi t$. Both choices differ by the factor of $\widetilde{\xi}$. Here I continue with the description how I actually implemented the temperature ramps although in retrospective view the next time I would take the other choice.

${ }^{4}$ The values of $\widetilde{\xi}$ are found by trial and error when integrating eq. (2.10).
} 


\begin{tabular}{lcc}
\hline IBE $+\mathrm{W}$ & poly & scaling \\
$\widetilde{\xi}_{r}$ & 1.03000 & 0.39205 \\
$\widetilde{\xi}_{l}$ & 0.60238 & 0.21347 \\
$\widetilde{\xi}_{0}$ & 1.63240 & 0.60552 \\
\hline $\mathrm{M}+\mathrm{H}$ & & \\
$\widetilde{\xi}_{r}$ & 0.71520 & 0.3282 \\
$\widetilde{\xi}_{l}$ & 0.42495 & 0.19904 \\
$\widetilde{\xi}_{0}$ & 1.14015 & 0.52724 \\
\hline
\end{tabular}

Table 2.3: $\widetilde{\xi}$ values for the calculation of the temperature ramps for the mixtures of IBE $+\mathrm{W}$ and $\mathrm{M}+\mathrm{H}$ and the polynomial and scaling representations of the phase diagrams.

calculation of temperature ramps, which were used for most of the experimental runs reported in this thesis. The second one uses a scaling fit, and would be the choice for a description from the theory side. By inspection of the phase diagrams given in fig. 2.2 there are only small differences in the compositions or temperatures. How would these differences translate in deviations in the driving?

The ratio of both drivings $\xi_{\text {scaling }}$ and $\xi_{\text {poly }}$ are independent of the driving strength:

$$
\frac{\xi_{\text {scaling }}}{\xi_{\text {poly }}}=\left(\frac{1}{2 \Phi_{0}^{s}} \frac{\mathrm{d} \Phi_{b}^{s}}{\mathrm{~d} T} \frac{\mathrm{d} T}{\mathrm{~d} t}\right)\left(\frac{1}{2 \Phi_{0}^{p}} \frac{\mathrm{d} \Phi_{b}^{p}}{\mathrm{~d} T} \frac{\mathrm{d} T}{\mathrm{~d} t}\right)^{-1}=\frac{\Phi_{0}^{p}}{\Phi_{0}^{s}} \frac{\mathrm{d} \Phi_{b}^{s}}{\mathrm{~d} T}\left(\frac{\mathrm{d} \Phi_{b}^{p}}{\mathrm{~d} T}\right)^{-1} .
$$

Here $\Phi^{s}$ and $\Phi^{p}$ denote the compositions in the scaling and poynomial representation of the phase diagram, respectively. This $\xi$-conversion factor depends only on temperature and is shown in fig. 2.4 as a function of the reduced temperature. Surprisingly, the $\xi$-conversion factor shows deviations up to a factor of two, although the scaling and polynomial representations in 2.2 differ not much.

To have consistent presentation of the data in this thesis, the values of $\xi$ for measurements which used temperature ramps based on the polynomial representation of the phase diagram are multiplied with the $\xi$-conversion factor $\xi_{\text {scaling }} / \xi_{\text {poly }}$.

\subsubsection{Calculating $\xi$ from measured temperature ramps}

The need for calculating the driving $\xi$ from the temperature log files of the experimental runs, came up when I checked the quality of time dependent driving, especially for periodic driving with a square wave.

An easy way is to just compare the temperatures. But for a real comparison, it is necessary to calculate the driving, $\xi$, from the measured temperatures in the experimental runs. This is done by evaluating eq. (2.8), which is not so straight forward because the derivative of noisy data (measured temperature ramp) is needed. 

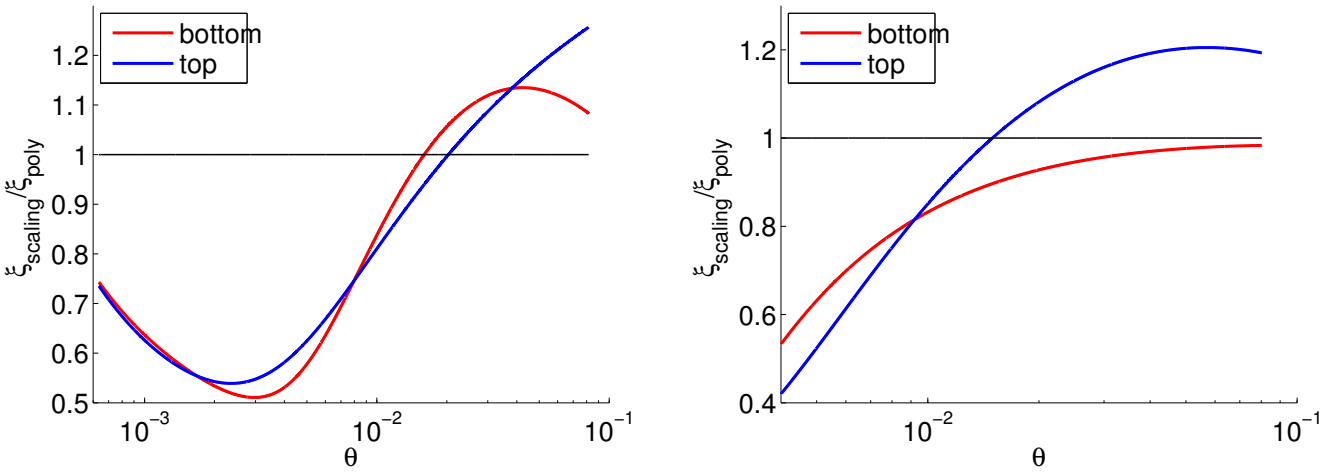

Figure 2.4: Conversion factor $\xi_{\text {scaling }} / \xi_{\text {poly }}$ for $\mathrm{IBE}+\mathrm{W}$ (left) and $\mathrm{M}+\mathrm{H}$ (right) in the lower (red) and upper layer (blue) of the sample.

There are some approaches to deal with the derivative of noisy data (Chartrand, 2011; Rudin et al., 1992; Stickel, 2010; Ahnert and Abel, 2007). For my purpose to estimate the derivative of the temperature ramp the approach of Chartrand (2011) worked best. Here the derivative $f^{\prime}=u$ is calculated by minimizing the following functional:

$$
F(u)=\alpha \int_{0}^{L}\left|u^{\prime}\right|+\frac{1}{2} \int_{0}^{L}|A u-f|^{2}
$$

$A$ is an integration operator. The first term is the regularisation term. It makes the derivative smooth. The second term penalizes discrepancy between the data $f$ and the reconstructed data $(A u)$ from its derivative $u$. $\alpha$ is a weighing factor: for high values the derivative is smooth and for small values the noisy derivative from finite-difference calculations is obtained. The minimization is done by evolving the corresponding Euler-Lagrange equation to stationarity ${ }^{5}$

$$
\frac{\mathrm{d} u}{\mathrm{~d} t}=\alpha \frac{\mathrm{d}}{\mathrm{d} x} \frac{u^{\prime}}{\left|u^{\prime}\right|}-A^{T}(A u-f)
$$

It is very useful to have the weighing factor $\alpha$, because in the beginning of the temperature ramps the noise is higher as compared to the end.

To deal with the decreasing level of noise the temperature ramp is cut into 10 pieces, for which the derivative is calculated with a value of $\alpha$, that decreases linearly between 100 and 1 . These parameters work well for ramp rates of $\xi=2 \times 10^{-5} \mathrm{~s}^{-1}$. They have been determined by eye.

\footnotetext{
${ }^{5}$ The implementation in Matlab is based on: http://octave.1599824.n4.nabble.com/Solving-largematrix-equations-td1635345.html
} 


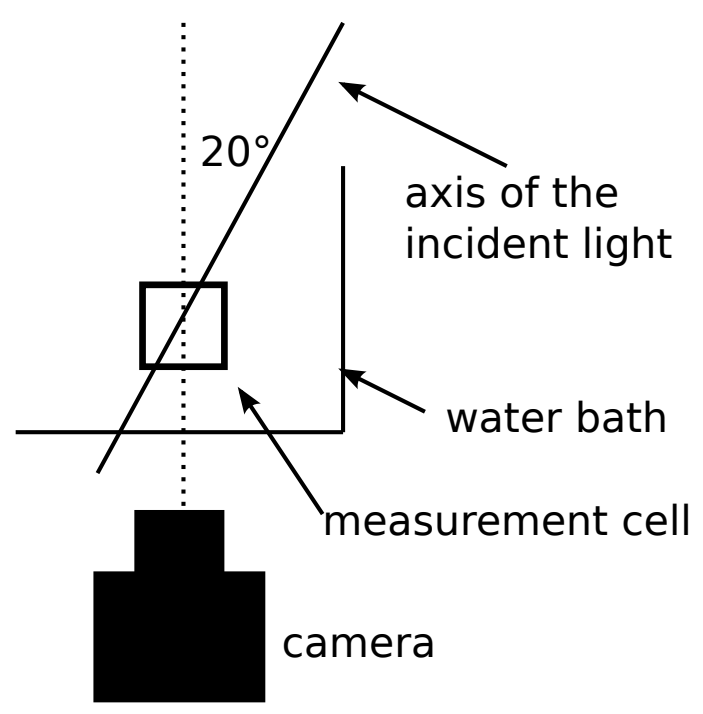

Figure 2.5: Setup for turbidity measurements. The measurement cell is immersed in the water bath to control the temperature. The light of a cold light source forms an angle of $20^{\circ}$ with the axis of the camera. If the mixture in the measurement cell is turbid light is scattered into the camera, providing bright pictures, whereas the images are dark when the mixture is clear.

\subsection{Turbidity measurements}

In this part I will describe the optical setup for the measurement of the turbidity and the analysis of the images leading to a space-time representation and the extraction of the oscillation periods. The idea and procedure was first described by Auernhammer et al. (2005). Starting point was the the setup for determining the transition temperature of binary mixtures (see section 2.2). It is used to extract the oscillation periods for the mixtures of $\mathrm{IBE}+\mathrm{W}$ and $\mathrm{M}+\mathrm{H}$ in various settings.

\subsubsection{Setup for turbidity measurements}

A sketch of the optical setup for the measurement is shown in fig. 2.5. The sample is illuminated by a KL 2500 LCD Schott cold light source in a dark field illumination. It turned out that an angle of about $20^{\circ}$ between the axis of illumination and camera provides optimal results. The camera is operated for this case with a commercial objective. 


\subsubsection{From turbidity to oscillation periods}

Here I describe the data processing from individual snapshots taken by the camera to the extraction of the oscillation period for the example of IBE+W subjected to a ramp rate $\xi=2.5 \times 10^{-5} \mathrm{~s}^{-1}$. In fig. 2.6(a)-(f) individual snapshots are shown. They clearly show an oscillating turbidity: some images are almost complete dark, while others show bright parts coming from the scattered light. The fact that there is not so much variation of the turbidity in horizontal direction, makes it possible to condense the time evolution of the turbidity in a space-time plot fig. 2.6(g). This is done by averaging the images in horizontal direction, leaving a line of the turbidity in vertical direction, which are put next to each other. Making a cut through the space-time plot gives an oscillating signal of the turbidity, fig. 2.6(h). The oscillation periods are extracted by detecting the maxima of the oscillating turbidity ${ }^{6}$. They are then conveniently presented in a form like in fig. 2.6(i).

\subsection{Measuring size distributions}

The experimental tools and subsequent image processing for determining the size distribution of droplets in binary phase separation were developed before. So I give only a very brief overview, a detailed description can be found in Rohloff (2011); Lapp (2011); Lapp et al. (2012).

In fig. 2.7 a sketch of the experimental setup is shown. The sample is illuminated by a mercury short arc lamp (LOT-Oriel 100W). A green filter (GF) selects the two green lines from the emission spectrum and lenses L1 and L2 form a light sheet. The camera detects the red filtered (RF) red fluorescence light coming from the emission of the droplets, labelled with the fluorescent dye Nile Red. With this technique the droplets will appear as bright disks on a dark background as shown in fig. 2.8(left). ${ }^{7}$ The radius and the position of the bright disks in the individual images are detected using the image processing toolbox of Matlab. If the time interval between the images is sufficiently small the droplets can be tracked through the image series. Since the droplets react to gravity by sedimentation according to the Stokes velocity, they are used as tracer particles to determine the flow field of the bulk fluid as reported in Lapp et al. (2012) (see fig. 2.8(left)). The information of position and radius of the droplets in each image makes it possible to display the time evolution of the size distribution as in fig. 2.8 (right).

\footnotetext{
${ }^{6}$ This procedure was used to detect the oscillation period for the experiments in chapter 6 while in chapter 5 the distance of two minima in the turbidity near the meniscus was used as the oscillation period.

${ }^{7}$ This is the case for the lower layer of IBE + W. In the upper layer Nile Red will dissolve preferentially in the bulk fluid, giving rise to dark disks on a bright background in the images.
} 

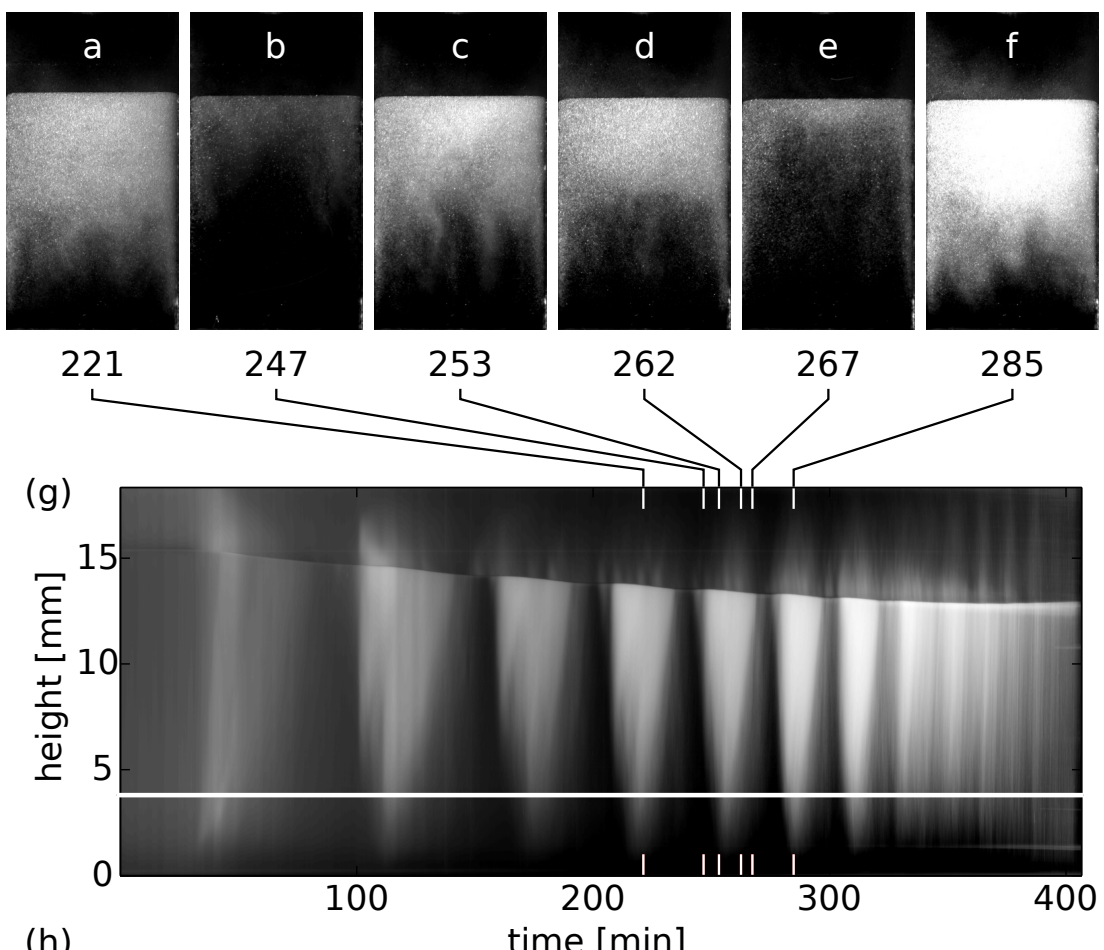

(h)

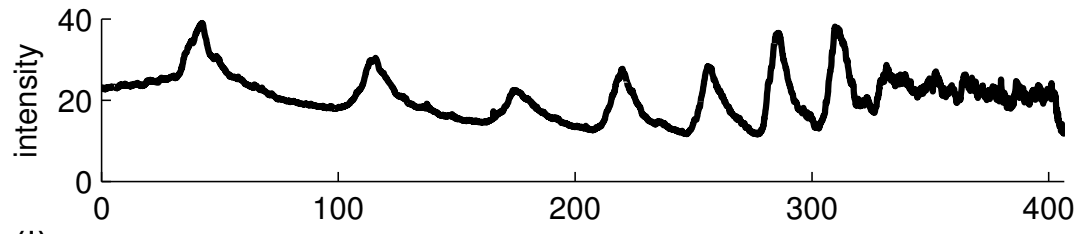

(i)

time [min]

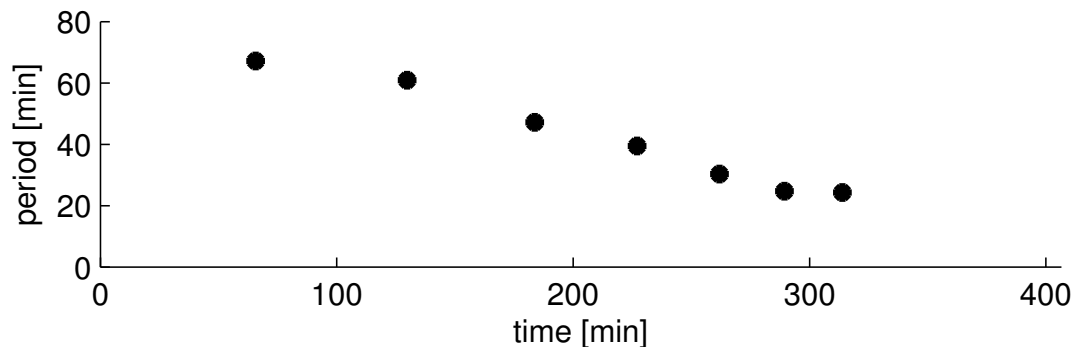

Figure 2.6: Snapshots and space-time plot showing the time evolution of the turbidity in a sample of IBE $+\mathrm{W}$ for a ramp rate $\xi_{l}=2.5 \times 10^{-5} \mathrm{~s}^{-1}$. Panels (a)-(f) show snapshots of the sample. The cell has a cross section of $10 \times 10 \mathrm{~mm}^{2}$ and snapshots are provided in the true aspect ratio. The turbidity evolves mainly in the lower layer. Above the upper layer, there is air, which is not shown. In panel $(\mathrm{g})$ the turbidity information of the snapshots is condensed in a space-time plot, where the height corresponds to the height of the snapshots shown in panels (a)-(f). (h) Section through the space-time plot at a height of $4 \mathrm{~mm}$, i.e. along the white line indicated in panel (g). (i) Oscillation period $\Delta t_{i}$ extracted from the oscillating turbidity as the time between between the $i^{\text {th }}$ and the $(i+1)^{\text {st }}$ maximum. 


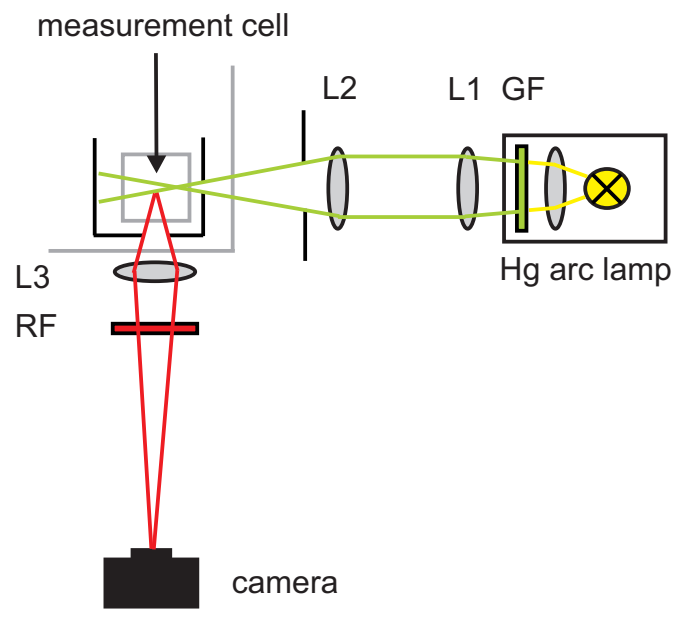

Figure 2.7: Experimental setup showing the optics for the determination of droplet size distributions. Reproduced from Rohloff (2011).
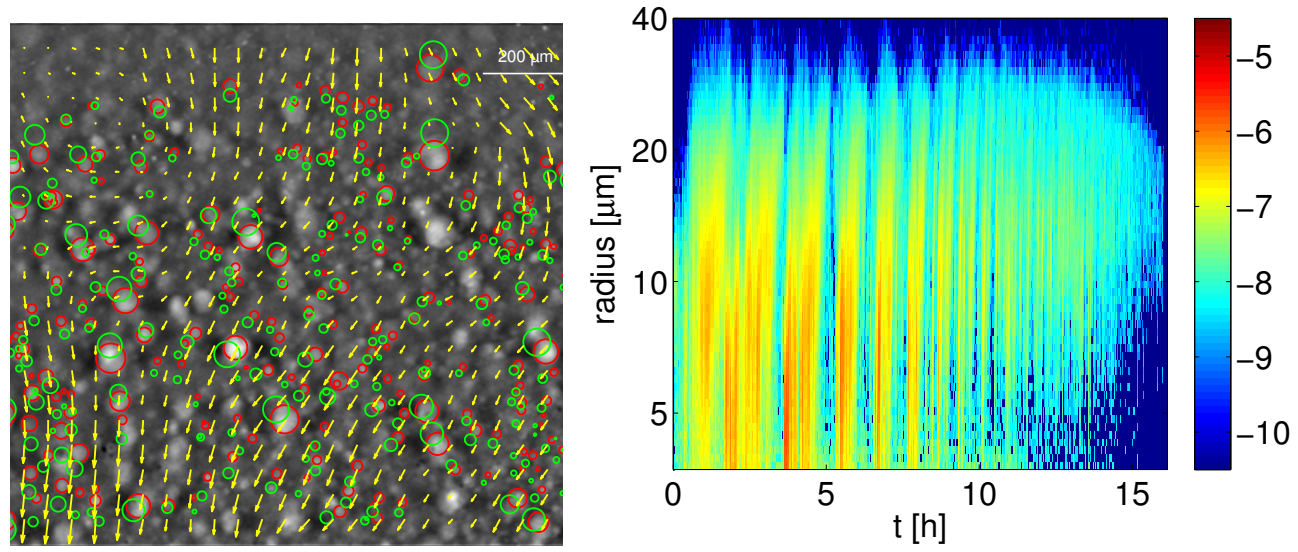

Figure 2.8: Detected droplets and the velocity field of the background flow and the evolution of the size distribution for the lower layer of IBE $+\mathrm{W}$ and a ramp rate of $\xi_{l}=1.05 \times 10^{-5} \mathrm{~s}^{-1}$. Left: Snapshot at $t=2.76 \mathrm{~h}$ with the detected droplets (red) and positions in the subsequent image (green). The displacement field of the bulk flow is shown by yellow arrows. Right: The number density of droplets per radius is given in $\mu \mathrm{m}^{-4}$ and color coded on a logarithmic scale. Images are reproduced from Rohloff (2011). 


\section{Numerical methods}

In this chapter I describe numerical methods to investigate competitive growth in an assembly of aggregates. In the literature there are at least two approaches to model the evolution of the aggregate sizes. One is the formulation of kinetic equations as described for example in Slezov and Schmelzer (1994). The other approach is to develop and integrate mean-field models. This latter approach was adopted in Yao et al. (1993) to investigate Ostwald ripening. In the present work I also follow this latter approach: I modify the mean-field description of Ostwald ripening to incorporate the effects of external material flux.

I consider an assembly of aggregates undergoing competitive growth with a sustained overall volume growth, due to a material flux into the system. The discussion is based on our paper Vollmer et al. (2014). Each member of the assembly is described by its radius $R_{i}, i=1 \cdots N$, which obeys (see section 1.4 )

$$
\dot{R}_{i}=\frac{D \sigma}{R_{i}^{2}}\left(k \frac{R_{i}}{\langle R\rangle}-1\right), \quad k=1+\frac{\xi \mathcal{V}}{4 \pi D \sigma N} .
$$

Here the diffusive accretion of material of the $N$ aggregates is described by the term $D \sigma$ and $k$ characterises the strength of growth coming from the material flux, $\xi$, into the assembly compared to a pure ripening scenario, where the total volume is conserved. $\mathcal{V}$ is the sample volume and $\langle R\rangle=\frac{1}{N} \sum_{i} R_{i}$ the average radius of the aggregate assembly.

The growth of aggregates is coupled by the occurrence of the zeroth and first moment of their size distribution in the equation of motion eq. (3.1), i.e., by the number of aggregates $N$ and the average radius $\langle R\rangle$. Since aggregates can evaporate/dissolve the number of aggregates is not constant in general. There are some limits where an analytic treatment is possible (see section 4.2 for the large $k$ limit and section 1.3 for $k=1$ ). For all other cases one has to rely on the numerical integration of the eq. (3.1).

The first part of this chapter is concerned with the nondimensionalisation of eq. (3.1), and the second part with the initial distributions, whose evolution I have investigated. In the final part I describe the integration scheme adopted to follow the aggregate size distribution of such an assembly in time. 


\subsection{Dimensionless units}

\subsubsection{Time and length scales}

In the following I concentrate on the case of a non-vanishing and constant driving $\xi$. The case of Ostwald ripening, where $\xi=0$, will emerge as a limiting case to be discussed later. I consider a setting, where the total volume $V$ of the aggregates changes linearly in time. So it is straight forward to measure the time in terms of the total volume of the aggregate assembly. The volume change is characterised by the change in aggregate volume fraction $\xi$ so that

$$
\frac{\mathrm{d} V}{\mathrm{~d} t}=\mathcal{V} \xi
$$

Let $V(t=0)=V_{0}$, then the integration of equation 3.2 leads to

$$
V(t)=V_{0}+\mathcal{V} \xi t
$$

The total volume will be measured with respect to the initial volume $V_{0}$. A time scale $t_{0}$ for measuring time is defined as the time until the initial volume has doubled (see fig. 3.1):

$$
2 V_{0}=V\left(t_{0}\right)=V_{0}+\mathcal{V} \xi t_{0} \quad \Rightarrow \quad V_{0}=\mathcal{V} \xi t_{0}
$$

With the definition of $k=1+\frac{\xi \mathcal{V}}{4 \pi D \sigma N}$ the time scale $t_{0}$ is expressed in terms of $k_{0}$ and $N_{0}$ :

$$
t_{0}=\frac{V_{0}}{\mathcal{V} \xi}=\frac{V_{0}}{4 \pi D \sigma N_{0}\left(k_{0}-1\right)}
$$

Note that $t_{0}$ is negative for a decreasing overall volume $(\xi<0)$. So volume and time scales are related like:

$$
\frac{V(t)}{V_{0}}=\frac{V_{0}+\mathcal{V} \xi t}{V_{0}}=1+\frac{t}{t_{0}} \quad \Rightarrow \quad \frac{t}{t_{0}}=\frac{V(t)}{V_{0}}-1 .
$$

Since time is measured in terms of the overall volume, this also translates a time derivative into a volume derivative. The time derivative of a quantity $X$ with respect to the rescaled time $t / t_{0}$ is:

$$
\frac{\mathrm{d} X}{\mathrm{~d} t} t_{0}=\frac{\mathrm{d} X}{\mathrm{~d} V} \frac{\mathrm{d} V}{\mathrm{~d} t} t_{0}=\frac{\mathrm{d} X}{\mathrm{~d} V} V_{0} \quad \text { or } \quad \frac{\mathrm{d} X}{\mathrm{~d} t}=\frac{\mathrm{d} X}{\mathrm{~d} V} \cdot \frac{V_{0}}{t_{0}} \stackrel{3.5}{=} \frac{\mathrm{d} X}{\mathrm{~d} V} 4 \pi D \sigma N_{0}\left(k_{0}-1\right)
$$

where the derivative of eq. (3.6) $\left(\frac{\mathrm{d} V}{\mathrm{~d} t}=\frac{V_{0}}{t_{0}}\right)$ is used. When time is measured in terms of the overall volume, eq. (3.6), the time scale $t_{0}$ depends on the initial value of $k$. For comparing the evolution of aggregate assemblies with different $k_{0}$, it is useful to 


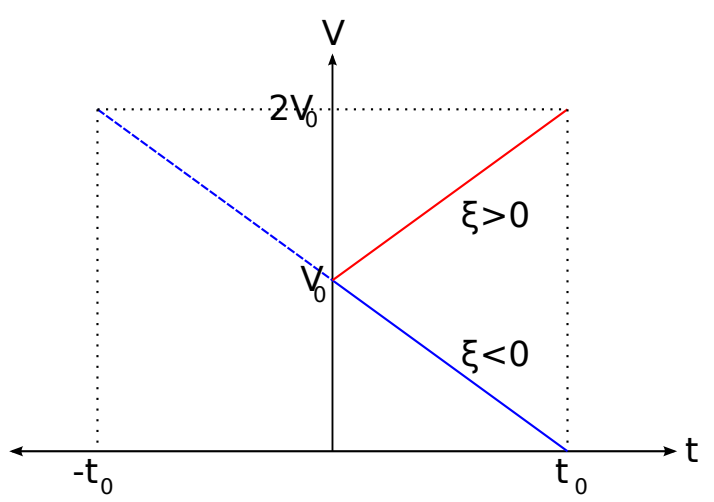

Figure 3.1: Sketch for the definition of the time scale $t_{0}$ in the cases of $\xi>0$ in red and $\xi<0$ in blue. The dashed line describes an extrapolation of the volume of the aggregate assembly into the past to a total volume of $2 V_{0}$.

define a dimensionless time $\tau$ which does not depend on $k_{0}$. With eq. (3.5) I get

$$
\tau=\frac{t}{\left(k_{0}-1\right) t_{0}}=t \cdot \frac{4 \pi D \sigma N_{0}}{V_{0}}
$$

The length scale $L$ is derived from the initial volume $V_{0}$ as the volume averaged initial radii of the aggregates:

$$
V_{0}=\frac{4 \pi}{3} N_{0}\left\langle R(t=0)^{3}\right\rangle \quad \Rightarrow \quad L:=\left\langle R(t=0)^{3}\right\rangle^{1 / 3}=\left(\frac{3}{4 \pi} \frac{V_{0}}{N_{0}}\right)^{1 / 3}
$$

Moreover, the dimensionless ramp rate $\xi$ is

$$
\xi=\frac{\xi \mathcal{V}}{4 \pi D \sigma} \quad \text { such that } \quad k=1+\frac{\xi}{N} .
$$

\subsubsection{Dimensionless equation}

To write eq. (3.1) in dimensionless units

$$
x_{i}=\frac{R_{i}}{L} \quad \text { and } \quad \tau=\frac{t}{\left(k_{0}-1\right) t_{0}}
$$


are chosen as the dimensionless radius $x_{i}$ of aggregate $i$ and time $\tau$. Inserting this into eq. (3.1) and using the definition of $t_{0}$ in eq. (3.5), leads to

$$
\begin{aligned}
\frac{\mathrm{d} x_{i}}{\mathrm{~d} \tau} & =\frac{\mathrm{d} R_{i}}{\mathrm{~d} t} \frac{\left(k_{0}-1\right) t_{0}}{L}=\frac{D \sigma}{x_{i}^{2} L^{2}} \frac{\left(k_{0}-1\right) t_{0}}{L}\left(k \frac{x_{i}}{\langle x\rangle}-1\right) \\
& =\frac{1}{3 x_{i}^{2}}\left(k \frac{x_{i}}{\langle x\rangle}-1\right) \frac{1}{L^{3}} \underbrace{\frac{3 V_{0}}{4 \pi N_{0}}}_{L^{3}} .
\end{aligned}
$$

For the cube of the aggregate radius $Q_{i}=x_{i}^{3}$ we thus find the evolution equation

$$
\frac{\mathrm{d} Q_{i}}{\mathrm{~d} \tau}:=\frac{\mathrm{d} x_{i}^{3}}{\mathrm{~d} \tau}=k \frac{x_{i}}{\langle x\rangle}-1
$$

\subsection{Initial distributions}

In this part I describe the initial distributions used for the integration and show how to compute them. The initial distributions can be calculated analytically if the inverse of the cumulative distribution function (CDF) of the desired probability density function (PDF) is known. For other distributions the inverse has to be calculated numerically or by interpolating a lockup table of the function. The CDF $\mathcal{C}(R)$ of the corresponding PDF $P(R)$ is obtained by integration

$$
\mathcal{C}(R)=\int_{R_{\text {min }}}^{R} P(R) \mathrm{d} R
$$

This is a monotonic function rising from $\mathcal{C}(R)=0$ for $R \leq R_{\min }$ to $\mathcal{C}(R)=1$ for $R \geq R_{\max }$. To get a list of $N_{0}$ aggregate radii the CDF has to be discretised. This is done by identifying $\mathcal{C}=(i-1) /\left(N_{0}-1\right)$ with $i=1 \cdots N_{0}$. Solving for $R$ then leads to the desired list of aggregate sizes which can be used as the initial distribution. For the following distributions the equations for the calculation of the initial distribution are given explicitly, because they are used throughout the thesis.

\subsubsection{Uniform distribution}

The uniform distribution of $R \in\left[R_{\min }, R_{\max }\right]$ has the following PDF and CDF

$$
P(R)=\frac{1}{R_{\max }-R_{\min }}, \quad \mathcal{C}(R)=\frac{R-R_{\min }}{R_{\max }-R_{\min }} .
$$


The corresponding initial condition, obtained by inverting the descretised CDF, is

$$
R_{i}=R_{\min }+\left(R_{\max }-R_{\min }\right) \frac{i-1}{N_{0}-1}
$$

The resulting distributions are shown in fig. 3.2 for $R_{\min }=0.05$ and $R_{\max }=7.92$.
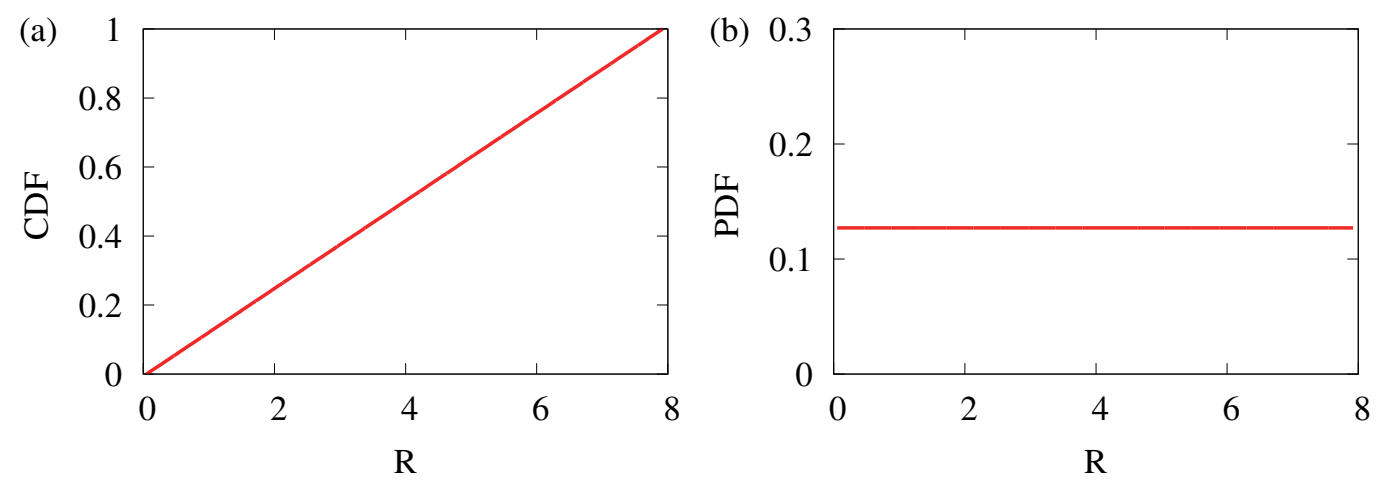

Figure 3.2: Uniform initial distribution for $R_{\min }=0.05$ and $R_{\max }=7.92$. (a) CDF and (b) PDF.

\subsubsection{Bell-shaped distribution}

A bell shaped distribution with $R \in[0,2 \bar{R}]$ is obtained from the hyperbolic cosine function

$$
\begin{aligned}
P(R) & =\frac{1}{2 \tanh (\bar{R})} \frac{1}{\cosh ^{2}(R-\bar{R})} \\
\mathcal{C}(R) & =\frac{1}{2}\left(\frac{\tanh (R-\bar{R})}{\tanh (\bar{R})}+1\right) .
\end{aligned}
$$

The corresponding initial distribution is then given by

$$
R_{i}=\bar{R}+\operatorname{artanh}\left(\tanh (\bar{R})\left(2 \frac{i-1}{N_{0}-1}-1\right)\right)
$$

In fig. 3.3 the resulting distributions are shown for $\bar{R}=4.84$.

\subsubsection{Bimodal distribution}

The bimodal distribution is built in a similar way as the bell-shaped distribution. Here I use the superposition of two inverse squared hyperbolic cosine functions for 

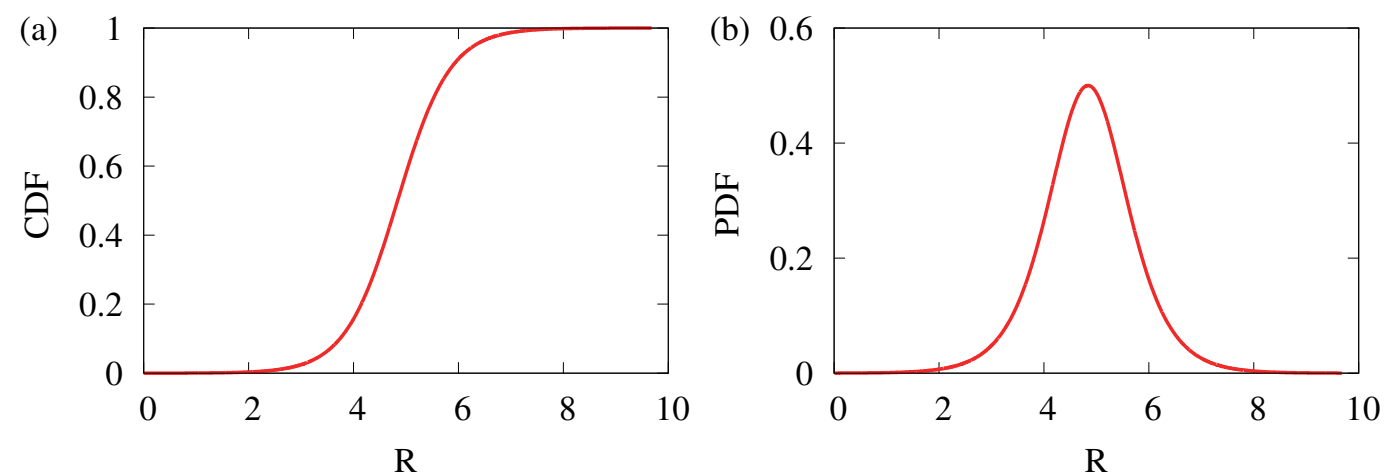

Figure 3.3: Bell-shaped initial distribution for $\bar{R}=4.84$. (a) CDF and (b) PDF.
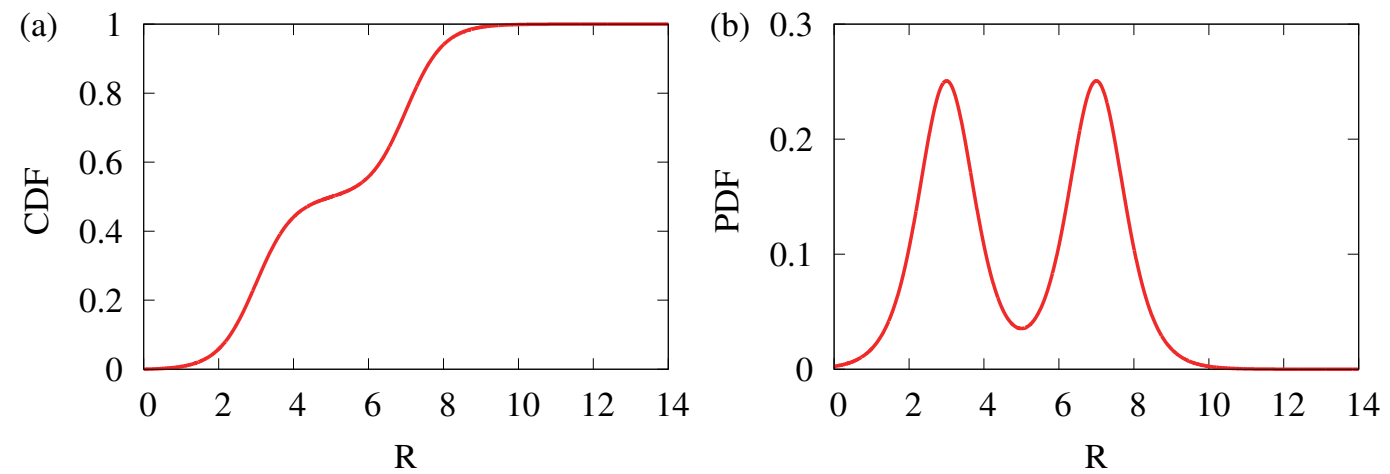

Figure 3.4: Bimodal initial distribution for $R_{1}=3$ and $R_{2}=7$. (a) $\mathrm{CDF}$ and (b) PDF

the PDF for $R \in\left[0,2 R_{2}\right]$

$$
\begin{aligned}
& P(R)=\frac{1}{\tanh \left(2 R_{2}-R_{1}\right)+2 \tanh \left(R_{2}\right)+\tanh \left(R_{1}\right)}\left(\frac{1}{\cosh ^{2}\left(R-R_{1}\right)}+\frac{1}{\cosh ^{2}\left(R-R_{2}\right)}\right) \\
& \mathcal{C}(R)=\frac{\tanh \left(R-R_{1}\right)+\tanh \left(R-R_{2}\right)+\tanh \left(R_{1}\right)+\tanh \left(R_{2}\right)}{\tanh \left(2 R_{2}-R_{1}\right)+2 \tanh \left(R_{2}\right)+\tanh \left(R_{1}\right)} .
\end{aligned}
$$

In fig. 3.4 the PDF and $\mathrm{CDF}$ are shown for $R_{1}=3$ and $R_{2}=7$. 


\subsection{Integration scheme}

The integration of the $N$ coupled ordinary differential equations (3.13) is done numerically by a self written routine in C. Integrating the cubes, $Q_{i}$, of the respective radii according to eq. (3.13) avoids numerical instabilities for very small radii, which would arise for the integration of eq. (3.12) due to a diverging $\mathrm{d} x_{i} / \mathrm{d} \tau$ for zero radius. The evaporation of aggregates is treated self-consistently, so that the overall volume grows linearly in time. This is achieved by updating the volumes $Q_{i}$ via a predictorcorrector scheme. The volume of the evaporated aggregates is recursively transferred to the surviving aggregates, thus ensuring that the overall volume will grow linearly in time. For the bookkeeping of the evaporating aggregates it is used that the order of aggregate sizes will not change in time, if they are ordered according to their sizes. This is an immediate consequence of $k>0$ and the structure of eq. (3.13): Consider two aggregates of different sizes with $Q_{i}>Q_{j}$ then

$$
\frac{\mathrm{d}}{\mathrm{d} \tau}\left(Q_{i}-Q_{j}\right)=\frac{k}{\langle x\rangle}\left(x_{i}-x_{j}\right)>0 .
$$

Hence, the difference in size will grow in time, which preserves the order among the aggregates.

The integration scheme is depicted in fig. 3.5. Starting point of the integration of an initial distribution (see section 3.2) is the total volume increment $\delta V$ which translates into a time increment $\delta \tau$ :

$$
\delta V=\sum_{i=1}^{N} \delta x_{i}^{3} \stackrel{3.13}{=} N(k-1) \delta \tau \stackrel{3.10}{=} \xi \delta \tau .
$$

Then $k$ and $\langle x\rangle$ are calculated from the zeroth and first moment of the aggregate size distribution and all aggregate volumes $Q_{i}$ are updated according to a discrete version of eq. (3.13)

$$
Q_{i}(\tau+\delta \tau)=Q_{i}(\tau)+\left(k \frac{x_{i}}{\langle x\rangle}-1\right) \delta \tau .
$$

Now every aggregate is checked if it has evaporated during the integration step, i.e. $Q_{i}(\tau+\delta \tau) \leq 0$. In this case these aggregates are taken out, the number of aggregates is updated and the initial volume of the evaporated aggregates is added to the initial volume increment $\delta V$. This volume will also be redistributed among the surviving aggregates. This loop is repeated until no aggregate evaporates in the proposed integration step. Aggregate radii and time are updated and the next integration step is done, until the final overall volume is reached.

To minimize inaccuracies coming from the treatment of the evaporation and the 


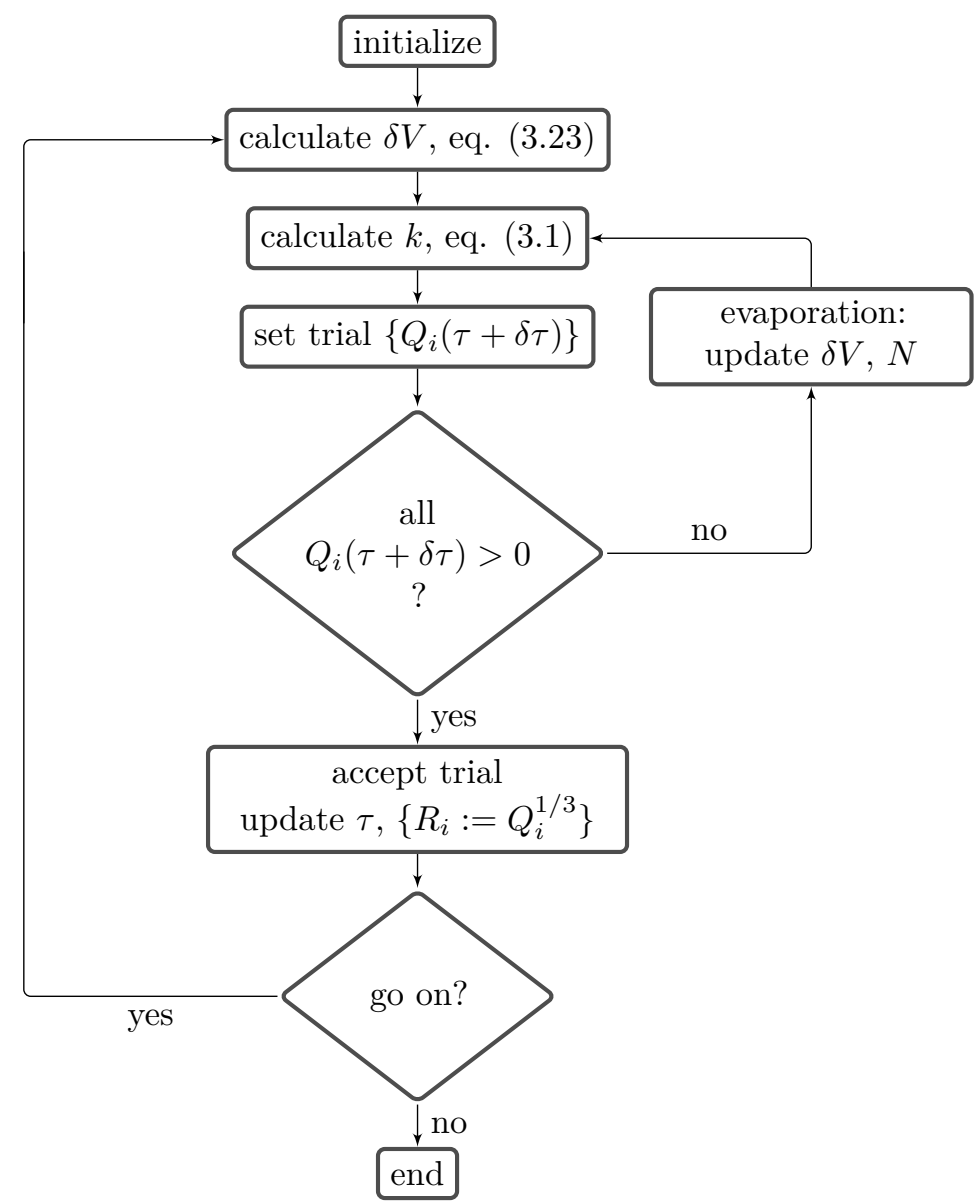

Figure 3.5: Integration scheme for the integration of an assembly of aggregates $\left\{R_{i}\right\}$. The aggregate number $N$, the volume increment $\delta V$ and $k$ are adjusted self-consistently when aggregates evaporate. 
redistribution of the volume, the integration step has to be small enough, that not more than one aggregate will evaporate in each integration step. To guarantee that and allowing the algorithm to perform at a sufficient speed, an adaptive time stepping is implemented. To avoid the overhead of adaptive step size control, the time steps are chosen equidistant on a logarithmic scale for an assembly with a growing overall volume $(k>1)$. Thus a certain number of integration steps is needed to increase the volume by one order of magnitude and allows the integration to run long enough to study asymptotic behaviour. For a decreasing overall volume $(k<1)$ the time steps are chosen to be constant, since the number of steps is limited anyway by $V_{\text {end }}=0$. The self-consistent treatment of the evaporation introduces a small error, because the smallest aggregate is not followed exactly to zero size. Rather the remaining volume in that integration step is redistributed among all other aggregates. In this sense the integration does not follow exactly eq. (3.13). This error is small for a large number of aggregates and a large $\mathrm{k}$ value. To be exact here, the smallest aggregate has to be followed by adaptive integration steps to zero size. Then the number of aggregates has to be decreased by one and the integration can be continued in the same way until the next aggregate evaporates.

This would also be the way to treat the Ostwald ripening case, where $V=$ const and therefore $\xi=0$. Here the integration scheme depicted in fig. 3.5 cannot be followed because $\delta V=0$. So the integration of the assembly has to be done from one evaporating aggregate to another one and decreasing the number in between. Choosing a fixed number of integration steps between to evaporation events will then lead to a very similar efficient integration of the evolution of the assembly. 



\section{Size Distributions}

In the present chapter I investigate how the size distribution of an assembly evolves when its overall volume grows in time. In chapter section 4.1 I present numerical data for three different initial distributions. This discussion is based on the numerical methods developed in chapter 3. In section 4.2 a theory is developed for the evolution of the size distribution in the limit of large values of $k$. The theory is compared to the evolution of the uniform initial size distribution. This part is based on our paper Vollmer et al. (2014). So far the discussion focussed on the theoretical description of the evolution of aggregate size distributions in the absence of gravity. In section $4.3 \mathrm{I}$ discuss the evolution of size distributions measured during continuously driven phase separation in binary mixtures. In the experiments gravitational settling dominates the evolution of the size distribution for droplets larger than Brownian particles. Hence, a growth law for large droplets is established based on the collection of smaller droplets by large droplets during gravitational settling. The resulting size distribution matches with the distributions measured experimentally.

\subsection{Ripening with overall volume growth: numerical results}

In fig. 4.1 the evolution of the size distribution is shown for four different values of $\xi$, which correspond to the initial values of $k=5,10,50$ and 100. To explore the impact of the shape of the initial condition, I show the evolution for three different initial conditions: in fig. 4.1(a) the evolution for a bell-shaped initial distribution according to eq. (3.18), in fig. 4.1(b) a bimodal distribution (eq. (3.21)) and in fig. 4.1(c) a uniform initial distribution (eq. (3.15)). For all distributions the probability density function $(\mathrm{PDF})$ is shown as a function of the radius of the aggregates, normalized by the mean value of the respective initial distribution. In each panel of fig. 4.1 there are four quadruples of functions, that show the time evolution for the four different initial $k$ values. The initial PDF is shown on the very left by a black line. The three subsequent quadruples correspond to later times, when the overall volume of the aggregate assembly has increased by a factor of 10 , respectively so that the right quadruples show the assemblies for an overall volume, that amounts to 1000 times the initial volume. Depending on the shape of the distribution the initial volumes of the corresponding assemblies are different: the initial volume for the bell-shaped distribution is 1.103, for the bimodal distribution 1.586 and for the uniform distribution 1.976, respectively. Since the volume of the distribution in 


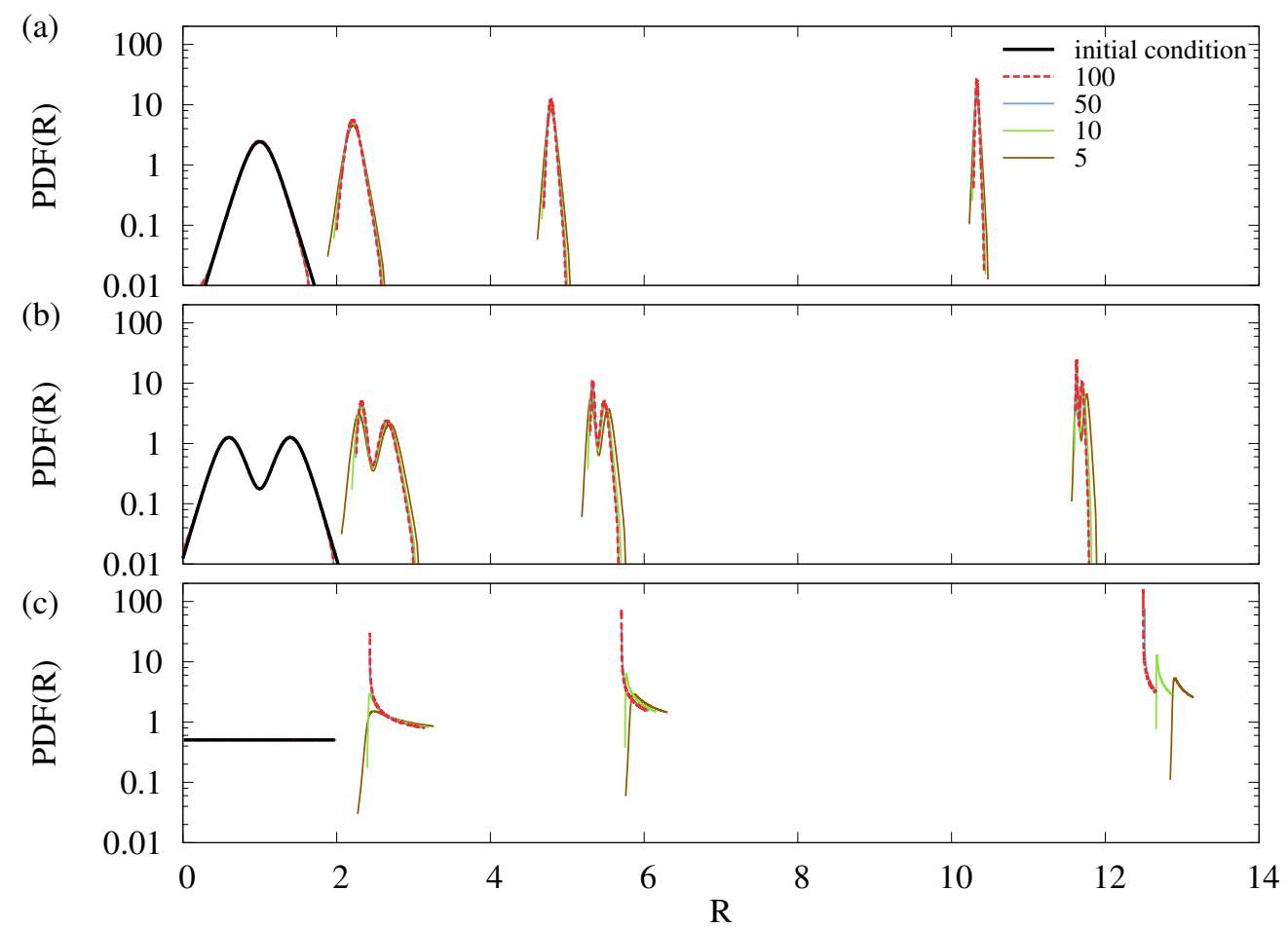

Figure 4.1: Evolution of the PDF for a bell-shaped (a), a bimodal (b) and a uniform initial condition (c). For better comparison the initial conditions (black lines) are chosen such, that the mean value is one. The subsequent three quadruples of lines correspond to the PDF at times, where the overall volume has increased by a factor of 10 , respectively. The initial $k$ value is color coded as indicated by the legend.

fig. 4.1 is increased by a factor of 10 as time proceeds, the mean radius becomes larger for the larger initial volume, if one compares the position of the different distributions. All distributions in one quadruple are shown for the same overall volume of the aggregate assembly, i.e. for the same dimensionless time in the simulations. At this point we make five observations that will be further substantiated in the following section.

- The width of the distribution decreases as time evolves, i.e. the distribution become more and more monodisperse. This is best seen for the bell-shaped distribution (fig. 4.1(a)), but one easily verifies this behaviour also for the other distributions.

- At early time the distributions for $k=5$ and 10 in fig. 4.1(c) develop a tail toward the small aggregates, and they feature larger average aggregate sizes 
at late times. This is a hallmark of the evaporation of aggregates. The tail is due to aggregates that shrink and evaporate when their radius approaches zero. The larger average size is required to achieve the prescribed overall volume with a smaller number of aggregates.

- The PDF for $k=50$ and 100 look almost the same. Indeed this holds for the uniform initial distribution in fig. 4.1(c) for all $k \gtrsim 50$, where no aggregates evaporate. The shape for the different initial $k$ values for the bell-shaped and the bimodal distributions differ not so much, because here a much smaller fraction of aggregates evaporate as compared to fig. 4.1(c).

- From the inspection of the numerical data one verifies that for all $k>1$ the growth at late times proceeds at a fixed aggregate number. Subsequently, evolution proceeds at a fixed value of $k$, and the difference in shape with respect to the PDF for larger values of $k$ does not evolve any longer.

- Typical features of the distributions are preserved for all times, e.g. the two peaks of the bimodal distribution. We do not observe a relaxation to an universal distribution.

The evolution of the size of individual aggregates and their evaporation is discussed in section 4.2.2. In section 4.2.3 I address the time evolution of the size distributions. To gain further insight in the evolution of the size distributions it is instructive to show them as a function of the reduced radius $R /\langle R\rangle$, see fig. 4.2. Here the distributions are shown for three different times: the initial time (left), intermediate time, where the overall volume has increased by a factor of 10 (middle) and for late time, where the overall volume has increased by a factor of 100 (right). We clearly see from fig. 4.2, that the distributions become narrower as time goes on, as already observed in fig. 4.1. Note that the range of the radius axis has been rescaled for better visibility in fig. 4.2. In contrast the universal asymptotic distribution of the LSW theory which describes the evolution of an aggregate assembly for $k=1$ (constant overall volume) stays the same (Lifshitz and Slyozov, 1961):

$$
p\left(\frac{R}{\langle R\rangle}\right)=\frac{3^{4} 2^{-5 / 3} e\left(\frac{R}{\langle R\rangle}\right)^{2} \exp \left(-\frac{1.5}{1.5-R /\langle R\rangle}\right)}{\left(\frac{R}{\langle R\rangle}+3\right)^{7 / 3}\left(\frac{3}{2}-\frac{R}{\langle R\rangle}\right)^{11 / 3}} \quad \text { for } \quad 0 \leq \frac{R}{\langle R\rangle} \leq \frac{3}{2} .
$$

We already observed that the evolution of the distributions looks almost the same for initial values of $k \gtrsim 50$. For $k \gg 1$ the evolution equation, eq. (1.10), of the 

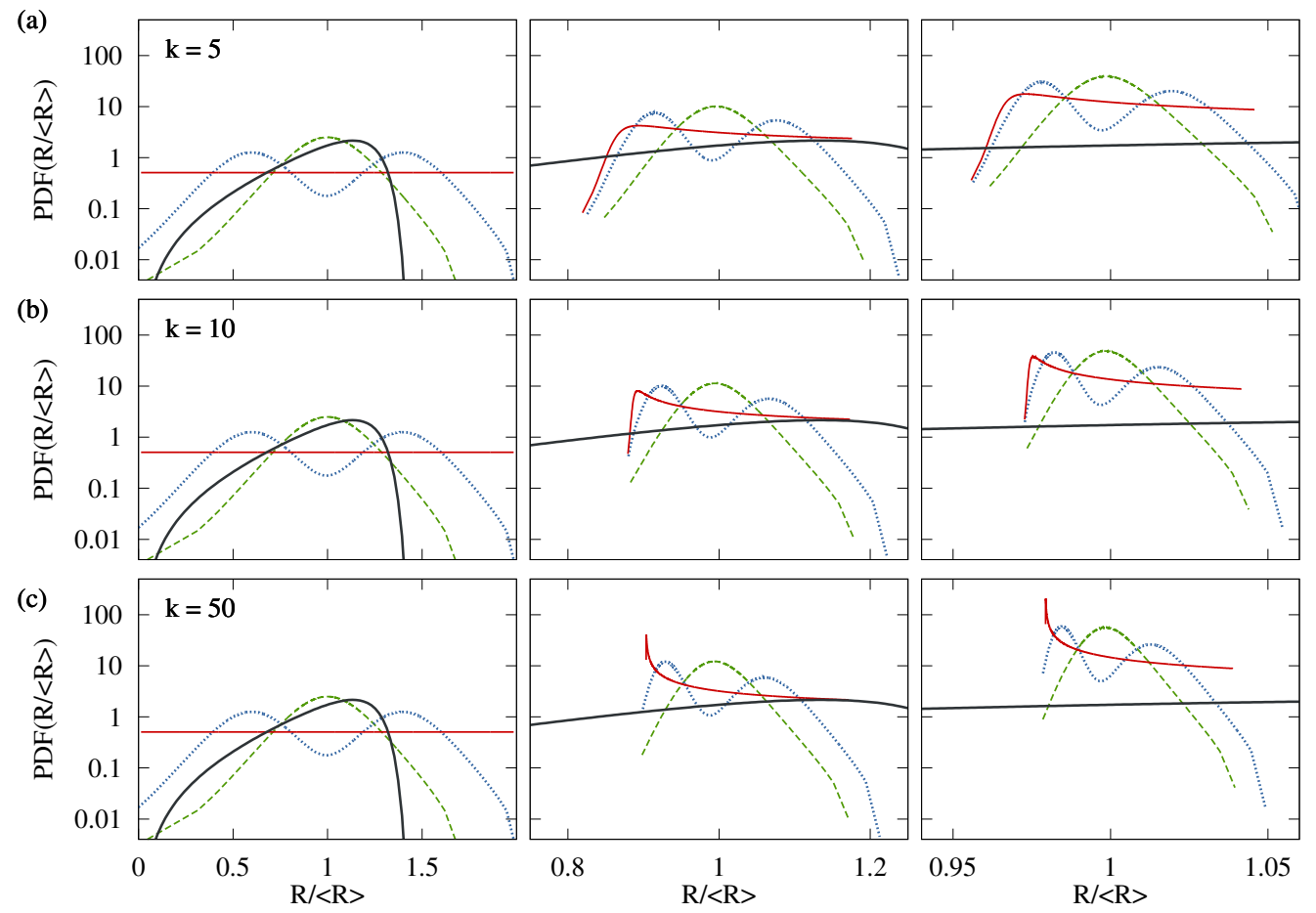

Figure 4.2: Evolution of the PDF for initial $k$ values of (a) $k=5$, (b) $k=10$ and (c) $k=50$, respectively. The different shapes of the distributions are color coded: bell-shaped (dashed green), bimodal (dotted blue) and uniform (solid red). The distributions are given as a function of the reduced radius $R /\langle R\rangle$. The left panels show the initial distribution. The middle and right panels show the distributions at times where the overall volume has increased by a factor of 10 and 100 respectively. In all panels the universal distribution of LSW theory eq. (4.1) is provided as a solid black line. Beware, the different ranges of the radius axis. The range is decreased by a factor of 4 between the respective plots. 
aggregate assembly is easily integrated:

$$
\begin{aligned}
\dot{R} & =\frac{D \sigma}{R^{2}}\left(k \frac{R}{\langle R\rangle}-1\right) \\
\dot{R} & \approx \frac{D \sigma}{R} \frac{k}{\langle R\rangle} \quad \text { for } \quad k \gg 1 \\
\frac{\mathrm{d}}{\mathrm{d} t} R^{2} & =\frac{2 D \sigma k}{\langle R\rangle}=f(t)
\end{aligned}
$$

The time derivative of $R^{2}$ in eq. (4.4) has to be only a function of time. If this time dependent function is chosen to be the time derivative of $\left\langle R^{2}\right\rangle$, then

$$
R^{2}-\left\langle R^{2}\right\rangle=\mathrm{const}
$$

is a constant of motion. This suggests to plot the size distributions as a function of $R^{2}-\left\langle R^{2}\right\rangle$. Indeed we see in fig. 4.3, that the size distributions for $k=50$ and 100 collapse for all times including the initial distribution when they are given as a function of $R^{2}-\left\langle R^{2}\right\rangle$. For $k=5$ and 10 there is a broadening of the distribution in this coordinates. However, this is expected since we derived the constant of motion in the limit of $k \gg 1$.

From eq. (4.3) it is easy to see why the distribution become narrower in the course of time. The growth rate $\dot{R}$ is a monotonically decreasing function of the radius, i.e the smaller aggregates grow faster than the larger ones. This exactly leads to the narrowing of the size distribution. For the synthesis of monodisperse particles this observation goes back to Reiss (1951). This mechanism is also discussed in the context of diffusively growing cloud droplets (Wallace and Hobbs, 2006) and in designing synthesis routs for monodisperse size distributions in material science (Yin and Alivisatos, 2005; Radmilovic et al., 2011; Hoyt, 2011).

As a summary, we gained a qualitative understanding of the impact of $k$, that describes the strength of an external material flux onto the aggregates of the assembly. We observed ceasing of evaporation, size focussing and no relaxation to a universal size distribution. In view of eq. (4.3) it is promising to gain insight of the dynamics from an analytic treatment in the limit of large $k$-values. On the other hand there is also need to understand the evolution of the size distribution for values of $k \approx 3 \cdots 5$, because this is a typical range of experimental conditions in the synthesis of monodisperse colloids (see fig. 1.5(c) and section 7.1).

\subsection{Theory for large $k$ values}

In this section a quantitative understanding of the evolution of the size distribution for large $k$ is developed based on an analytic treatment of the equation of motion of 

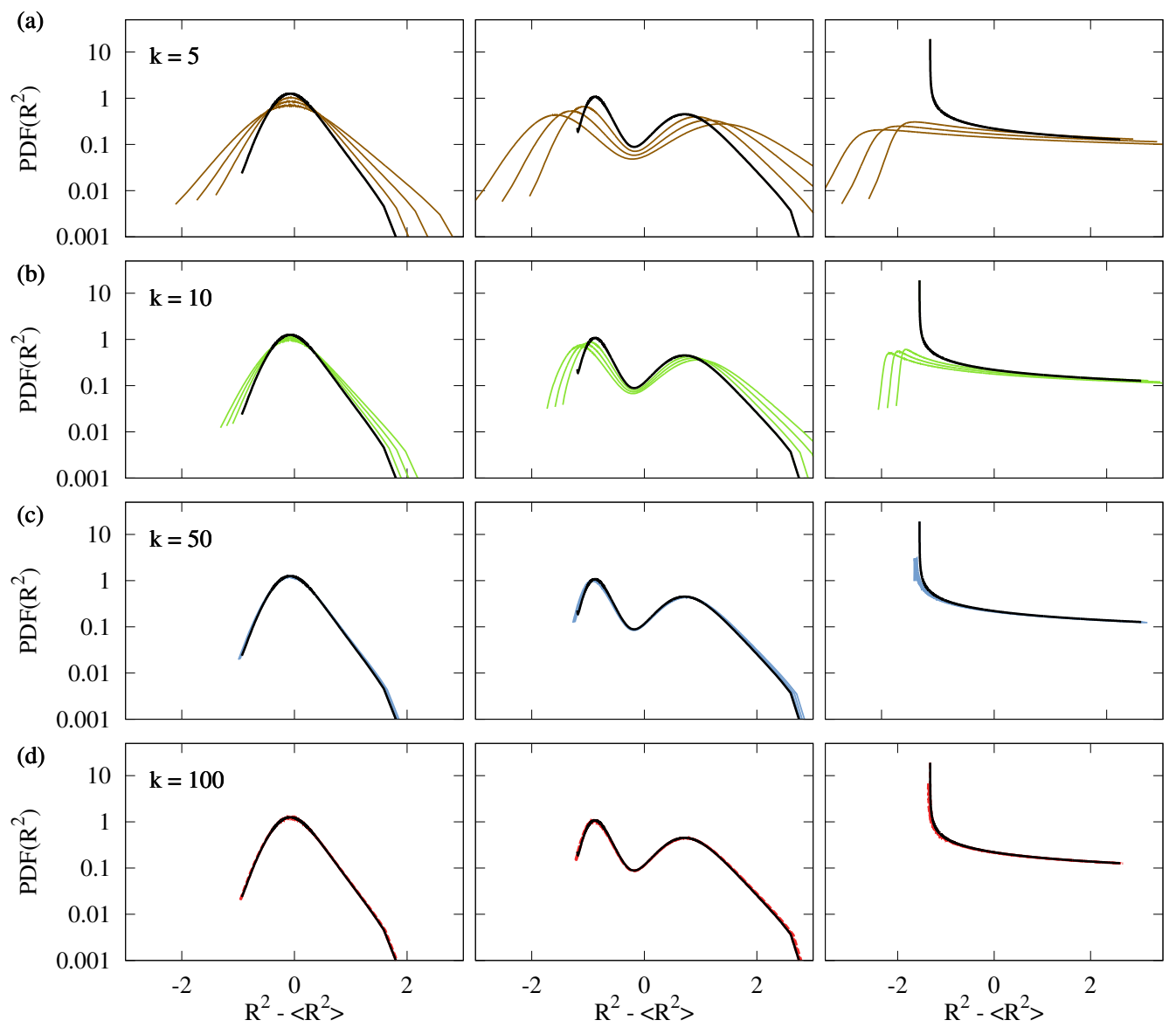

Figure 4.3: Evolution of the PDF for initial $k$ values of (a) $k=5$, (b) $k=10$, (c) $k=50$ and (d) $k=100$, respectively. The left panels show the evolution for the bell-shaped, the middle for the bimodal and the right panels for the uniform initial distribution (black lines). The coloured lines show the distributions for time increments, where the overall volume is increased by a factor of 10 between subsequent lines. The distributions are shown as a function of $R^{2}-\left\langle R^{2}\right\rangle$ such that $\left\langle R_{0}^{2}\right\rangle=1.03$ for the bell-shaped, 1.19 for the bimodal and 1.33 for the uniform distribution, respectively. 
the aggregate assembly which is derived in section 1.4

$$
\dot{R}_{i}=\frac{D \sigma}{R_{i}^{2}}\left(k \frac{R_{i}}{\langle R\rangle}-1\right) \quad \text { with } \quad k=1+\frac{\xi \mathcal{V}}{4 \pi D \sigma N} .
$$

Remarkably it is possible to describe also the dynamics at late times for values of about $k \approx 5$.

The discussion is based on our paper Vollmer et al. (2014). We will start this section by a close look at the moments of the aggregate size distribution, followed by the analysis of the evolution of the reduced radius $R /\langle R\rangle$. Finally we describe the evolution of the size distribution for the uniform initial distribution. The theory is compared to the evolution of the uniform initial distribution.

\subsubsection{Moments of the aggregate size distribution}

To gain insight into the long-time behaviour of the evolution of an assembly of aggregates it is instructive to discuss the time evolution of the cumulants of the size distribution. Doing so we will assume that the number of aggregates, $N$, remains constant in the evolution. This has been reported by Sugimoto (1992); Tokuyama and Enomoto (1993); Clark et al. (2011) and we further discuss the change in aggregate number in section 4.2.2.1.

\subsubsection{The evolution of $\left\langle R^{3}\right\rangle$}

By averaging eq. (4.6) we gain the mean volume growth rate of the assembly

$$
\frac{\mathrm{d}}{\mathrm{d} t}\left\langle R^{3}\right\rangle=3 D \sigma(k-1)
$$

that is indeed constant for a constant $k$ value. Integration leads to

$$
\left\langle R^{3}\right\rangle=\frac{3 V}{4 \pi N}=3 \sigma D(k-1)\left(t-t_{0}\right),
$$

where $t_{0}$ denotes the time, when the initial volume has doubled.

\subsubsection{The average aggregate radius $\langle R\rangle$}

For a constant number of particles the time derivative of the average aggregate radius

$$
\langle R\rangle=\frac{1}{N} \sum_{i} R_{i},
$$




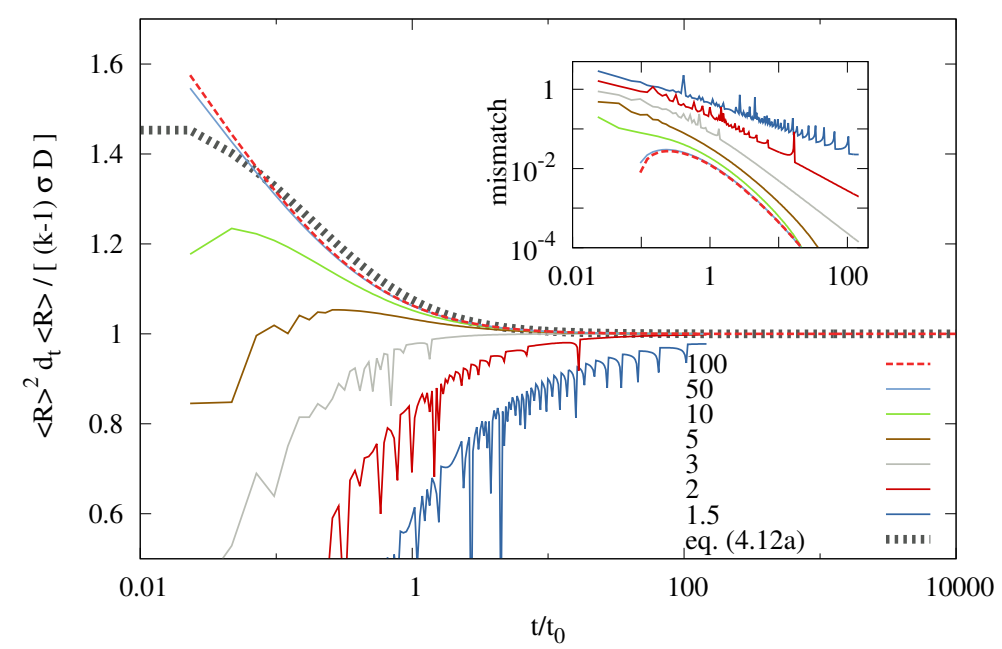

Figure 4.4: Evolution of $\langle R\rangle^{2} \frac{\mathrm{d}}{\mathrm{d} t}\langle R\rangle$ for different values of $k$, as indicated in the legend. When, for $k \gtrsim 5$ the average aggregate volume has increased by a factor of two (i.e. $t=2 t_{0}$ ) one may safely assume that $\langle R\rangle^{2} \frac{\mathrm{d}}{\mathrm{d} t}\langle R\rangle=\sigma D(k-1)$ as predicted by eq. (4.9). The inset shows the mismatch of the numerical data and the improved prediction, eq. (4.12a).

based on eq. (4.6) is given by

$$
\begin{aligned}
\Rightarrow \quad \frac{\mathrm{d}}{\mathrm{d} t}\langle R\rangle & =\frac{1}{N} \sum_{i} \dot{R}_{i}=\frac{1}{N} \sum_{i} \frac{\sigma D}{R_{i}^{2}}\left[k \frac{R_{i}}{\langle R\rangle}-1\right] \\
& =\frac{\sigma D}{\langle R\rangle^{2}}\left[k\left\langle R^{-1}\right\rangle\langle R\rangle-\left\langle R^{-2}\right\rangle\langle R\rangle^{2}\right] .
\end{aligned}
$$

Since the size distribution becomes monodisperse in the long-time limit the products $\left\langle R^{-1}\right\rangle\langle R\rangle$ and $\left\langle R^{-2}\right\rangle\langle R\rangle^{2}$ eventually approaches one. In this limit the characteristic aggregate volume, $(4 \pi / 3)\langle R\rangle^{3}$, follows exactly the same law, eq. (4.8), as the growth of the average aggregate volume $(4 \pi / 3)\left\langle R^{3}\right\rangle$,

$$
\langle R\rangle^{2} \frac{\mathrm{d}}{\mathrm{d} t}\langle R\rangle=\sigma D(k-1) \text { for large } t .
$$

This is demonstrated in fig. 4.4 by showing that the ratio $\langle R\rangle^{2} \frac{\mathrm{d}}{\mathrm{d} t}\langle R\rangle /[\sigma D(k-1)]$ settles to one after some initial transient. 


\subsubsection{Deviation of $\langle R\rangle^{3}$ from $\left\langle R^{3}\right\rangle$}

Equations (4.8) and (4.9) state that in the long run the expectation values $\langle R\rangle^{3}$ from $\left\langle R^{3}\right\rangle$ acquire the same slope as functions of time. In order to also gain insight into the difference of the two functions, we first consider the expectation value $\left\langle R^{4}\right\rangle$.

We use $R=\langle R\rangle+(R-\langle R\rangle)$ and the forth power of this expression to observe that

$$
\begin{aligned}
\left\langle R^{4}\right\rangle-\left\langle R^{2}\right\rangle^{2} & =-\left(\left\langle R^{2}\right\rangle+\langle R\rangle^{2}\right)\left\langle(R-\langle R\rangle)^{2}\right\rangle \\
& +6\langle R\rangle^{2}\left\langle(R-\langle R\rangle)^{2}\right\rangle \\
& +4\langle R\rangle\left\langle(R-\langle R\rangle)^{3}\right\rangle \\
& +\left\langle(R-\langle R\rangle)^{4}\right\rangle \\
& \simeq 4\langle R\rangle^{2}\left\langle(R-\langle R\rangle)^{2}\right\rangle .
\end{aligned}
$$

Here we neglected the cubic term $4\langle R\rangle\left\langle(R-\langle R\rangle)^{3}\right\rangle$ as well as the quartic terms $\left\langle(R-\langle R\rangle)^{4}\right\rangle$ and $\left\langle(R-\langle R\rangle)^{2}\right\rangle^{2}$. These expectation values are sub-dominant contributions for a sharp distribution, where $(R-\langle R\rangle) \ll R$ for the vast majority of aggregates. (Asymptotically, they are smaller by a factor of order $\langle R\rangle^{-2}$.)

The result, eq. (4.10) provides an important insight into the leading order contribution to $\left\langle R^{3}\right\rangle-\langle R\rangle^{3}$,

$$
\begin{aligned}
\left\langle R^{3}\right\rangle & =\left\langle[\langle R\rangle+(R-\langle R\rangle)]^{3}\right\rangle \\
& \simeq\langle R\rangle^{3}+3\langle R\rangle\left\langle(R-\langle R\rangle)^{2}\right\rangle \\
& \simeq\langle R\rangle^{3}+\frac{3}{4\langle R\rangle}\left\langle\left(R^{2}-\left\langle R^{2}\right\rangle\right)^{2}\right\rangle
\end{aligned}
$$

where we used eq. (4.10) in the last step. Rearranging the equation we find

$$
\begin{aligned}
\left\langle R^{3}\right\rangle-\langle R\rangle^{3} & =\frac{3 \Omega_{2}}{4\langle R\rangle} \\
\text { with } \quad \Omega_{2} & =\left\langle\left(R^{2}-\left\langle R^{2}\right\rangle\right)^{2}\right\rangle .
\end{aligned}
$$

The inset of fig. 4.4 shows that for $t \gg t_{0}$ the leading order correction to eq. (4.9) decays like $t^{-4 / 3}$. This amounts to the time dependence of the derivative of $\langle R\rangle^{-1} \sim$ $t^{-1 / 3}$. In view of eq. (4.11a) this suggests that $\Omega_{2}$ approaches a constant value to a very good approximation. 
Making use of this finding the time derivative of eq. (4.11a) can be determined straight-forwardly, yielding

$$
\begin{aligned}
\langle R\rangle^{2} \frac{\mathrm{d}}{\mathrm{d} t}\langle R\rangle & =\sigma D(k-1)+\frac{\Omega_{2}}{4\langle R\rangle^{4}}\langle R\rangle^{2} \frac{\mathrm{d}}{\mathrm{d} t}\langle R\rangle \\
\Leftrightarrow\langle R\rangle^{2} \frac{\mathrm{d}}{\mathrm{d} t}\langle R\rangle & =\sigma D(k-1)\left(1-\frac{\Omega_{2}}{4\langle R\rangle^{4}}\right)^{-1} .
\end{aligned}
$$

The thick dotted line in the main panel of fig. 4.4 shows the resulting prediction for the leading order corrections to eq. (4.9) when one assumes that $\Omega_{2}$ never noticeably deviates from its initial value

$$
\Omega_{2}=\frac{1}{5} \frac{R_{\max }^{5}-R_{\min }^{5}}{R_{\max }-R_{\min }}-\left(\frac{1}{3} \frac{R_{\max }^{3}-R_{\min }^{3}}{R_{\max }-R_{\min }}\right)^{2}
$$

determined for the uniform initial aggregate size distribution, eq. (3.15). For the specified values $R_{\max }=3$ and $R_{\min }=0.02$ it takes the value $\Omega_{2} \simeq 7.19$. This parameter-free prediction lies right on top of the data for $k=100$.

\subsubsection{The variance of the distribution}

Equations eq. (4.10) and eq. (4.11b) provide the variance of the aggregate size distribution

$$
\left\langle R^{2}\right\rangle-\langle R\rangle^{2} \simeq \frac{\Omega_{2}}{4\langle R\rangle^{2}}
$$

Remarkably, the standard deviation decays like $\langle R\rangle^{-1}$. Based on the approximation that the aggregate size distribution amounts to a Gaussian at all times this results has previously been obtained by Clark et al. (2011). However, in contrast to eq. (4.13) they have predicted a decay scaling like for the variance $\langle R\rangle^{-2+2 /(k-1)}$. In section 4.2.3.2 we will show that this discrepancy arises from the fact that $\Omega_{2}$ has a very slight time dependence: it increases like $\langle R\rangle^{1 /(k-1)}$. For large $k$ this correction is negligible such that it was not captures by the present analysis.

The central results of this section are eqs. (4.9) and (4.12a). They express that one can accurately integrate the average radius $\langle R\rangle$ without need to refer to the evolution of the individual aggregates: the average $\langle R\rangle$ need not be calculated self-consistently as an average over the aggregates, but it has its own evolution equation, eq. (4.12a). The solution of this equation explicitly solves the global constraint that couples the set of equations 4.6. This is remarkable because it allows us to reduce the $N$ dimensional system of non-linear coupled equations (4.6) for the aggregate radii, $R_{i}$ into $N$ identical one-dimensional differential equations that only differ by their initial conditions. Henceforth, we concentrate on this equation and suppress the index $i$. 


\subsubsection{Reduced aggregate radius}

In order to gain insight into the evolution of the aggregates we consider the reduced aggregate radius $\rho=R /\langle R\rangle$. Using eq. (4.6) the time derivative of $\rho$ can be written as

$$
\begin{aligned}
\dot{\rho} & =\frac{d}{d t} \frac{R}{\langle R\rangle}=\frac{\dot{R}}{\langle R\rangle}-\rho \frac{\frac{\mathrm{d}}{\mathrm{d} t}\langle R\rangle}{\langle R\rangle} \\
& =\frac{\sigma D}{\langle R\rangle R^{2}}[k \rho-1]-\rho \frac{\frac{\mathrm{d}}{\mathrm{d} t}\langle R\rangle}{\langle R\rangle} \\
& =-\frac{\sigma D}{\langle R\rangle^{3}} \rho^{-2}\left[\frac{\langle R\rangle^{2} \frac{\mathrm{d}}{\mathrm{d} t}\langle R\rangle}{\sigma D} \rho^{3}-k \rho+1\right]
\end{aligned}
$$

According to eq. (4.9) (or fig. 4.4) the factor $\langle R\rangle^{2} \frac{\mathrm{d}}{\mathrm{d} t}\langle R\rangle /(\sigma D)$ approaches $k-1$ after a short initial transient. Consequently,

$$
\begin{aligned}
\dot{\rho} & \simeq-\frac{\sigma D}{\langle R\rangle^{3}} \frac{(k-1) \rho^{3}-k \rho+1}{\rho^{2}} \\
& =-\frac{\sigma D(k-1)}{\langle R\rangle^{3}} \frac{(\rho-1)\left(\rho-\rho_{-}\right)\left(\rho-\rho_{+}\right)}{\rho^{2}}
\end{aligned}
$$

with

$$
\rho_{ \pm}(k)=-\frac{1}{2} \pm \frac{1}{2} \sqrt{\frac{k+3}{k-1}} .
$$

The right-hand side of eq. (4.15a) involves a cubic polynomial in $\rho$ (fig. 4.5). For all $k>1$ it gives rise to three fixed points of the reduced radius: the average aggregate radius $\rho=1$, a non-trivial radius $\rho_{+}$, and an nonphysical fixed point $\rho_{-}$at negative values of $\rho$. Discussing their positions and stability for different reduced temperature ramp rates provides detailed insight into the dynamics.

For $k=1$ we recover classical Ostwald ripening. The radius $\rho_{+}$diverges, and the constraint on the overall aggregate volume gives rise to an asymptotic aggregate size distribution where the largest aggregates are of radius $\rho_{\max }=3 / 2$.

For $1<k<3 / 2$ eq. (4.15a) has an unstable fixed point at $\rho=1$, i.e. for $R=\langle R\rangle$. Aggregates that are smaller than the average radius shrink and they evaporate eventually when they reach the radius $\rho=0$. Aggregates larger than $\rho_{+}$shrink, too, until they reach the stable aggregate radius $\rho_{+}$. On the other hand, aggregates in the range of $1<\rho<\rho_{+}$grow at the expense of the shrinking ones, also striving to reach the aggregate radius $\rho_{+}$. When all aggregates are 


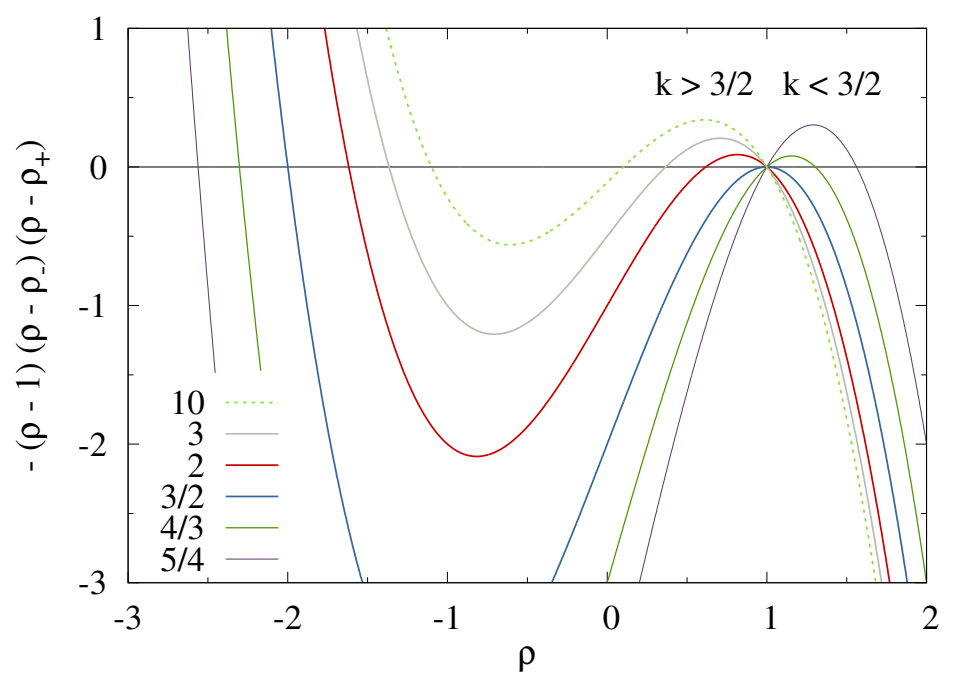

Figure 4.5: The cubic polynomial in the numerator of eq. (4.15a). For all $k>1$ its three roots give rise to three fixed points of the reduced radius, $\rho$ that are located at $\rho=1$ and $\rho=\rho_{ \pm}$. For $k=3 / 2$ there is a bifurcation where the roots $\rho=1$ and $\rho_{+}$change stability.

smaller than $\rho_{+}$and $\rho_{+} \gg 3 / 2$ we expect a similar LSW scaling theory to be applicable as in the Lifshitz-Slyozov-Wagner case $k=1$ (see Slezov (2009) for some pioneering work discussing this situation). In the following we concentrate here on the case $k>3 / 2$, where the LSW theory does not apply.

At $k=3 / 2$ the fixed points $\rho=1$ and $\rho_{+}$cross, and they exchange their stability. Beyond this value aggregate evaporation ceases when all remaining aggregates have a size $\rho>\rho_{+}$.

For $k>3 / 2$ eq. (4.15a) has a stable fixed point for $\rho=1$, and an unstable fixed point at $\rho_{+}$which rapidly approaches $k^{-1}$ for $k \gtrsim 5$. After a brief initial transient no aggregates evaporate any longer, and the distribution becomes strongly peaked around the average aggregate radius $\langle R\rangle$. This is indeed what we have observed in fig. 4.1.

\subsubsection{Evaporation of aggregates}

Aggregates that are smaller than $\langle R\rangle$ by a factor of $\rho_{+}$shrink and evaporate when they reach zero size. For large values of $k$ and reasonably smooth initial aggregate densities this can only be a small fraction of aggregates. Consequently, $n$ does not change much when these aggregates disappear. To support this view we show in fig. 4.6 that to an excellent approximation the number of aggregates bound to 


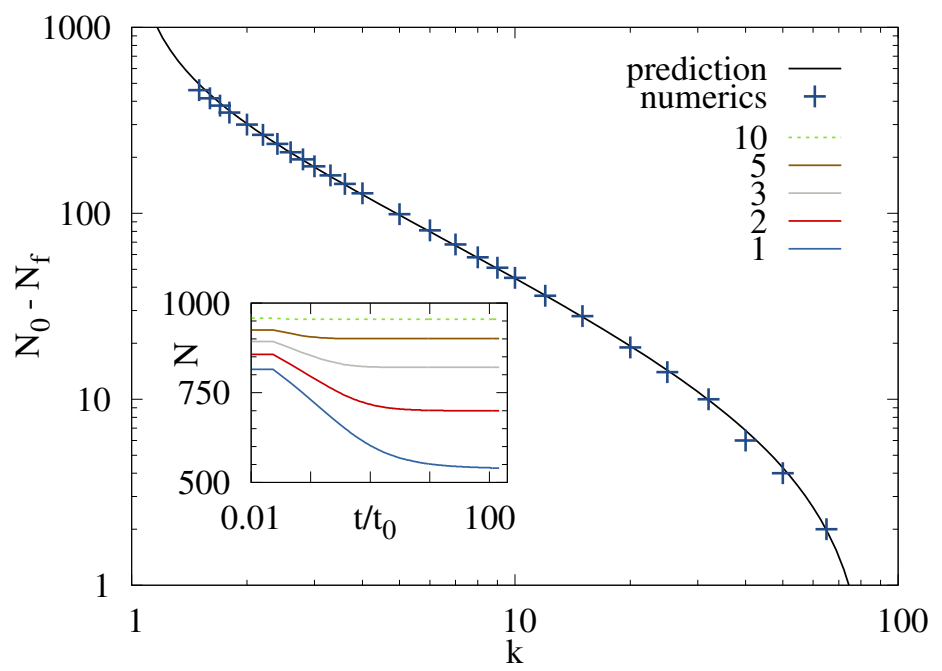

Figure 4.6: Evolution of the aggregate number. The inset shows the time evolution of the number of aggregates for different values of $k$. All systems are initialised with $N_{0}=10^{3}$ aggregates with a uniform size distribution, eq. (3.15). Eventually, they settle down to a constant aggregate number $N_{f}$. The main panel compares the number of evaporated aggregates $N_{0}-N_{f}$ to the prediction that it should amount to $\int_{0}^{\rho_{+}} n\left(\varrho, t=t_{0}\right) \mathrm{d} \varrho$.

evaporate amounts to the number of aggregates in the initial distribution that lie below $\rho_{+}$.

The fate of a general initial distribution for an initial value of $k$ in the range $1<k \leq 3 / 2$ can be discussed based on fig. 4.12. For $1<k \leq 3 / 2$ the aggregates with a radius smaller than average shrink, and eventually they evaporate. While doing so the number density, $n$, decreases. According to eq. (1.9) this results in an increase of $k$. This growth of $k$ continues until all aggregates have a size $\rho>\rho_{+}$, i.e. their size lies above the the red line in fig. 4.12. At that time $k$ takes a value $k \gtrsim 3 / 2$, and in the subsequent long-time limit, $k$ is a constant of motion.

No aggregates should evaporate for $R_{\min } /\langle R\rangle>\rho_{+}\left(k_{c}\right) \simeq k^{-1}$, i.e. for $k_{c}>75$ for the initial conditions specified by eq. (3.15). In practice, the numerical simulations show that the values is slightly smaller. System subjected to a temperature ramp where $k>64$, i.e. for $\xi \gtrsim 250 \pi \sigma D n$ evolves at a constant number density, $n$, of aggregates, and hence at a constant value of $k$. When dealing with numerical data we always indicate the initial value of $k$, and self-consistently take into account its change in the plots. Our focus of attention will be the asymptotics of the shape of the aggregate size distribution. 


\subsubsection{Evolution of the reduced aggregate radius}

For all $k \gtrsim 3 / 2$ and sufficiently late times the evolution of the reduced aggregate radius, $\rho$, can be determined explicitly by integrating eq. (4.14). Introducing the function

$$
a=\langle R\rangle^{3} /[3 \sigma D(k-1)]
$$

and focusing on values $\rho \simeq 1$ we write

$$
\begin{aligned}
3(k-1) a \rho^{2} \dot{\rho} & =-(k-1) \dot{a} \rho^{3}+(k-1) \rho+(\rho-1) \\
& \simeq-(k-1) \rho\left[\dot{a} \rho^{2}-1\right] \\
\Leftrightarrow \quad \frac{2}{3} a^{-1 / 3} & =\frac{\mathrm{d}}{\mathrm{d} t}\left(a^{2 / 3} \rho^{2}\right) .
\end{aligned}
$$

This equation allows us to evaluate the derivative

$$
\begin{aligned}
\frac{\mathrm{d}}{\mathrm{d} t} R^{2} & =[3 \sigma D(k-1)]^{2 / 3} \frac{\mathrm{d}}{\mathrm{d} t}\left(a^{2 / 3} \rho^{2}\right) \\
& =\frac{2 \sigma D(k-1)}{\langle R\rangle}
\end{aligned}
$$

which agrees with the time derivative of $\left\langle R^{2}\right\rangle$ up to a tiny correction

$$
\begin{aligned}
\frac{\mathrm{d}}{\mathrm{d} t}\left\langle R^{2}\right\rangle & =\langle 2 R \dot{R}\rangle \\
& =\frac{2 \sigma D(k-1)}{\langle R\rangle}\left[1+\frac{1-\langle R\rangle\left\langle R^{-1}\right\rangle}{k-1}\right] .
\end{aligned}
$$

Altogether, eqs. (4.18a) and (4.18b) imply that

$$
\frac{\mathrm{d}}{\mathrm{d} t}\left(R^{2}-\left\langle R^{2}\right\rangle\right)=0 .
$$

There can be no merely time-dependent function appearing on the right-hand side of this equation because the expectation value $\left\langle R^{2}-\left\langle R^{2}\right\rangle\right\rangle$ must vanish at any time. The result, eq. (4.19), states that at late times aggregates always grow in such a way that the difference, $R^{2}-\langle R\rangle^{2}$, is preserved. This has immediate implications on the aggregate size distribution which will be discussed in the next section. 


\subsubsection{Evolution of the aggregate size distribution}

According to eq. (3.22) the order of the aggregate radii is preserved by the dynamics: when aggregate $i$ is smaller than aggregate $j$ initially, this holds also at all later times. Based on this observation and the explicit integration of the evolution equation of the aggregate radius, eq. (4.19) one can immediately derive the aggregate size distribution. This is most easily done based on the cumulative aggregate size distribution function $(\mathrm{CDF})$, introduced in section 3.2.

\subsubsection{Initial distribution, and its evolution based on eq. (4.19)}

For convenience of the discussion of the asymptotic shape of the CDF, we immediately remove the aggregates from the initial distribution that will evaporate. According to the arguments underpinned by fig. 4.6 this amounts to the aggregates smaller than $R_{c}=\left\langle R_{0}\right\rangle \rho_{+}(k)$, where $\left\langle R_{0}\right\rangle=\left(R_{\max }+R_{\min }\right) / 2=1.51$ is the average radius with respect to the initial aggregate size distribution eq. (3.15). When no aggregates evaporate we set $R_{c}=R_{\text {min }}$. With this adaption, the CDF characterising the initial distribution, $\mathcal{C}\left(R_{0}\right)$, takes the form

$$
\mathcal{C}\left(R_{0}\right)=\left\{\begin{array}{llc}
0 & \text { for } & R<R_{c} \\
\frac{R-R_{c}}{R_{\max }-R_{c}} & \text { for } & R_{c}<R<R_{\max } \\
1 & \text { for } & R_{\max }<R
\end{array}\right.
$$

To avoid the involved notation required to explicitly distinguish the different branches of the function, we henceforth only specify its non-trivial branch, and keep in mind that the function should be set to zero when the expression drops below zero, and set to one when it rises beyond one.

In order to apply eq. (4.19) it is convenient to rewrite eq. (4.20) as a function of

$$
x=R^{2}-\left\langle R^{2}\right\rangle
$$

In that case the non-trivial dependence for $R_{c}<R<R_{\max }$ takes the form of a square-root dependence

$$
\mathcal{C}(x)=\frac{\left[x+\left\langle R_{0}^{2}\right\rangle\right]^{1 / 2}-R_{c}}{R_{\max }-R_{c}} .
$$

The initial condition $\mathcal{C}(x)$ of the $\mathrm{CDF}$, provided as a function of $x$, is shown by solid black lines in fig. 4.7 .

To determine the time dependence of the CDF we note that according to eq. (4.19) the value of $x$ is preserved during the evolution. Consequently, the CDF should not change in time when it is plotted as a function of $x$. 

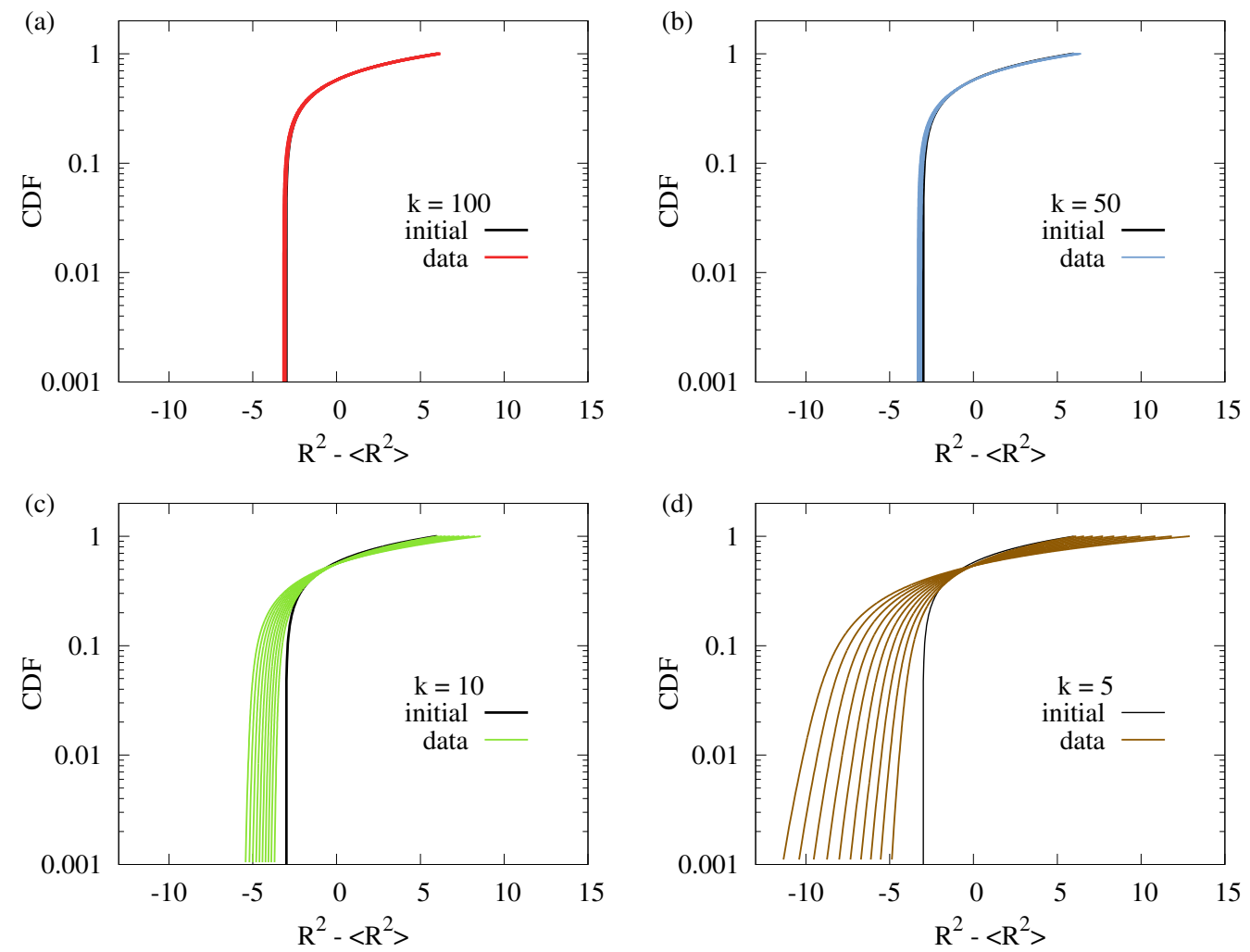

Figure 4.7: The four panels show the CDF of the aggregates for (a) $k=100$, (b) $k=50$, (c) $k=10$, and (d) $k=5$, respectively. The distributions are shown as a function of $x=R^{2}-\left\langle R^{2}\right\rangle$ as suggested in eq. (4.21). The initial conditions are highlighted by thick black lines. The coloured lines show the time evolution for the respective initial $k$ value. This is the same data as in fig. 4.3(right panels) but shown as a CDF.

This description of the CDF properly captures main feature of its time evolution: we observed in fig. 4.3 that the CDFs tend to preserve their form when plotted as a function of $R^{2}-\left\langle R^{2}\right\rangle$. For all $k \gtrsim 50$ this provides an accurate description of the numerical data. On the other hand, for decreasing $k$ the tails of the distributions towards the smaller aggregate sizes tend to become less steep, and in addition there is a noticeable broadening of the distributions in the course of time. These deviations arise from the fact that for $\rho \simeq 1$ we systematically underestimates the slope of $\dot{\rho}$ due to suppressing the term $(\rho-1) /(k-1)$ on the right hand side of eq. (4.17). 


\subsubsection{Accounting for broadening and shape changes}

For late times, where eq. (4.9) applies, we can gain insight into the broadening of the distribution by integrating eq. (4.15) rather than eq. (4.17).

We use eq. (4.16) to write eq. (4.15a) in the form

$$
\dot{\rho}=-\frac{1}{3 a} \frac{(\rho-1)\left(\rho-\rho_{-}\right)\left(\rho-\rho_{+}\right)}{\rho^{2}}
$$

and introduce a function $g(\rho)$ that obeys the differential equation

$$
\frac{\mathrm{d} g}{\mathrm{~d} \rho}=\frac{\rho^{2} g}{(\rho-1)\left(\rho-\rho_{-}\right)\left(\rho-\rho_{+}\right)} .
$$

Combining eqs. (4.23) and (4.24) allows us to rephrase the evolution of $\rho$ in the form

$$
\frac{\dot{g}}{g}=g^{-1} \frac{\mathrm{d} g}{\mathrm{~d} \rho} \dot{\rho}=\frac{-1}{3 a}=-\frac{\dot{a}}{3 a},
$$

where we used in the last step that $\dot{a}=1$ in the long-time asymptotics considered here. Equation 4.25 implies that

$$
\frac{\mathrm{d}}{\mathrm{d} t}\left(g a^{1 / 3}\right)=0 .
$$

In order to interpret this finding we have to find the function $g$. The differential equation eq. (4.24) has solutions of the form

$$
g=C(\rho-1)^{\alpha_{1}}\left(\rho+1+\rho_{+}\right)^{\alpha_{-}}\left(\rho-\rho_{+}\right)^{\alpha_{+}},
$$

where the constant number $C$ represents the integration constant. Inserting eq. (4.27a) into eq. (4.24) provides a linear set of equations for the exponents $\left(\alpha_{1}, \alpha_{-}, \alpha_{+}\right)$that is solved by

$$
\begin{aligned}
& \alpha_{1}=\frac{1}{\left(2+\rho_{+}\right)\left(2+\rho_{-}\right)}=\frac{k-1}{2 k-3}, \\
& \alpha_{-}=\frac{\rho_{-}^{2}}{\left(2+\rho_{+}\right)\left(1+2 \rho_{+}\right)} \simeq \frac{1}{2}-\frac{1}{4 k}+\frac{5}{8 k^{2}}-\ldots, \\
& \alpha_{+}=\frac{\rho_{+}^{2}}{\left(2+\rho_{-}\right)\left(1+2 \rho_{-}\right)} \simeq-\frac{1}{k^{2}}+\ldots
\end{aligned}
$$

Equation 4.26 together with the definition of $a$, eq. (4.16), entails that the cumulative distribution function is a function of $\langle R\rangle g$. Moreover, by comparison to fig. 4.8 

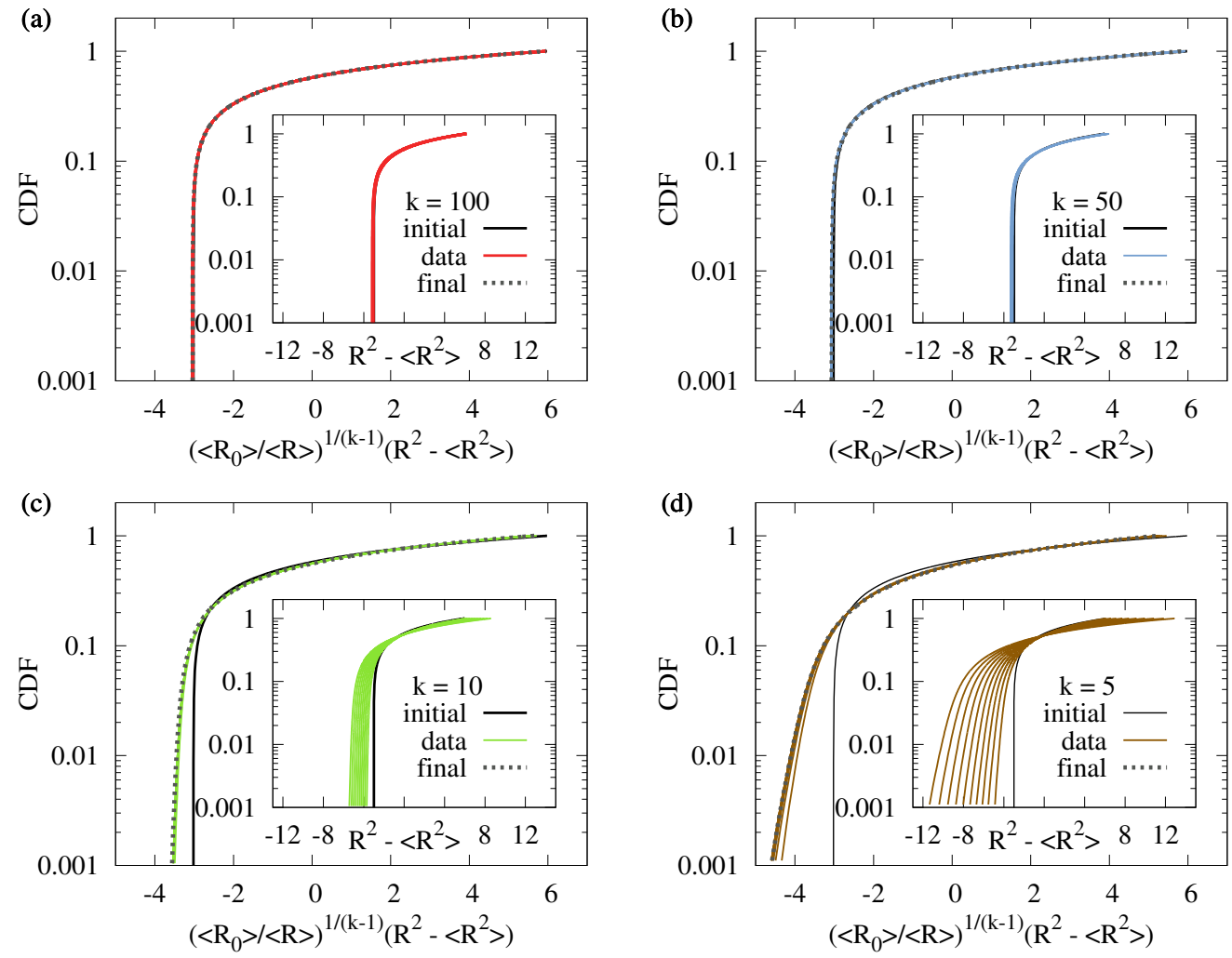

Figure 4.8: The four panels show the CDF of the aggregates for (a) $k=100$, (b) $k=50$, (c) $k=10$, and (d) $k=5$, respectively. The insets show the CDF as a function of $x=R^{2}-\left\langle R^{2}\right\rangle$ as suggested in eq. (4.21), and the main panels the dependence on $\widetilde{x}$ which has been defined in eq. (4.29). The initial conditions are highlighted by thick black lines. The coloured lines show the time evolution for the respective initial $k$ value. As a function of $\widetilde{x}$ they approach an asymptotic distribution ("final").

one finds that in leading order of the long-time asymptotics, where $\left\langle R^{2}\right\rangle=\langle R\rangle^{2}$ (cf. eq. (4.13)), the cumulative distribution function must depend on $R^{2}-\langle R\rangle^{2}=$ $\langle R\rangle^{2}\left(\rho^{2}-1\right)$. This dependence can be faithfully recovered from $(\langle R\rangle g)^{1 / \alpha_{1}}$ by observing that $\alpha_{1}^{-1}=2-(k-1)^{-1}$. Moreover, making use of $\alpha_{1}+\alpha_{+}+\alpha_{-}=1$ one 
easily shows that $\alpha_{-} / \alpha_{1}=1-(k-1)^{-1}-\alpha_{+} / \alpha_{1}$. These relations provide

$$
\begin{aligned}
&(\langle R\rangle g)^{\alpha_{1}^{-1}=}\langle R\rangle^{2-(k-1)^{-1}}\left(\rho^{2}-1\right)\left(1+\frac{\rho_{+}}{\rho+1}\right) \\
&\left(\rho+1+\rho_{+}\right)^{-(k-1)^{-1}}\left(\frac{\rho-\rho_{+}}{\rho+1+\rho_{+}}\right)^{\alpha_{+} / \alpha_{1}} \\
& \simeq\langle R\rangle^{-(k-1)^{-1}}\left(R^{2}-\langle R\rangle^{2}\right)\left[1+\mathcal{O}\left((k-1)^{-1}\right)\right] .
\end{aligned}
$$

In line with our interpretation of the data shown in the insets of fig. 4.8 equation eq. (4.28b) predicts a sustained broadening of the distribution via the factor $\langle R\rangle^{-1 /(k-1)}$ that leads to increasingly pronounced effects for smaller values of $k$. Moreover, for not too large values of $k$ the higher-order corrections specified by the last three factors in eq. (4.28a) affect the relation between $R^{2}-\left\langle R^{2}\right\rangle$ and its initial value $R_{0}^{2}-\left\langle R_{0}^{2}\right\rangle$ such that the shape of the distribution need not longer be preserved. The slowly expanding variable

$$
\widetilde{x}=\left(\frac{\left\langle R_{0}\right\rangle}{\langle R\rangle}\right)^{(k-1)^{-1}}\left(R^{2}-\left\langle R^{2}\right\rangle\right)
$$

accounts for the sustained broadening of the CDF via the factor $\langle R\rangle^{-(k-1)^{-1}}$, and at early times it appropriately fixes the mean position of the CDF, as observed in eq. (4.21).

Except for the early times for the cases $k \leq 10$ the cumulative distribution function eq. (4.22) as a function of the argument $\widetilde{x}$ provides an accurate description of distributions at all times. This is demonstrated by the data collapse shown in the main panels of fig. 4.8. In particular, $\widetilde{x}$ faithfully accounts for the broadening of the distribution that was severely underestimated previously. Moreover, the factors in eq. (4.28a) that have been suppressed in the choice eq. (4.29) account for the change of the shape of the distribution: the dashed black lines show the shape of the distribution that results when these factors are accounted for.

\subsubsection{Scaling of the centred moments of the size distribution}

The observation that the aggregate size distribution is invariant when plotted as a function of $\widetilde{x}$ has immediate consequences for the centred moments of the size distribution function. First of all it implies that $\left\langle\widetilde{x}^{n}\right\rangle$ is invariant in time such that

$$
\Omega_{n}:=\left\langle\left(R^{2}-\left\langle R^{2}\right\rangle\right)^{n}\right\rangle \sim\left(\frac{\langle R\rangle}{\left\langle R_{0}\right\rangle}\right)^{n /(k-1)}
$$


For small $k$ the factor $\langle R\rangle^{2 /(k-1)}$ provides a small, but noticeable growth of $\Omega_{2}$ that is reflected in the broadening of the distributions shown in the insets of fig. 4.8. In order to calculate the centred moments we note that

$$
\begin{aligned}
R-\langle R\rangle & =\frac{\left(R^{2}-\left\langle R^{2}\right\rangle\right)-(R-\langle R\rangle)^{2}+\left\langle(R-\langle R\rangle)^{2}\right\rangle}{2\langle R\rangle} \\
& =\frac{1}{2\langle R\rangle}\left[\left(R^{2}-\left\langle R^{2}\right\rangle\right)+\mathcal{O}\left(\langle R\rangle^{-2}\right)\right]
\end{aligned}
$$

Consequently,

$$
\left\langle(R-\langle R\rangle)^{n}\right\rangle \simeq\left\langle\left(\frac{R^{2}-\left\langle R^{2}\right\rangle}{2\langle R\rangle}\right)^{n}\right\rangle=\frac{\Omega_{n}}{(2\langle R\rangle)^{n}}
$$

In view of the asymptotic scaling, eq. (4.30), of $\Omega_{n}$ this implies

$$
\left\langle(R-\langle R\rangle)^{n}\right\rangle \sim\langle R\rangle^{-n+n /(k-1)} .
$$

In particular, we hence obtain the result anticipated in section 4.2.1.4: the standard deviation of the aggregate size distribution decays like

$$
\sqrt{\left\langle R^{2}\right\rangle-\langle R\rangle^{2}}=\frac{\Omega_{2}{ }^{1 / 2}}{2\langle R\rangle} \sim\langle R\rangle^{-1+(k-1)^{-1}} .
$$

\subsection{Size distributions in the experiment}

This section deals with the measured size distributions of droplets in binary phase separation. What is the impact of droplets which are too large to be considered as Brownian particles anymore and move under the influence of gravity? To disentangle the contribution of droplet growth coming from a constant ramp rate $\xi$ and the effects of sedimentation, we performed jump experiments: droplets are grown with a constant ramp rate, and when they are large enough to sediment the driving is turned off, i.e. the ramp rate jumps to $\xi=0$. The temperature is kept constant for the remaining time of the measurement.

The measurements are conducted in the lower layer of IBE+W mixtures, with 0.26 mass fraction of IBE. The size distribution of the droplets are determined with the setup and procedure described in section 2.5. The droplets are grown with a ramp rate of $\xi=2.5 \times 10^{-5} \mathrm{~s}^{-1}$ before the jump. The temperature ramps are calculated for a starting temperature of $26^{\circ} \mathrm{C}$. The experiments are done in collaboration with Marcel Ernst. I use here the same data initially described in his Bachelor's thesis (Ernst, 2014), but base my analysis on a new interpretation of the time where the 
size distribution becomes bimodal.

In the first part of the present section I describe the evolution of the measured size distribution. Subsequently I address the impact of sedimentation on the evolution of the size distribution for vanishing ramp rate. Finally the experimental data is compared to the model.

\subsubsection{Experimental observations}

In fig. 4.9(a) the time evolution of the size distribution is shown for a measurement where the ramp rate jumps to $\xi=0$ after $2000 \mathrm{~s}$, as indicated by the vertical red line. After a short offset more and more droplets become visible. They grow in size and their distribution becomes broader. After the jump the number of large droplets decreases and the distribution becomes smaller again. In the end a small amount of droplets is left with a radius of about $10 \mu \mathrm{m}$. To gain insight into this evolution the number density per radius $n(r, t)$ is given for equidistant times before (fig. 4.9(b)) and after (fig. 4.9(c)) the jump. The given size distributions in fig. 4.9(b) and fig. 4.9(c) correspond to the temporal average over three time bins in fig. 4.9(a). Before the jump a peak at around $10 \mu \mathrm{m}$ develops, it grows and shifts slightly to the right. This shift is due to the growth of the droplets in the assembly. However, also the number density ${ }^{1} n(t)$ increases as the peak develops. In the previous section we have seen that the number density of a growing assembly should be constant for large values of $k$ and that is decays for small values. The maximum value $n(t)$ in fig. 4.11 corresponds to a number density $n=10^{12} \mathrm{~m}^{-3}$. Together with the material constants for $27^{\circ} \mathrm{C}$, that are provided in the appendix, this amounts to a value of $k=1.5 \times 10^{6}$. Hence, we expect that the assembly evolves with a constant number density. The radius of the smallest droplets in the size distributions is $5 \mu \mathrm{m}$. This amounts to the radius of the droplets, which are reliably detectable in the measurement. Therefore, it is very likely that there are droplets with a smaller radius and that they become visible in the size distributions only when their radius has grown to a size larger than $5 \mu \mathrm{m}$. Hence, as time goes on, more and more droplets are considered in the size distribution and the number of droplets seems to increase. To understand this increase, although we expect a constant number density, we plot the apparent size distribution, which corresponds to the size distributions measured in the experiment, in fig. 4.9(d) by the dashed blue line. This apparent size distribution can be interpreted as a product of the size distribution of the assembly of growing droplets with a constant number density (red lines in fig. 4.9(d)), and a function which describes the radius dependent probability of the droplet detection (green line in fig. 4.9(d)).

\footnotetext{
${ }^{1}$ The number density $n(t)=\int n(r, t) \mathrm{d} r$ described the number of droplets per unit volume. It corresponds to the area under the $n(r, t)$ curves shown in fig. $4.9(\mathrm{~b}, \mathrm{c})$. Be aware of the logarithmic density axis in those plots.
} 

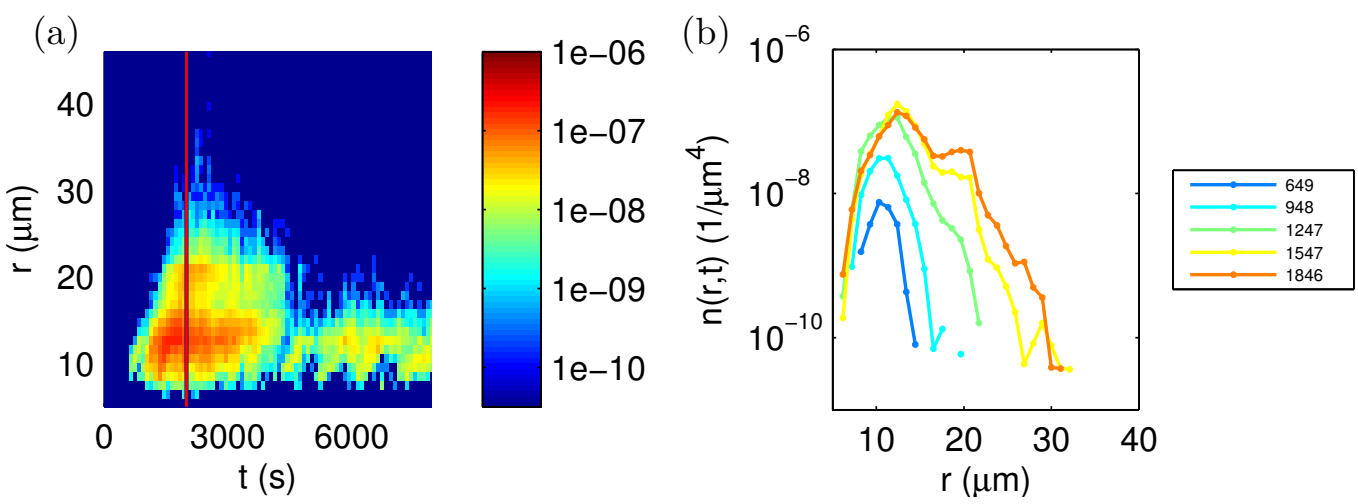

(d)
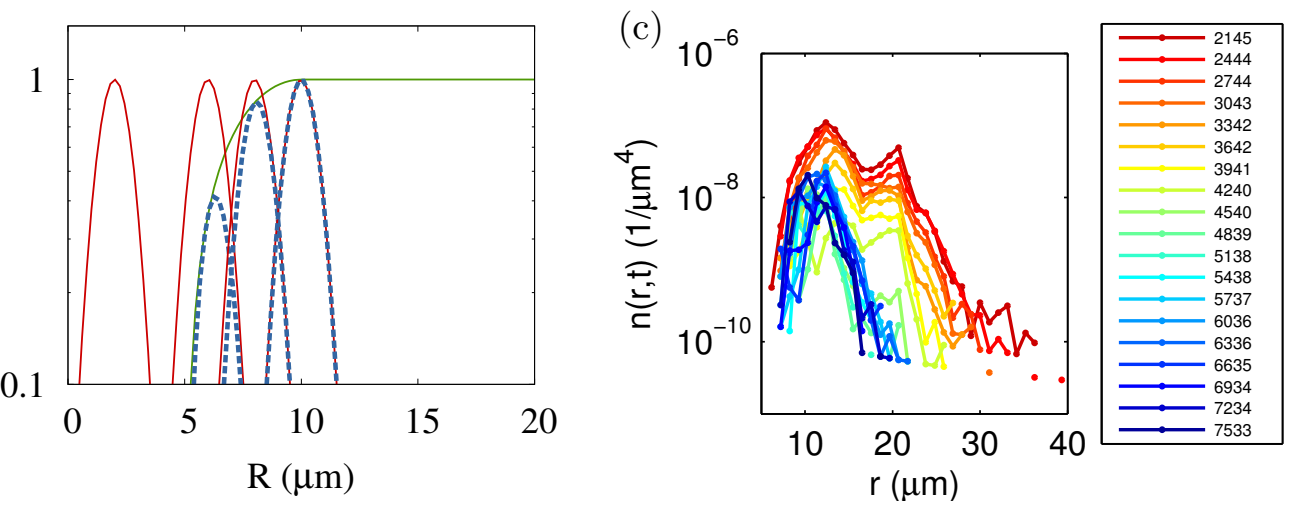

Figure 4.9: (a) Experimental data of the time evolution of the size distribution. The number density per radius is colour coded using a logarithmic scale and given in units of $\mu \mathrm{m}^{-4}$. The red vertical line at $2000 \mathrm{~s}$ indicates the jump of the ramp rate to $\xi=0$. Panels (b) and (c) show the number density per radius for equidistant times (see legends where time is provided in seconds) before and after the jump, respectively. Here time is colour coded: from blue in the beginning to red at the time of the jump, and then in panel (c) back from red to blue. (d) Sketch of the observed size distribution (dashed blue) as a product of the assembly distribution (solid red) and the probability of droplet detection (solid green). Pairs of red and blue lines show the actual (red) and observed (dashed blue) distributions at different times, where the mean size moves to larger radii. The sketch motivates the rise in apparent number density observed in the first three curves in panel (b). 
Let us now return to the interpretation of the data shown in fig. 4.9(b). Around $500 \mathrm{~s}$ before the jump (yellow line for $1547 \mathrm{~s}$ ) a second peak at about $20 \mu \mathrm{m}$ starts to develop. Shortly after the jump (the line at $2444 \mathrm{~s}$ ) the droplet number density decreases and eventually there only remain droplets for sizes around $10 \mu \mathrm{m}$ (blue lines in fig. 4.9(c)).

I attribute the rise of the second peak to the appearance of droplets which are large enough to be affected by gravity. They have considerable settling velocities and can also grow by collecting smaller droplets in their way through the sample. After the jump both peaks of the bimodal size distribution decrease. The peak of the smaller droplets decays, because they are collected by the large sedimenting droplets. The peak of the large droplets decays, because they leave the measurement volume.

In conclusion, we can follow in fig. 4.9 the growth of an assembly of droplets. However, a detailed comparison with the predictions from the theory for large $k$ values, section 4.2 , is difficult, because the assembly can be tracked only over a short range of radii, where the droplet motion is not yet affected by gravity. The optical detection of droplets is bounded at the lower side at about $5 \mu \mathrm{m}$, and the peak of $20 \mu \mathrm{m}$ is identified with the effects of sedimenting droplets. Hence, I investigate in the following the evolution of the size distribution, where the droplets settle in the gravitational field and grow by collection of smaller droplets.

\subsubsection{Growth law for sedimenting droplets}

When the droplets are large enough, i.e. when the motion induced by the density difference between the bulk fluid and the droplet becomes larger than the Brownian motion, they start to sediment. Since the Reynolds number, based on the settling velocity and the diameter of the droplets, is much smaller than one, the droplets move with the Stokes settling velocity. This velocity is the result of a force balance between buoyancy and Stokes drag. According to Stokes' formula the velocity of a slowly settling droplet is (Taylor and Acrivos, 1964; Guyon et al., 2001)

$$
u=\kappa r^{2} \quad \text { with } \quad \kappa=\frac{2}{9} \frac{g \Delta \rho}{\mu_{b}} \frac{\mu_{d}+\mu_{b}}{\mu_{d}+\frac{2}{3} \mu_{b}},
$$

where $g$ is the gravitational acceleration, $\Delta \rho$ the density contrast, $\mu_{b}$ is the dynamic viscosity of the bulk phase, and $\mu_{d}$ is the viscosity of the material in the droplets. For rigid spheres one has $\mu_{d} \gg \mu_{b}$ such that the latter factor in eq. (4.33) takes a value of one.

With the sedimentation velocity $u$ a growth law is obtained for large droplets growing by collection of smaller droplets, while moving their way through the fluid. The volume growth rate of a droplet is proportional to its geometrical cross section $\pi r^{2}$ and the settling velocity $\kappa r^{2}$. Here the approximation is made, that the settling 
velocity of the smaller droplets is negligible. To a good approximation they are still Brownian particles when they are collected by the larger droplets. The small droplets which are in the path of the big droplet, are not taken into account individually but are assumed to be present with a volume fraction $\phi_{0}$. Putting all parts together, the volume growth rate of a large droplet growing by collecting smaller droplets is

$$
4 \pi r^{2} \frac{\mathrm{d} r}{\mathrm{~d} t}=\pi r^{2} \kappa r^{2} \phi_{0} \quad \Leftrightarrow \quad \frac{\mathrm{d} r}{\mathrm{~d} t}=\frac{\kappa r^{2} \phi_{0}}{4} .
$$

\subsubsection{Evolution of the size distribution with sedimentation}

To implement the sedimentation of droplets in the size distribution, $n(r, t)$, the droplet flux of sedimenting droplets is added to the continuity equation of the size distribution. The loss of droplets due to sedimentation happens with a radius dependent rate of $\kappa r^{2} / L$ which is the ratio of the sedimentation speed and the system size $L$. Adding the contribution of the sedimentation to the continuity equation, eq. (1.7), leads to

$$
\frac{\partial n}{\partial t}=-\frac{\partial}{\partial r}\left(\frac{\mathrm{d} r}{\mathrm{~d} t} n\right)-\frac{\kappa r^{2}}{L} n .
$$

In this equation the term $\frac{\mathrm{d} r}{\mathrm{~d} t}$ in the brackets refers to the growth law of the droplets, eq. (4.34). Together with the dimensionless units

$$
x=\frac{r}{L} \quad \text { and } \quad \tau=\frac{t}{T}=t \kappa L
$$

I get the following dimensionless form of eq. (4.35) with $\widetilde{n}=n L^{4}$

$$
\frac{\partial \widetilde{n}}{\partial \tau}=-\frac{\phi_{0}}{4} \frac{\partial}{\partial x}\left(x^{2} \widetilde{n}\right)-x^{2} \widetilde{n} .
$$

for a constant volume fraction of small droplets $\phi_{0}$ eq. (4.37) can be solved by a separation ansatz $\widetilde{n}=g(x) h(\tau)$. This leads to

$$
\frac{1}{h} \frac{\mathrm{d} h}{\mathrm{~d} \tau}=-\frac{\phi_{0}}{4 g} \frac{\mathrm{d}}{\mathrm{d} x}\left(x^{2} g\right)-x^{2}=C
$$

with a constant $C$. The time dependent function $h(\tau)$ is easily solved by

$$
h(\tau)=h_{0} \exp (C \tau)
$$

In order to solve the $x$ dependent part I write $g(x)=\frac{1}{x^{2}} e^{f(x)}$, and get a differential equation for $f(x)$

$$
\frac{\mathrm{d} f}{\mathrm{~d} x}=-\frac{4}{\phi_{0}}\left(1+\frac{C}{x^{2}}\right)
$$


which is solved by

$$
f(x)=-\frac{4 x}{\phi_{0}}+\frac{4 C}{\phi_{0} x} .
$$

Hence, the solution for the radius dependent part is

$$
\begin{aligned}
g(x) & =\frac{g_{0}}{x^{2}} \exp \left(-\frac{4 x}{\phi_{0}}+\frac{4 C}{\phi_{0} x}\right) \\
\Rightarrow \widetilde{n}(x, \tau) & =\widetilde{n}_{0} \frac{e^{C \tau}}{x^{2}} \exp \left[\frac{4}{\phi_{0}}\left(\frac{C}{x}-x\right)\right]
\end{aligned}
$$

To determine the integration constants $\widetilde{n}_{0}$ and $C$ the total number density $\widetilde{n}(\tau)$ is determined

$$
\widetilde{n}(\tau)=\int_{x_{\text {min }}}^{\infty} \widetilde{n}(x, \tau) \mathrm{d} x=\widetilde{n}_{0} e^{C \tau} \int_{x_{\text {min }}}^{\infty} \frac{1}{x^{2}} \exp \left[\frac{4}{\phi_{0}}\left(\frac{C}{x}-x\right)\right] \mathrm{d} x .
$$

For $x_{\text {min }}>0$ the integral has a finite value. Solving eq. (4.44) for the term $\widetilde{n}_{0} e^{C \tau}$ and substituting it in eq. (4.43) leads to

$$
\Rightarrow \widetilde{n}(x, \tau)=\frac{\widetilde{n}(\tau)}{x^{2}} \exp \left[\frac{4}{\phi_{0}}\left(\frac{C}{x}-x\right)\right]\left(\int_{x_{m i n}}^{\infty} \frac{1}{x^{2}} \exp \left[\frac{4}{\phi_{0}}\left(\frac{C}{x}-x\right)\right] \mathrm{d} x\right)^{-1} .
$$

With eq. (4.36) the solution can also be written in a non-dimensionless form with

$$
\begin{gathered}
a=-\kappa L C \text { and } r_{n}=\left(\int_{r_{\text {min }}}^{\infty} \frac{1}{r^{2}} \exp \left(-\frac{4 r}{\phi_{0} L}-\frac{4 a}{\phi_{0} \kappa r}\right) \mathrm{d} x\right)^{-1} \\
n(r, t)=\frac{n(t) r_{n}}{r^{2}} \exp \left(-\frac{4 r}{\phi_{0} L}-\frac{4 a}{\phi_{0} \kappa r}\right) \\
n(t)=n_{0} e^{-a t}
\end{gathered}
$$

where $n_{0}$ is the total number density for $t=0$.

\subsubsection{Comparison with theory}

In this section I compare the measured size distributions (fig. 4.9) to the solution for the number density $n(r, t)$ in eq. (4.46). On the one hand eq. (4.46) predicts a collapse of the distributions for different times, when they are rescaled with $r^{2} / n(t)$. Indeed the size distribution collapse, see fig. 4.10(c)-(d). The red and orange curves lie on top of each other. They correspond to distributions at times where the distribution is bimodal and large sedimenting droplets are present. Distinct from that population, also the blue and greenish curves collapse and form the peak of the Brownian particles. 

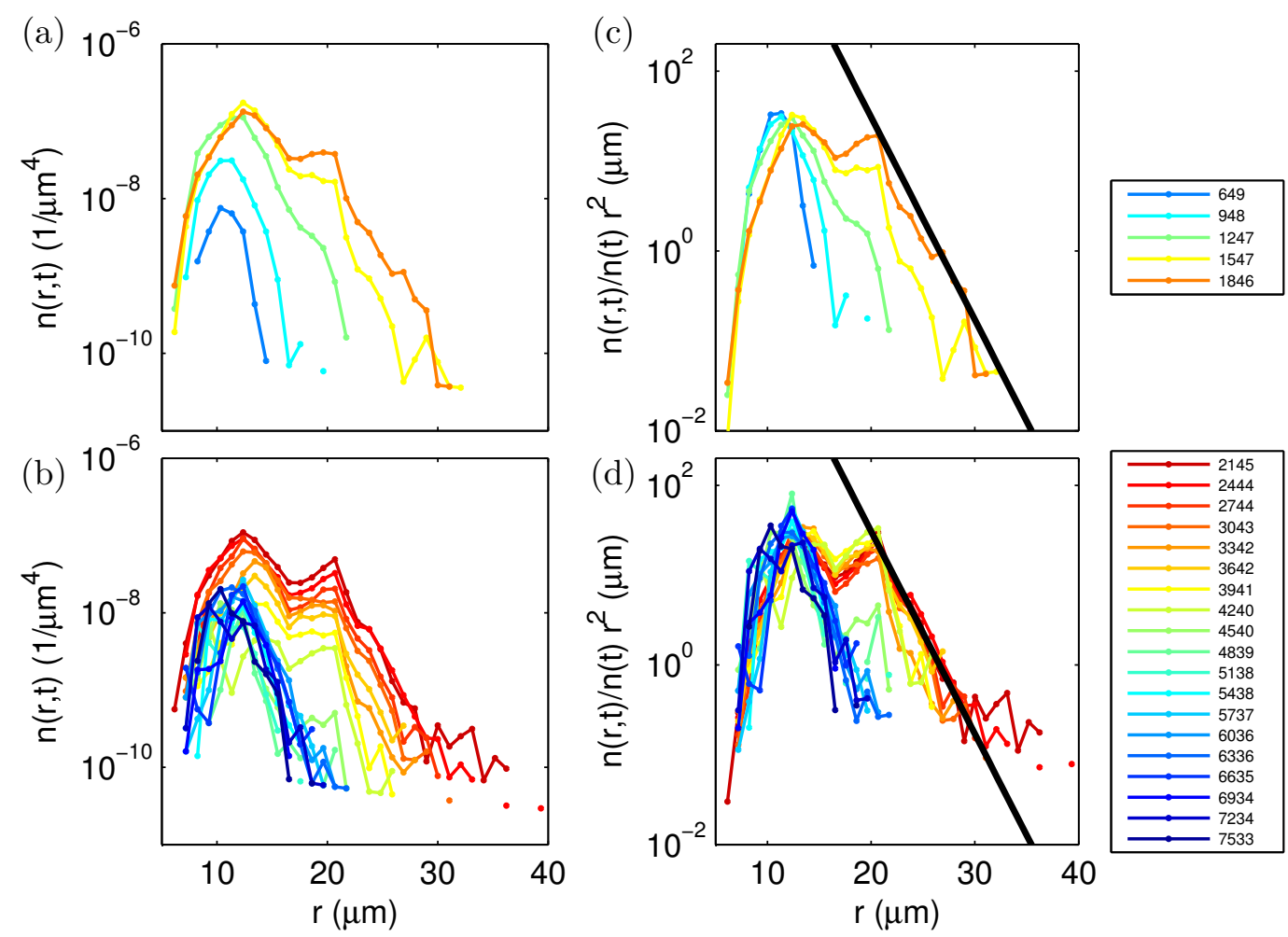

Figure 4.10: Evolution of the number density $n(r, t)$ as a function of radius (a) before and (b) after the jump, respectively, and rescaled number density according to eq. (4.46) (c) before and (d) after the jump, respectively. The time is indicated in the legend, it changes from blue to red at the jump back to blue. The solid black line shows the decaying exponential function $\exp (-4 r / 7.7 \mu \mathrm{m}) \mathrm{m}$ that is obtained by a fit of the data in the intermediate part (red and yellow curves) for radii larger than $r_{p} \approx 20 \mu \mathrm{m}$.

On the other hand the solution for the number density $n(r, t)$, eq. (4.46), depends on several parameters: the decay rate $a$ for the number density $n(t)$, the length scale of the system $L$, the volume fraction of collected droplets $\phi_{0}$ and the normalization constant $r_{n}$. The Stokes' settling parameter $\kappa$ is a function of temperature (see appendix). In the following I extract all these parameters from the measurement and show that they have reasonable values.

I start with determining the decay rate $a$ of the number density $n(t)$ according to eq. (4.47). In fig. 4.11 the number density is shown for the measurement shown in fig. 4.9(a)-(c) together with an exponential fit in the decreasing part, where eq. (4.47) applies. This gives a decay rate of $a=9 \times 10^{-4} \mathrm{~S}^{-1}$, that amounts to a time of about $1000 \mathrm{~s}$ in which the droplets leave the measurement volume. This is a reasonable 


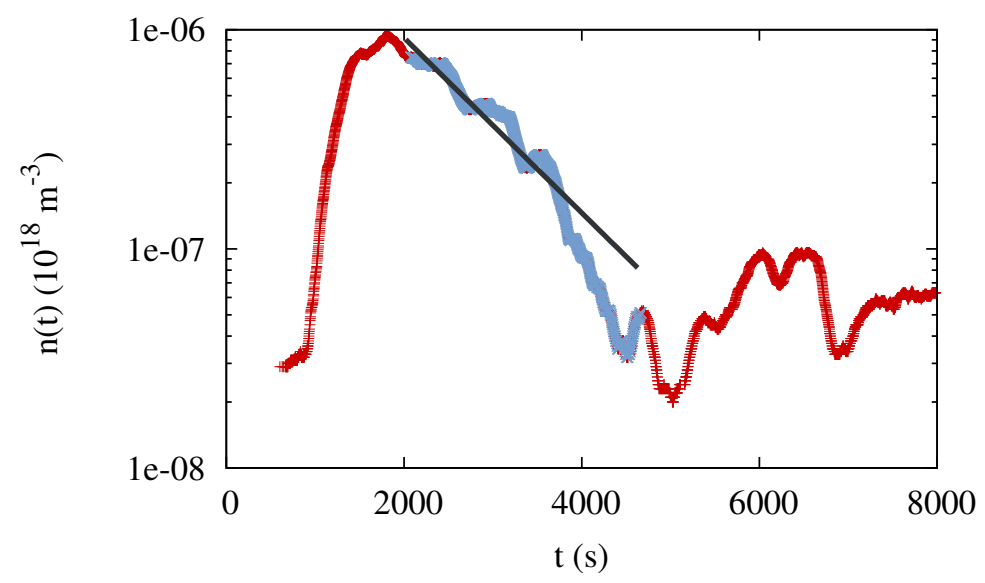

Figure 4.11: The decay of the total number density $n(t)$ (blue symbols) is fitted with an exponential function (black line) to determine the decay rate, $a=9 \times 10^{-4} \mathrm{~s}^{-1}$. The red crosses represent the data points of the whole measurement.

time scale, because for a typical sedimentation speed of around $10^{-5} \mathrm{~ms}^{-1}$ droplets can move in that time a distance of about $1 \mathrm{~cm}$, which is the height of the fluid layer. As the next parameter I determine the length scale $L$. The number density $n(r, t)$, eq. (4.46), is described by a function with one maximum. That maximum corresponds the second maximum of the bimodal size distributions in fig. 4.9(c). At the maximum a relation between the position of the peak $r_{p}$, the decay rate $a$ and the length scale $L$ emerges, because at $r_{p}$ the derivative of the exponent in eq. (4.46) with respect to $r$ should vanish

$$
\begin{aligned}
0 & =\frac{\mathrm{d}}{\mathrm{d} r}\left(\frac{4 r}{\phi_{0} L}+\frac{4 a}{\phi_{0} \kappa r}\right)_{r=r_{p}} \\
\Rightarrow L & =\frac{\kappa r_{p}^{2}}{a} .
\end{aligned}
$$

With $r_{p} \approx 20 \mu \mathrm{m}$ and $\kappa\left(27^{\circ} \mathrm{C}\right)=4 \times 10^{4} \mathrm{~m}^{-1} \mathrm{~s}^{-1}$ (see appendix) and $a=9 \times 10^{-4} \mathrm{~s}^{-1}$ the length scale $L$ is found to be $L=\kappa r_{p}^{2} / a \approx 1.8 \mathrm{~cm}$. As expected $L$ takes a value of the order of the sample height, i.e. $1 \mathrm{~cm}$ for the present experiment.

Finally I determine the volume fraction of collected droplets $\phi_{0}$. For large radii the second term in the exponent of eq. (4.46) can be neglected and the number density $n(r, t)$ decreases exponentially with respect to the radius. Thus fitting the decay length allows the determination of the parameter pair $\phi_{0} \cdot L$. The rescaled data in 
fig. 4.10 is fitted for $r>r_{p}$ with a decaying exponential function ${ }^{2}$. With $\phi_{0} \cdot L=7.7 \mu \mathrm{m}$ we estimate the volume fraction of collected droplets to be $\phi_{0}=7.7 \mu \mathrm{m} / L \approx 4 \times 10^{-4}$. To estimate if $\phi_{0}=4 \times 10^{-4}$ is a reasonable value I compare it to the volume fraction of Brownian particles in order to calculate the collection efficiency $\varepsilon$, for collisions of large droplets with smaller ones. The overall volume fraction in the present experiment is about $\phi_{\text {tot }} \approx 1 \times 10^{-2} \cdots 3 \times 10^{-2}$. From the two peaks in the size distribution (fig. 4.10) at about $10 \mu \mathrm{m}$ and $20 \mu \mathrm{m}$ we conclude that there is a factor of $2^{3}=8$ between the volume of Brownian particles and the volume of droplets that sediment and collect the Brownian particles. This gives a collection efficiency of $\varepsilon=\phi_{0} /\left(\phi_{t o t} / 8\right) \approx 0.3$. This value lies right in the range, $0.1 \leq \varepsilon \leq 1$, of collection efficiencies reported for other systems (Beard and Ochs, 1993).

\subsection{Discussion and summary}

Here I discuss the findings and insights of the present chapter. I start with the theory for large $k$ and conclude with the experimental observation of the size distribution.

\subsubsection{Theory}

In section section 4.2.2 we found three fixed points in the dynamics of the reduced aggregate radius $\rho=R /\langle R\rangle$. Figure 4.12, where the two fixed points for $\rho>0$ are shown, summarizes nicely the different parts of the dynamics with respect to $k$. A distribution as a function of the reduced radius lies on a vertical line at the respective $k$ value. The arrows indicate the time derivative of the reduced radius and show how the distributions will evolve.

For $k=1$ the dynamics is governed by an unstable fixed point at $\rho=1$. This is the case of Ostwald ripening, where the number of aggregates decays like $t^{-1}$ and a universal distribution can be found, that has a time independent shape as a function of the reduced radius, see section 1.3.

For $k=3 / 2$ the two fixed points exchange their stability and for $k>3 / 2$ the dynamics is governed by the stable fixed point at $\rho=1$. This leads to size focussing, because larger aggregates grow slower that smaller ones. All aggregates with a radius smaller than $\langle R\rangle \rho_{+}$, i.e. all aggregates with sizes that lie below the unstable fixed point, $\rho_{+}$, will evaporate.

For Ostwald ripening the decease in the number of aggregate does not affect the value of $k$, because $\xi=0$ (see eq. (4.6)). This changes, if $\xi>0$. Here a decrease of the aggregate number $N$ will lead to an increase of $k$. Thus whenever aggregates

\footnotetext{
${ }^{2}$ In principle the second term of the exponent in eq. (4.46), that is dominant for small radii has to be taken into account, because the fit starts right behind the maximum, where the two terms are equal. However, for an estimation of the parameter and in view of the scatter in the experimental data the present approximation is well justified.
} 


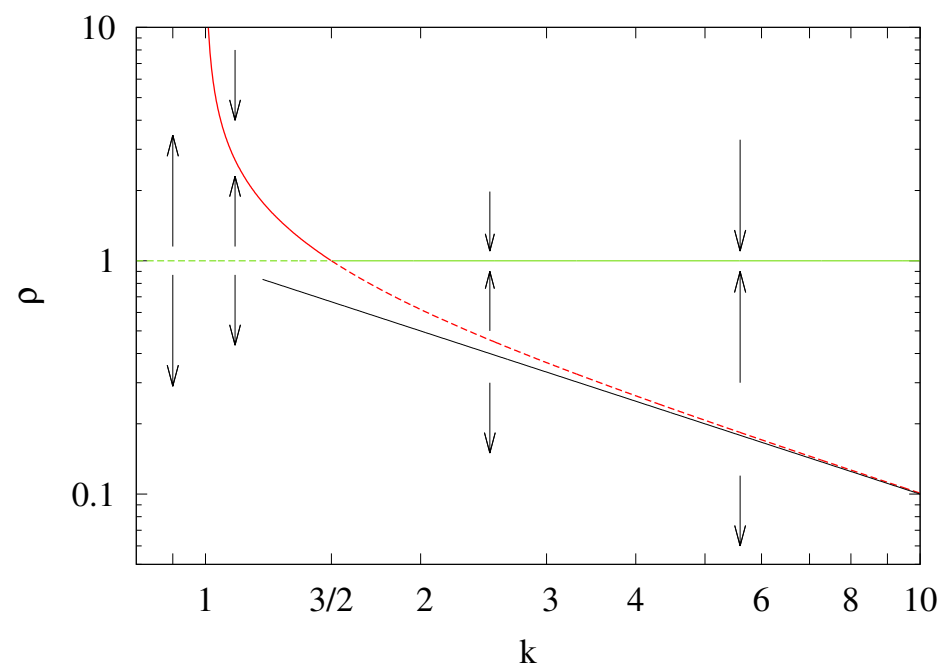

Figure 4.12: Phase portrait of the evolution of the reduced aggregate radius $\rho=R /\langle R\rangle$. The green line denotes a fixed point at $\rho=1$, and the red line the position of another fixed point, $\rho_{+}$. A thin straight black line has been added to show that $\rho_{+}$rapidly approaches $k^{-1}$ for $k \gtrsim 5$.

evaporate the $k$ value grows, until evaporation ceases and $k$ saturates at a constant value. Hence, the dynamics of Ostwald ripening is unstable to material flux.

Whenever the distribution evolves at a constant number of aggregates the shape of the distribution is time invariant, when the distribution is given as a function of $R^{2}-\left\langle R^{2}\right\rangle$ or for smaller $k$ values with a higher order term according to eq. (4.29). Hence, no universal distribution is approached, but the shape of the initial distribution is preserved in that coordinates, except for changes at small radii due to evaporation (see fig. 4.3).

To understand the appearance of size focussing for $k>1$ it is instructive to look at the radius dependent growth rates for different values of $k$. In fig. 4.13 the dimensionless growth rates are shown as a function of the reduced radius. Positive values indicate a growing aggregate and negative values a shrinking one. From analysis of eq. (4.6) one finds that the growth rate is zero for $\rho=1 / k$ and has a maximum at $\rho=2 / k$. For a given distribution it is now easy to find a $k$ value, such that no aggregates will evaporate, i.e. where even the smallest aggregate lies beyond the maximum of the growth rate: $\rho_{\min }>2 / k$. For this case the growth rate is a monotonically decreasing function of the radius. This implies that small aggregates grow faster that larger ones. Since the order of aggregate size is preserved, the distribution has to become narrower: the width of the distribution must decrease. 


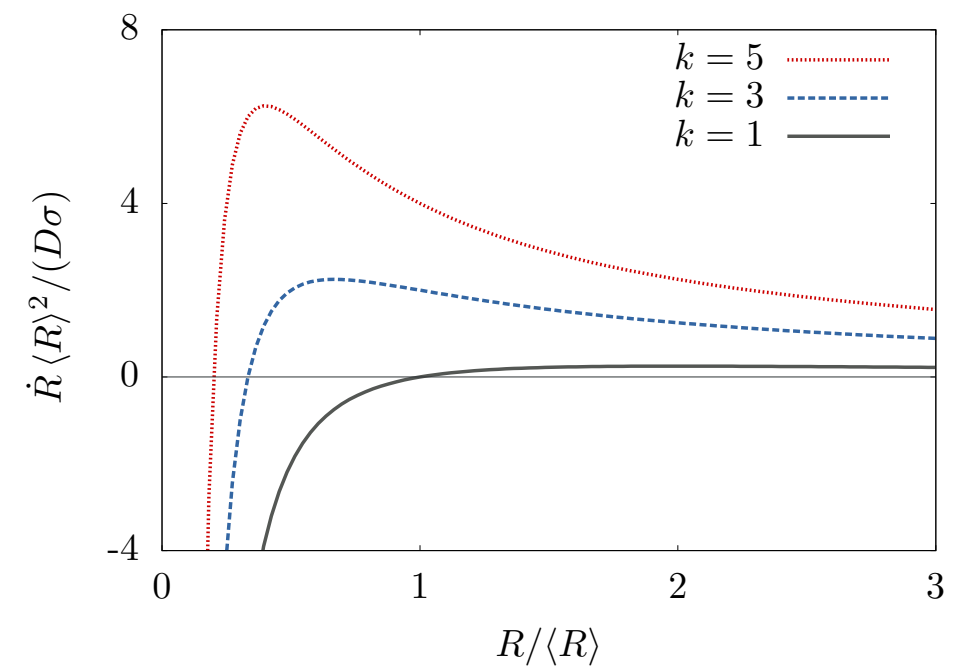

Figure 4.13: Dimensionless growth rate as a function of the reduced aggregate radius (according to eq. (4.6)) for different values of $k$ as indicated in the legend.

\subsubsection{Experiments}

Experimental observations of the size distribution of growing droplets in driven binary phase separation reveal that they grow as a bell-shaped distribution when they behave like Brownian particles. We observe a crossover to growth by collection. Droplets, whose motion is already affected by buoyancy, collect smaller ones. In the size distribution a second peak of the sedimenting droplets emerges, leading to a bimodal distribution. Eventually the distribution decays and becomes mono-modal again, leaving behind only a small amount of droplets, that behave like Brownian particles. This behaviour can also be observed in experiments, where the jump to zero driving happens at later times and the sedimentation of droplets goes along with an increase of droplet volume fraction, due to the driving.

As a summary the presence of gravity leads to an enhanced growth of large droplets, therefore introducing a mechanism of removing droplets from the assembly, see section 1.5. On this level we understand the evolution of the size distribution for one precipitation cycle. A sustained continuous driving will lead to repeated cycles and poses the question how to understand the time scales of droplet growth based on the findings of this chapter. 


\section{Oscillation periods for constant ramp rates}

The previous chapters provided the basis to investigate the parameter dependence of the oscillations occurring in continuously driven binary phase separation. I start with reporting on measurements of the oscillation period $\Delta t$ for constant ramp rates $\xi$. Subsequently, I investigate the influence of external flow. Based on these findings and the results of chapter 4, I develop and expand a model for the oscillation period (Lapp, 2011), and equip it with a more fundamental basis. The chapter culminates in a parameter-free data collapse for all data onto the master curve predicted by the model.

\subsection{Experimental results for the oscillation period}

\subsubsection{Measuring the oscillation period}

Fig. 5.1 compiles data of $\Delta t$ for a vast range of heating rates, $10^{-6} \mathrm{~s}^{-1}<\xi<10^{-3} \mathrm{~s}^{-1}$, and four different scenarios of phase separation in a binary mixture: a) the emergence and sedimentation of water-rich droplets in an isobutoxyethanol-rich phase; b) the emergence and rising of isobutoxyethanol-rich droplets in a water-rich phase; c) the emergence and sedimentation of methanol-rich droplets in a hexane-rich phase; and d) the emergence and rising of hexane-rich droplets in a methanol-rich phase.

Different data points for a given ramp rate are due to the drift of $\Delta t$ when pertinent material constants change upon moving further away from the critical point. In the appendix I provide the temperature dependence of the material constants, which in turn translates to a time dependence when inverting the protocol $T(t)$ of the temperature ramp. For all data the height of the layer was $h \approx 1 \mathrm{~cm}$. Measurements for samples with varying heights between $h=0.25 \mathrm{~cm}$ and $5.5 \mathrm{~cm}$ for the lower layer of IBE $+\mathrm{W}$ mixture showed that $\Delta t$ is hardly affected by $h$ (Lapp, 2011). The data points for the IBE $+\mathrm{W}$ mixture ${ }^{1}$ (left) are obtained by particle tracking (as described in section 2.5), and those for $\mathrm{M}+\mathrm{H}$ (right) refer to subsequent minima of turbidity

\footnotetext{
${ }^{1}$ The data points were obtained in collaboration with Tobias Lapp and also used in his Ph.D thesis (Lapp, 2011). However, the representation of the data in fig. 5.1 is based on a new parametrization of the ramp rates $\xi$ for which I now use the scaling representation of the phase diagram (see section 2.2).
} 

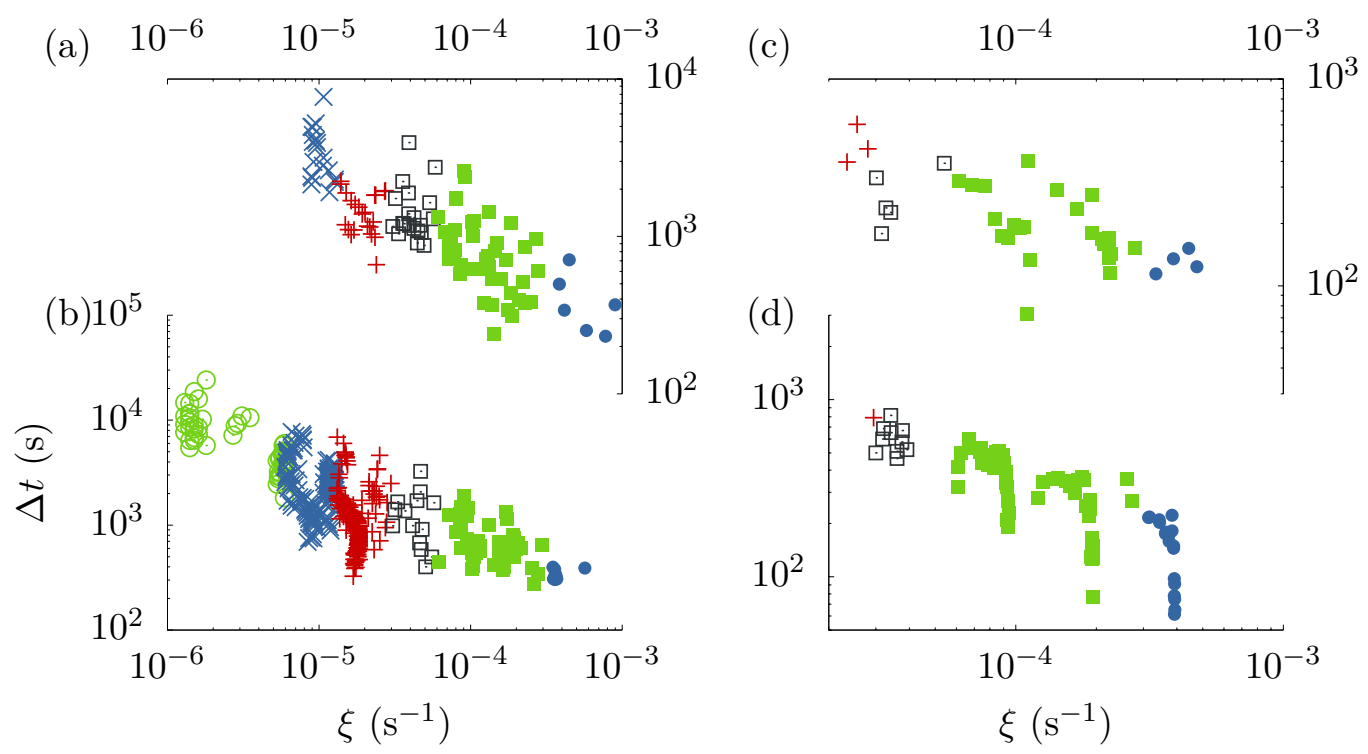

Figure 5.1: The oscillation period, $\Delta t$, plotted vs. the ramp rate $\xi$. The four panels show $\Delta t$ for (a) water-rich droplets in an IBE-rich continuous phase, (b) IBE-rich droplets in a water-rich continuous phase, (c) methanol-rich droplets in a hexane-rich continuous phase, and (d) hexane-rich droplets in a methanol-rich continuous phase, respectively. The colours and symbols encode different ranges of ramp rates $\xi$ : open green circle, $\xi<6 \times 10^{-6} \mathrm{~s}^{-1}$; blue cross, $10^{-6} \mathrm{~s}^{-1}<\xi \leq 1.3 \times 10^{-5} \mathrm{~s}^{-1}$; red plus, $1.3 \times 10^{-5} \mathrm{~s}^{-1}<\xi \leq 3 \times 10^{-5} \mathrm{~s}^{-1}$; open black square, $3 \times 10^{-5} \mathrm{~s}^{-1}<\xi \leq 6 \times 10^{-5} \mathrm{~s}^{-1}$; green square, $6 \times 10^{-5} \mathrm{~s}^{-1}<\xi \leq 3 \times 10^{-4} \mathrm{~s}^{-1}$; and blue circle, $3 \times 10^{-4} \mathrm{~s}^{-1}<\xi$.

in measurements described in section 2.4. I verified that both methods provide the same results. However, the data obtained from droplet tracking tend to be more accurate.

\subsubsection{Influence of flow}

Movies of the experiments (see supplementary online information of Rohloff et al. (2015)) clearly show that there is considerable convection in the background flow when the droplets sediment. The influence of this background flow in the bulk fluid has first been addressed by Vollmer et al. (2007). They used a three-storied hourglass-like cuvette as a sample cell (see inset of fig. 5.2) and measured the oscillation period in each of the three compartments. The motion of the bulk fluid depends on the shape and size of the cell. However, in spite of the considerable changes of the flow, they found no influence on the oscillation period (see fig. 5.2).

Here I will report on measurements conducted in collaboration with Julian Vogel, 


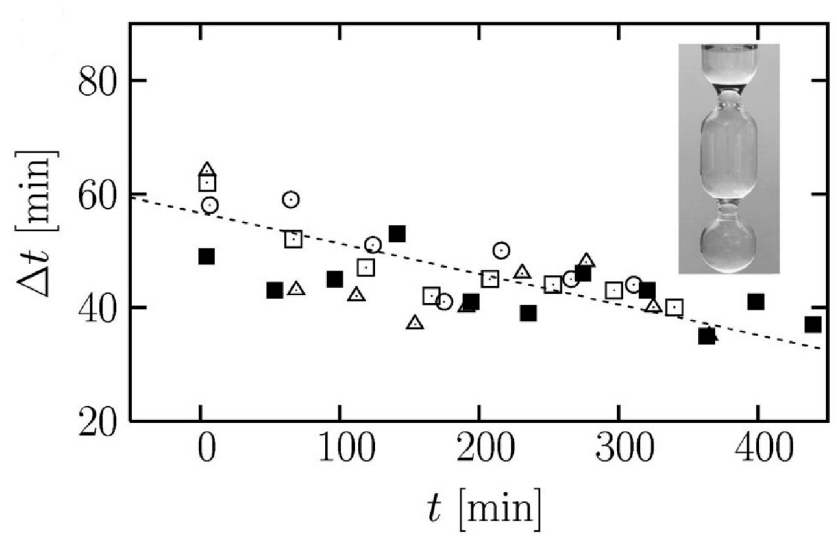

Figure 5.2: Measurements of the oscillation period of a polystyrene cyclohexane mixture. The different open symbols correspond to the oscillations in the lower $(\odot)$, middle $(\square)$ and upper $(\triangle)$ part displayed hour-glass shaped of the container $(4 \mathrm{~cm}$ high). The boxes $\mathbf{\square}$ show control measurements in a rectangular cuvette. Reproduced from Vollmer et al. (2007).

where the flow is systematically influenced by stirring with a magnetic stirrer at the bottom of the lower layer. To not destroy the oscillations completely we had to use a small stirring bar: an almost spherical one of $2 \mathrm{~mm}$ diameter and $2 \mathrm{~mm}$ length. As described in section 2.4 the oscillation period is extracted from turbidity measurements of IBE $+\mathrm{W}$ mixtures. Figure 5.3 shows the oscillation periods for stirring rates ${ }^{2}$ of $\omega=1$ to $3 \mathrm{~Hz}$ together with with the reference data of undisturbed oscillations, $\omega=0 \mathrm{~Hz}$. Within experimental scatter the oscillation periods are not affected by the stirring.

I conclude that the oscillation period is not influenced by the flow in accordance with earlier experiments of Vollmer et al. (2007). In addition to this qualitative finding, I add here the following observations from our experiments, where the strength of the flow has been varied independently from other parameters by tuning the stirring rate. In fig. 5.4 three space-time plots are shown for increasing stirring rate. For the measurement of $1 \mathrm{~Hz}$ the oscillations are not visible in the first part of the measurement: up to $120 \mathrm{~min}$, there only is a slight turbidity while oscillations emerge at later times. For the stirring rate of $5 \mathrm{~Hz}$ the lower layer is turbid throughout the whole measurement, all oscillations are gone. However, the flow induced by the stirring is not yet strong enough to influence the meniscus. Consequently, we still observe oscillations in the upper layer. In the lower layer the constant driving produces droplets, which are present throughout the whole measurement. Moreover, the droplet volume is still transferred to the upper phase. Hence, the position

\footnotetext{
${ }^{2}$ Our present stirring device does not allow stirring rates smaller than 50 revolutions per minute, i.e. $0.833 \mathrm{~Hz}$.
} 
(a)

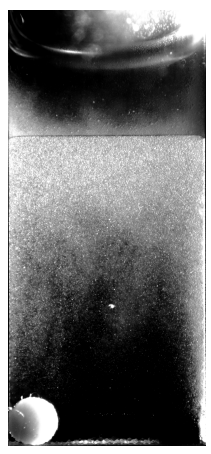

(b)

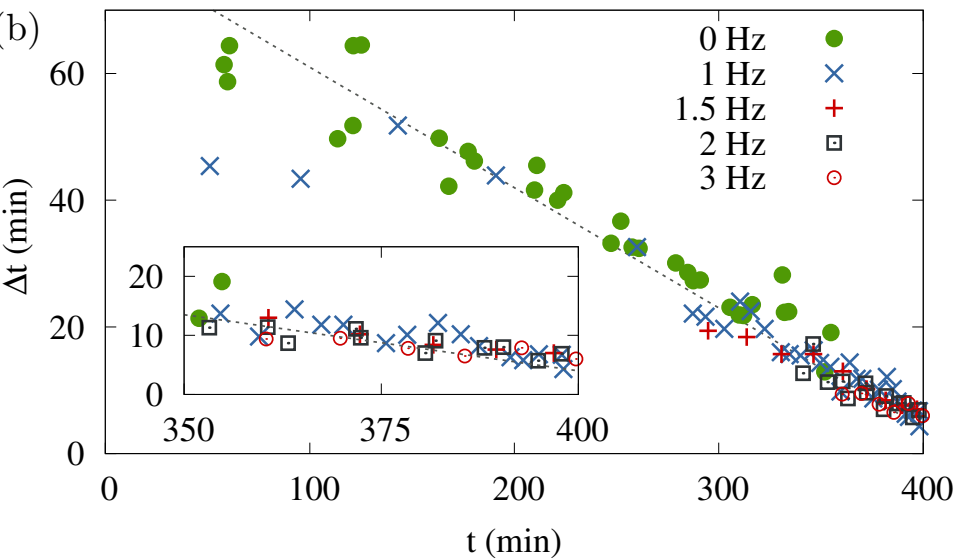

Figure 5.3: (a) Snapshot of sample during the experiment. At the bottom the $2 \mathrm{~mm}$ large stirring bar is visible. (b) Oscillation periods in the lower layer of an IBE $+\mathrm{W}$ mixtures for different stirring rates. For all data a ramp rate $\xi_{\text {poly }}=2.5 \times 10^{-5} \mathrm{~s}^{-1}$ was used ${ }^{3}$. The dotted line serves as guide to eye, and the inset show the same data points for the last $50 \mathrm{~min}$ of the measurement.

of the meniscus decreases in a response to the growth in height of the upper layer. The flux of droplet volume from the lower to the upper layer happens here in a steady fashion. This suggests that the stirring interferes with the mechanism, which periodically clears up the sample and removes droplets from the lower layer, i.e. the explosive droplet growth by collection of small droplets.

To estimate the magnitude of frequency of the stirring bar, at which the induced flow disturbs the growth by collection, I compare two time scales. The first one is the time scale of the large scale convection induced by the stirring: $t_{c}=l_{c} / v_{c}$, where $l_{c} \approx 10^{-2} \mathrm{~m}$ is the height of the fluid layer and $v_{c} \approx \omega \cdot 10^{-3} \mathrm{~m}$ the velocity at the surface of the stirring bar with a perimeter of about $10^{-3} \mathrm{~m}$. For the second time scale I consider the time in which a large droplet collects the small one: $t_{s}=\Lambda / v_{s}$. It is calculated based on the settling velocity $v_{s}$ and a typical droplet distance $\Lambda$. For a droplet with a radius of $20 \mu \mathrm{m}$ and a settling parameter $\kappa=4 \times 10^{4} \mathrm{~m}^{-1} \mathrm{~s}^{-1}$ (see appendix) eq. (4.33) gives a settling velocity of $v_{s} \approx 10^{-5} \mathrm{~ms}^{-1}$. A typical droplet distance is inferred from the number density $n: \Lambda=n^{-1 / 3}$. For $n \approx 10^{12} \mathrm{~m}^{-3}$ (see e.g. fig. 4.11) we obtain $\Lambda=10^{-4} \mathrm{~m}$. If the flow has the same direction as the sedimentation speed, the distance of the two droplets decreases and they meet in

\footnotetext{
${ }^{3}$ The experiments in fig. 5.3 and fig. 5.4 were done with temperature ramps base on the polynomial and the scaling representation of the phase diagram, respectively. The experiments have approximately the same duration. This is achieved by choosing $\xi_{\text {scaling }} / \xi_{\text {poly }}=2 / 2.5=0.8$, see fig. 2.4 where the value of 0.8 corresponds roughly to the averaged driving.
} 
(a)

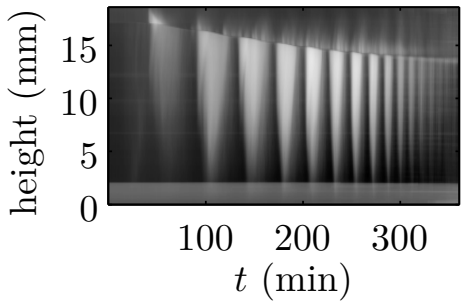

(b)

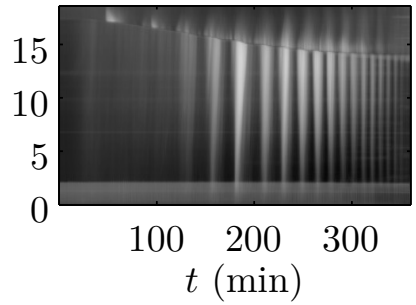

(c)

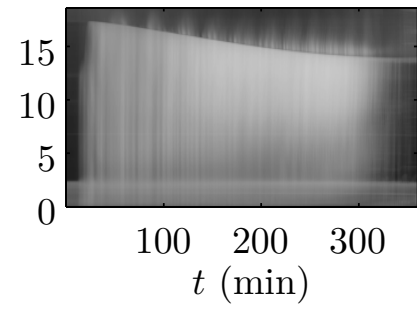

Figure 5.4: Space-time plots for different stirring rates measured in IBE $+\mathrm{W}$ mixtures for $\xi_{\text {scaling }}=2 \times 10^{-5} \mathrm{~s}^{-1}$ : (a) $0 \mathrm{~Hz}$, (b) $1 \mathrm{~Hz}$, and (c) $5 \mathrm{~Hz}$. The bright vertical stripe at the bottom is due to the stirring bar (cf. fig. 5.3(a)), that changes the average grey level at the bottom $2 \mathrm{~mm}$ of the pictures.

the time $t_{s}$. If the direction of the flow is reversed, then the distance will increase. The reversal of the bulk flow happens on the time scale of the convection $t_{c}$. Thus, for $t_{s}>t_{c}$ the collection mechanism is disturbed by the external flow. Putting all numbers together, leads to

$$
\omega>\frac{l_{c} v_{s}}{\Lambda 10^{-3} \mathrm{~m}} \approx 1 \mathrm{~Hz} .
$$

This is the frequency at which the flow is expected to suppress the oscillations. Remarkably, the oscillations are indeed suppressed for $\omega=1 \mathrm{~Hz}$, but only for the first 100 min (see fig. 5.4(b)). This is due to the temperature dependence of the settling velocity. It increases when the temperature is moved away from the critical point, and the mass density contrast of the droplets increases. Therefore, it is still possible to observe oscillations for frequencies of $\omega=3 \mathrm{~Hz}$ for times $t>300 \mathrm{~min}$, where the temperature has increased sufficiently (see fig. 5.3). To get a more accurate relation between the stirring frequency and the time until the oscillations are suppressed, particle image velocimetry (PIV) or particle tracking can be applied to characterise the external flow. This investigation should ideally be done in a setup where one superimposes the inherent background flow with a still better defined flow pattern as it is yet possible with the stirring bar.

In summary, the flow destroys the oscillations, if it becomes too large. However, whenever oscillations are present, their period is not influenced by the flow. This has immediate consequences on the modelling for the oscillation period, which will be developed in the following section.

\subsection{Calculating the oscillation period for constant $k$ values}

In this section a model for the oscillation period is developed. It will be based on the growth laws for the largest droplets of the size distribution. 


\subsubsection{Evolution of the radius of the largest droplets}

I start with general considerations motivating the setup of the model.

1. Spatial degrees of freedom need not be considered to describe the evolution of the largest droplets. In the previous section 5.1.2 it was shown that neither the naturally present convection nor an external flow (when not destroying the oscillation), has an impact on the time scale of the oscillation period. Furthermore, for the nonlinear reactions terms characterising phase separation the convective mixing efficiently eliminates spatial inhomogeneities of the droplet size distribution (Benczik and Vollmer, 2010, 2012). Indeed, based on visual inspection of the accompanying movies, we estimate the mixing time scale to be of the order of seconds. It is about three orders of magnitude smaller than the period $\Delta t$.

2. It is sufficient to consider the characteristic size of the largest droplets rather than the full droplet size distribution. For diffusively growing droplets the size distribution is sharply bounded towards large droplets. Consequently, the largest droplets in the system have a well-defined size and there are only few of these droplets (Slezov, 2009; Clark et al., 2011; Vollmer et al., 2014). When buoyancy starts to effect their motion these large droplets collect smaller droplets, grow rapidly, and eventually clear the system from droplets by precipitation (Kostinski and Shaw, 2005).

3. I will show in the following that a quantitative description of $\Delta t$ can be achieved when only considering droplet growth by diffusive accretion, that relaxes supersaturation provided by the constant driving, $\xi$, and the collection of small droplets by sedimenting large ones. The processes are illustrated in fig. 5.5, and I will now discuss them in turn. For later reference I will also revisit the predictions of a scenario where small droplets grow predominantly by Ostwald ripening (Wilkinson, 2014).

\subsubsection{Growth by accretion of material provided by the constant driving, $\xi$}

The dynamics of large droplets crossing the meniscus (Aarts et al., 2005) and droplet nucleation (Binder and Stauffer, 1976; Farjoun and Neu, 2011) provide microscopic droplets in the fluid. Subsequently, the supersaturation in the bulk relaxes by diffusion of the minority component onto the droplets. The diffusive accretion of material on the droplets relaxes the supersaturation provided by the driving, $\xi$, which thus induces droplet growth.

In the experiments the temperature ramp is adjusted in such a way that the volume fraction of droplets grows linearly in time with a speed $\xi$ (cf. section 2.3). In chapter 4 


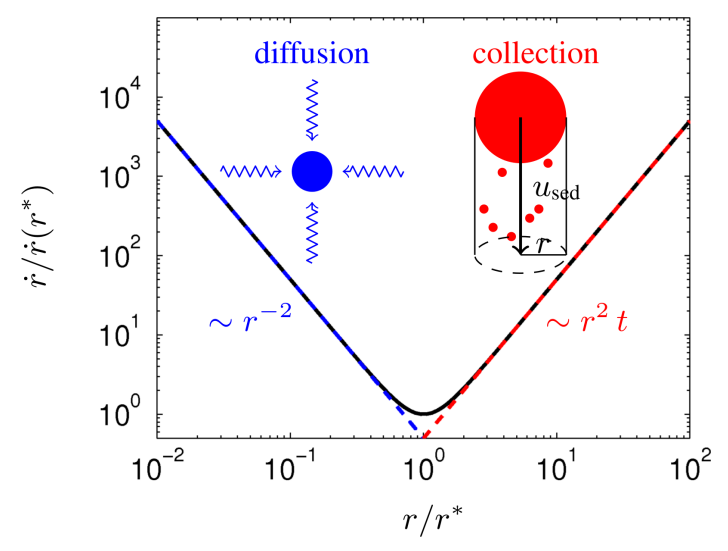

Figure 5.5: Dominant growth mechanisms (insets) and the bottleneck in droplet growth (main panel). As a function of droplet size $r$ the growth speed $\dot{r}$ of droplets shows a sharp minimum at a size $r^{*}$. Smaller droplets grow by diffusion. The growth of larger droplets is accelerated by sedimentation that promotes the collection of small droplets.

the growth of droplet assemblies under these conditions was investigated. It was shown for large values of $k$, which are observed in our experiments (cf. section 4.3.1), that the number density of droplets is preserved. Droplets of a characteristic radius $r$ and number density $n$ occupy a volume fraction $n 4 \pi r^{3} / 3$. When the droplet volume fraction increases with speed $\xi$ and the number density $n$ is conserved, diffusive growth provides a temporal change of the droplet radius

$$
\frac{\mathrm{d}}{\mathrm{d} t} \frac{4 \pi n r^{3}}{3}=\xi \quad \Rightarrow \quad \dot{r}=\frac{\xi}{4 \pi n} \frac{1}{r^{2}} .
$$

Alternatively, this growth law can be obtained as large $k$ approximation of the diffusive growth law (see eq. (1.10))

$$
\dot{R}=\frac{\sigma D}{R^{2}}\left(k \frac{R}{\langle R\rangle}-1\right), \quad k=1+\frac{\xi}{4 \pi \sigma D n} .
$$

It was shown in section 4.3.1 that $k$ takes values of the order to $10^{6}$ under the conditions considered here, and that $R \simeq\langle R\rangle$ in the late stages of competitive droplet growth at large $k$. Hence, eq. (5.3) reduces to eq. (5.2).

\subsubsection{Growth by Ostwald ripening}

Droplet growth by classical Ostwald ripening is described by eq. (5.3) for $k=1$ and the solutions approach an asymptotic scaling solution where $0 \leq R /\langle R\rangle \leq 3 / 2$ (Lifshitz and Slyozov, 1961). Evaluating eq. (5.3) for $k=1$ and large droplets with 
radius $r$ yields

$$
\dot{r}=\delta \frac{D \sigma}{r^{2}},
$$

where $\delta$ accounts for the $(R /\langle R\rangle-1)$ term in (5.3). Lifshitz and Slyozov (1961) predicted that $R /\langle R\rangle \leq 3 / 2$ such that $\delta \simeq 1 / 2$ for the largest droplets in the system. Wilkinson (2014) uses $\delta=1$, in accordance with observations at finite droplet volume fractions (Marder, 1987). For very large volume fractions values up to $\delta \simeq 1.5$ have been observed experimentally (Snyder et al., 2001), and $\delta \simeq 2$ can be reached when the diffusive collection of supersaturation becomes anisotropic due to flow effects (Ratke and Host, 1991).

Upon introducing the definition (5.3) of $k$ into eq. (5.2) it becomes apparent that the diffusive growth of the largest droplets in the system follows the same $\dot{r} \sim D \sigma r^{-2}$ scaling irrespective of whether supersaturation is provided by the constant driving, $\xi$

$$
\dot{r}=\frac{\xi}{4 \pi n} \frac{1}{r^{2}}=(k-1) \frac{D \sigma}{r^{2}} \quad \text { (constant driving) }
$$

or by evaporation of small droplets in Ostwald ripening,

$$
\dot{r}=\delta \frac{D \sigma}{r^{2}} \quad \text { (Ostwald) }
$$

The scenarios differ qualitatively in the evolution of the droplet number which remains constant in the former case, such that also $k$ takes a constant value, while it decays like one over time for Ostwald ripening. However, as the growth of the largest droplets is concerned, the growth laws, eqs. (5.5a) and (5.5b), only differ by the numerical value of the constant factors $k-1$ and $\delta$, respectively. In section 4.3 .1 it was shown that $k$ takes values of the order of $10^{6}$ for the experiments discussed here. Hence Ostwald ripening is expected not to be a relevant process. Remarkably, however, a theoretical treatment of the parameter dependence of $\Delta t$ based on eq. (5.4), as provided by Wilkinson (2014), will provide the same results as the present analysis, except for quantitative differences arising from the vastly different numerical values taken by $\delta$ and $k$. In the following I will only deal with the case of constant driving. The analogous results for an experimental system where Ostwald ripening appears to be important can be obtained by substituting $(k-1)$ by $\delta$.

\subsubsection{Growth by collection of smaller droplets}

When the droplets become sufficiently large, they drift under the influence of buoyancy forces as described in section 4.3.2. Large droplets collide with smaller ones and collect their volume. The according growth law is derived in eq. (4.34). In the present setting the overall volume fraction of droplets grows linearly in time $\phi=\xi t$. We introduce the collection efficiency $\varepsilon$, that describes how much volume is effectively 
collected by the large droplets. Therefore we get from eq. (4.34) the collisional growth rate

$$
\dot{r}=\frac{\varepsilon \kappa \xi t}{4} r^{2} .
$$

Observe that $r$ refers to the radius of the largest droplets in the system - a minute minority of droplets that accounts for only a small part of the droplet volume fraction.

\subsubsection{The bottleneck of droplet growth}

The diffusive growth mechanisms, eq. (5.5), work very well for small droplets due to the factor $r^{-2}$, and they becomes less and less efficient when $r$ grows. In contrast, growth by collecting small droplets, eq. (5.6), does not contribute to the growth as long as all droplets are small, while it leads to runaway growth of the large droplets when their motion is affected by buoyancy. Hence, I assert that the sum of the dominant diffusive-growth contribution, eq. (5.5a), and the contribution accounting for the collection of smaller droplets, eq. (5.6),

$$
\dot{r}=(k-1) \frac{\sigma D}{r^{2}}+\frac{\varepsilon \kappa \xi t}{4} r^{2}
$$

faithfully describes the growth of the largest droplets in the system. The growth law, eq. (5.7), shows a bottleneck of growth at the bottleneck radius, $r^{*}$, where the droplet growth speed, $\dot{r}$, takes its smallest value, $\dot{r}\left(r^{*}, t^{*}\right)=(2 / 3)\left(r^{*}\right)^{-2}$ (see fig. 5.5),

$$
r^{*}=r\left(t^{*}\right)=\left(\frac{4(k-1) \sigma D}{\varepsilon \kappa \xi t^{*}}\right)^{1 / 4} .
$$

The bottleneck is approached at the time $t^{*}$ required for droplets to grow from zero radius to the radius $r^{*}$. Integrating eq. (5.5a) from $r=0$ to $r=r^{*}$ yields $r^{* 3}=3(k-1) \sigma D t^{*}$. Together with eq. (5.8) this equation provides the following expressions for the bottleneck time $t^{*}$ and the bottleneck radius $r^{*}$,

$$
t^{*}=\left(\frac{2^{6}}{3^{4}} \frac{[(k-1) D \sigma]^{-1}}{(\varepsilon \kappa \xi)^{3}}\right)^{1 / 7}, \quad r^{*}=\left(\frac{12[(k-1) D \sigma]^{2}}{\varepsilon \kappa \xi}\right)^{1 / 7} .
$$

Henceforth, I measure time in units of $t^{*}$, droplet radii in units of $r^{*}$, and, for conciseness of the notation, I denote the resulting dimensionless units still as $(r, t)$. In terms of these dimensionless variables eq. (5.7) takes the form

$$
\dot{r}=\frac{1}{3 r^{2}}+\frac{t r^{2}}{3},
$$

such that the growth velocity $\dot{r}(r, t)$ takes its minimum at $(r, t)=(1,1)$. 


\subsubsection{Calculating the period $\Delta t$}

As long as buoyancy does not yet affect the motion of the largest droplets in the system, the droplets grow diffusively by collecting supersaturation. In leading order for small droplets one can then neglect the growth contribution $t r^{2} / 3$ in eq. (5.10). For an initial droplet size $r(t=0)=0$ this entails

$$
\dot{r} \simeq \frac{1}{3 r^{2}} \quad \Rightarrow \quad r_{S}(t) \simeq t^{1 / 3},
$$

where the index $S$ in $r_{S}(t)$ stresses that the approximation applies as long as droplets are small, $r_{S} \lesssim 1$. As shown by the dotted line in fig. 5.6(a) this approximation provides a good estimate for values $t<1 / 2$.

Similarly, for large droplets the contribution $\left(3 r^{2}\right)^{-1}$ to the growth is sub-dominant in eq. (5.10) such that in leading order

$$
\dot{r} \simeq \frac{r^{2} t}{3} \Rightarrow r_{L}(t) \simeq \frac{6}{\Delta t^{2}-t^{2}} .
$$

Here, the index $L$ in $r_{L}(t)$ indicates that this solution applies when the droplets are large, $r_{L} \gtrsim 1$. The growth law, eq. (5.11b), features a finite-time singularity when $t$ approaches $\Delta t$. At the latest at this late time, the large droplets will rapidly fall out of the measurement window, such that the system is reset to its initial state $r \simeq 0$. On the one hand, the dash-dotted line in fig. 5.6(a) shows that eq. (5.11b) provides a very good description of the numerical data for $t \gtrsim t^{*}$ for the choice $\Delta t=2.44$. On the other hand, the expression eq. (5.11b) can not be matched continuously to eq. (5.11a) because for $\Delta t=2.44$ the latter expression produces smaller values for $r(t)$ for all $t$. Rather, a continuous and differentiable interpolation from eq. (5.11a) to eq. (5.11b) requires to choose

$$
r(t) \simeq \begin{cases}t^{1 / 3} & \text { for } t \leq 1 \\ 6\left(7-t^{2}\right)^{-1} & \text { for } t \geq 1\end{cases}
$$

The resulting first order estimate for $r(t)$ is shown by the dashed blue line in fig. 5.6(a). It diverges at $\Delta t=\sqrt{7} \simeq 2.646$, thus overestimating the time $\Delta t$ required to reach the finite-time singularity observed in the numerical data by about $8 \%$. A more accurate description of the numerical solution of eq. (5.10) is obtained by taking into account the leading order corrections of eqs. (5.11a) and (5.11b). A refined estimate for the droplet growth is obtained by using $r_{S}(t)$ to approximate the sub-leading contribution to the growth of $r^{3}$ by $t r^{4} \simeq t^{7 / 3}$. The resulting solution of eq. (5.10) becomes

$$
\frac{\mathrm{d}}{\mathrm{d} t} r^{3} \simeq 1+t^{7 / 3} \Rightarrow r_{s}(t) \simeq\left(t+\frac{3}{10} t^{10 / 3}\right)^{1 / 3}
$$


(a)

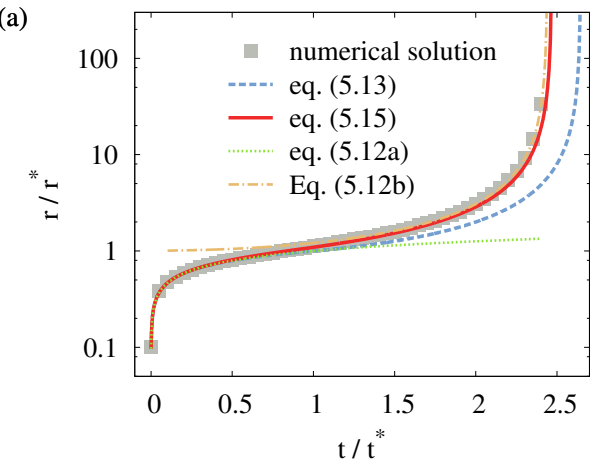

(b)

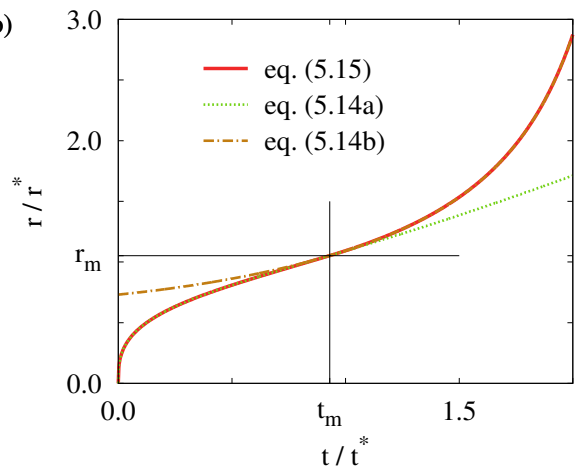

Figure 5.6: Finite-time divergence of droplet growth. (a) Comparison of the numerical solution of eq. (5.10) (grey squares) to the analytical approximations, eq. (5.12) (dashed blue line) and eq. (5.14) (solid red line), respectively. The green dotted line shows the leading order approximation for small droplets, eq. (5.11a), and the brown dash-dotted lines the description of the divergence of the size of large droplets, eq. (5.11b), evaluated for $\Delta t=2.44$. The analytical description of the droplet growth, (solid red line), is also shown in panel (b) which shows how eq. (5.14) is obtained by matching the expressions eqs. (5.13a) and (5.13b) for small and large droplets, respectively.

This expression provides an excellent fit to the numerical data for $t \lesssim t^{*}$, as shown by the the dotted green line in of fig. 5.6(b). For the large droplets a more accurate prediction is obtained by using $r_{L}(t)$ to approximate the $\left(3 r^{2}\right)^{-1}$ term in eq. (5.10),

$$
\begin{aligned}
-\frac{\mathrm{d}}{\mathrm{d} t} r^{-1} & =\frac{t}{3}+\frac{1}{3} r^{-4} \simeq \frac{t}{3}+\frac{1}{3}\left(\frac{\Delta t^{2}-t^{2}}{6}\right)^{4} \\
\Rightarrow r_{l}(t, \Delta t) & \simeq\left[\frac{\Delta t^{2}-t^{2}}{6}\right. \\
& \left.+\frac{(\Delta t-t)^{5}}{3 \cdot 6^{4}}\left(\frac{t^{4}}{9}+\frac{5 t^{3} \Delta t}{9}+\frac{23 t^{2} \Delta t^{2}}{21}+\frac{65 t \Delta t^{3}}{63}+\frac{128 \Delta t^{4}}{315}\right)\right]^{-1} .
\end{aligned}
$$

When evaluated at $\Delta t=2.467$ the expressions eqs. (5.13a) and (5.13b) match continuously and differentiable at the point $\left(t_{m}, r_{m}\right)=(0.9304,1.0526)$, [fig. 5.6(b)]

$$
r(t) \simeq \begin{cases}r_{s}(t) & \text { for } t \leq t_{m}=0.9304 \\ r_{l}(t, \Delta t=2.4667) & \text { for } t \geq t_{m}=0.9304 .\end{cases}
$$

The thick solid red lines in both panels of fig. 5.6 show the expression eq. (5.14) over the full $t$-range. It provides an excellent description of the numerical solution of eq. (5.10) that is shown by grey squares. In particular, the position of the predicted 
finite-time singularity, $\Delta t=2.467$, is only off by one percent from the numerically obtained value, $\Delta t=2.44$

In conclusion, the parameter dependence of the time scale, $\Delta t$, for the growth from vanishingly small to very large droplets is provided by the time, $t^{*}$, required to grow to the bottleneck size, $r^{*}$. Based on eq. (5.9) and the fit of $\Delta t$ in eq. (5.11b) to match the asymptotics of the numerical data shown in fig. 5.6, I find

$$
\begin{aligned}
\Delta t & \simeq 2.44 t^{*} \simeq 2.36\left(\frac{[k D \sigma]^{-1}}{(\varepsilon \kappa \xi)^{3}}\right)^{1 / 7} \\
& \simeq 3.39\left(\frac{n / \xi}{(\varepsilon \kappa \xi)^{3}}\right)^{1 / 7}
\end{aligned}
$$

where I inserted the definition 5.3 of $k$ to perform the last step. Note that $k \gg 1$ for our experiments (see section 4.3.1). A first hint that this prediction might be faithful is obtained by observing that the parts of a period where we observe high and low turbidity in fig. 2.6 are of comparable extent. This is consistent with the theoretical prediction that the singularity arises at $\Delta t=2.44 t^{*}$. In the following section this prediction will be compared to experimental data.

\subsection{Comparison to experimental data}

Here I compare the predicted dependence on the material parameters of the oscillation period, eqs. (5.15a) and (5.15b), to the experimental data shown in section 5.1. Our starting point is a data set where both the oscillation period and the number density, $n$, is known. This part is followed by data, from turbidity measurements, where the number density is not known. Finally the data of the stirring experiments are compared to the prediction.

\subsubsection{Master plots for droplet tracking data}

For the comparison of the prediction for the oscillation period $\Delta t$, eqs. (5.15a) and $(5.15 \mathrm{~b})$, with experimental data, I use here data where the time evolution of the droplet density was followed by particle tracking such that both, $\Delta t$ and $n$, are known from the experiment (see section 2.5). The data determining the temperature dependence of $\kappa, D$ and $\sigma$ is provided in the appendix. Hence, the collection efficiency, $\varepsilon$, remains as the only free parameter of the prediction, eqs. (5.15a) and (5.15b). In fig. 5.7(a) the relation between $\Delta t, n, \xi$ and $\kappa$ is plotted according to eq. (5.15b). The theoretical curves show the prediction eq. (5.15b) for the constant values, $\varepsilon=0.1$, 0.3 , and 1 , respectively. These values correspond to the middle and the respective most extreme values observed for other systems (Beard and Ochs, 1993), where the 
(a)
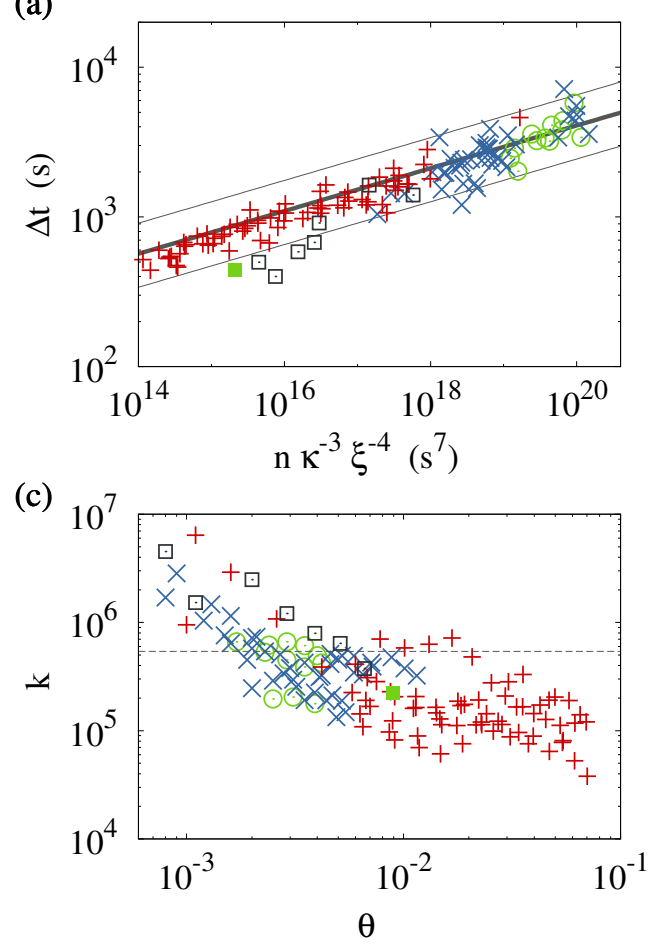

(b)

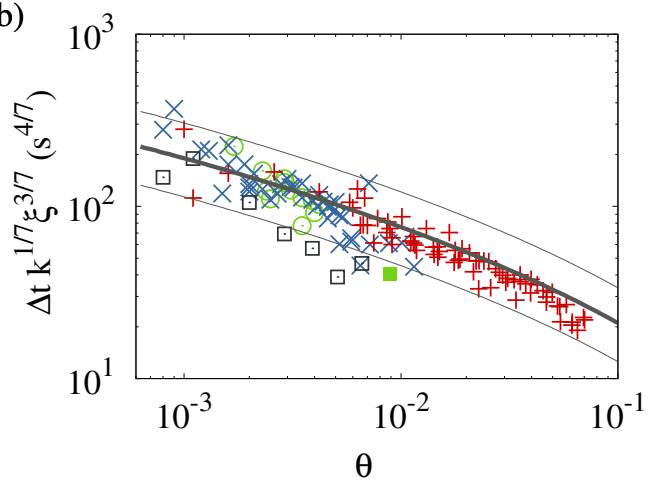

(d)

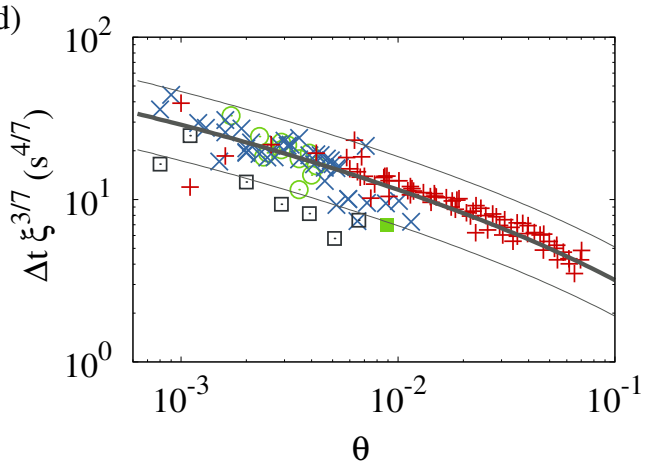

Figure 5.7: Master plots for the lower layer of IBE $+\mathrm{W}$, where period $\Delta t$ and the number density $n$ are known from particle tracking. All panels show the same data set for different combinations of parameters. The symbols encode different ramp rates $\xi$ as introduced in fig. 5.1. (a) The oscillation periods $\Delta t$ collapse when plotted as a function of $n \kappa^{-3} \xi^{-4}$. The solid lines represent the prediction, eq. (5.15b), where $\varepsilon=0.3$ is used for the thick line and the thinner lines above and below correspond to the choices $\varepsilon=0.1$ and $\varepsilon=1$, respectively. (b) The data also collapse as a function of the reduced temperature $\theta$ when $\Delta t k^{1 / 7} \xi^{3 / 7}$ is plotted. As before the solid lines correspond to the different choices of the collection efficiency $\varepsilon$. Panel (c) shows the dependence of $k$ on the reduced temperature $\theta$. The dashed line represents a typical value $k=5.4 \times 10^{5}$. (d) Replacing the temperature dependence of $k$ with a typical value $k=5.4 \times 10^{5}$ allows a data collapse and a prediction of the temperature dependence of $\Delta t$, even without referring to the number density $n$, eq. (5.16). 
collection efficiency $\varepsilon$ was reported to take values in the range $0.1 \leq \varepsilon \leq 1$. See also section 4.3.4 where the collection efficiency for our system is estimated to be $\varepsilon \approx 0.3$. All data points lie in the narrow band around the prediction, well within the uncertainty of $\varepsilon$. There only is a slight systematic mismatch of the slope. We attribute this trend to a weak temperature dependence of $\varepsilon$. The mismatch arises from a correlation of $\kappa$ and $\varepsilon$.

The coefficients $D, \sigma$ and $\kappa$ in eq. (5.15a) are functions of material constants. They show a strong temperature dependence that arises from the disappearance of the interfacial tension and the mass density contrast at the critical temperature, $T_{c}$, of the phase transition. This, in turn, entails the disappearance of $\sigma$ and $\kappa$ which are proportional to the interfacial tension and the mass density contrast, respectively (see appendix). Hence, eq. (5.15a) suggests that $\Delta t k^{1 / 7} \xi^{3 / 7}$ should be a function of the reduced temperature $\theta=\left|T-T_{c}\right| / T_{c}$. The proposed data collapse as a function of the reduced temperature is shown in fig. 5.7(b). Again the uncertainty in $\varepsilon$ induces a vertical displacement of the prediction on the logarithmic scale. The values for $k$ are shown in fig. 5.7(c), they are distributed over two orders of magnitude and decrease for increasing reduced temperature $\theta$.

\subsubsection{Master plots for turbidity data}

However, the period $\Delta t$ depends on $k$ only with the power of $1 / 7$ (see eq. (5.15a)). This weak dependence will change the prediction of the period by a factor of about $100^{1 / 7} \approx 2$. When suppressing the temperature dependence of $k$ in eq. (5.15a), i.e. when replacing $k$ by a typical value see fig. $5.7(d)$. From eq. (5.15a) we get

$$
\Delta t=\alpha\left(D \sigma \kappa^{3}\right)^{-1 / 7} \xi^{-3 / 7} \quad \text { with } \quad \alpha=2.36 k^{-1 / 7} \varepsilon^{-3 / 7} .
$$

Choosing $\varepsilon=0.3$ and a typical value of $k=5.4 \times 10^{5}$ results in $\alpha=0.6$, which is used for the prediction in fig. 5.7(d). The parameter $\alpha$ takes typical values for each type of mixture and has to be determined by fitting, if the number density is not available. In fig. 5.8 this has been done for the data points compiled in fig. 5.1. We find a remarkable collapse, when $\Delta t \xi^{3 / 7}$ is plotted as a function of the reduced temperature, and the resulting temperature dependence is faithfully described by the master curves, eq. (5.16), for $\alpha=0.6(\mathrm{IBE}+\mathrm{W})$ and $\alpha=0.9(\mathrm{M}+\mathrm{H})$, respectively.

\subsubsection{Influence of flow}

As a last part of the comparison of the experimental data with the prediction I plot the data set of the stirring measurements together with the master plot of the lower layer of IBE $+\mathrm{W}$ (see fig. 5.8(b)). Also the data for the measurements of the oscillation period with different stirring rates (fig. 5.3) collapse in a presentation like fig. 5.8 and lie right on top of the data points for unstirred measurements, see 

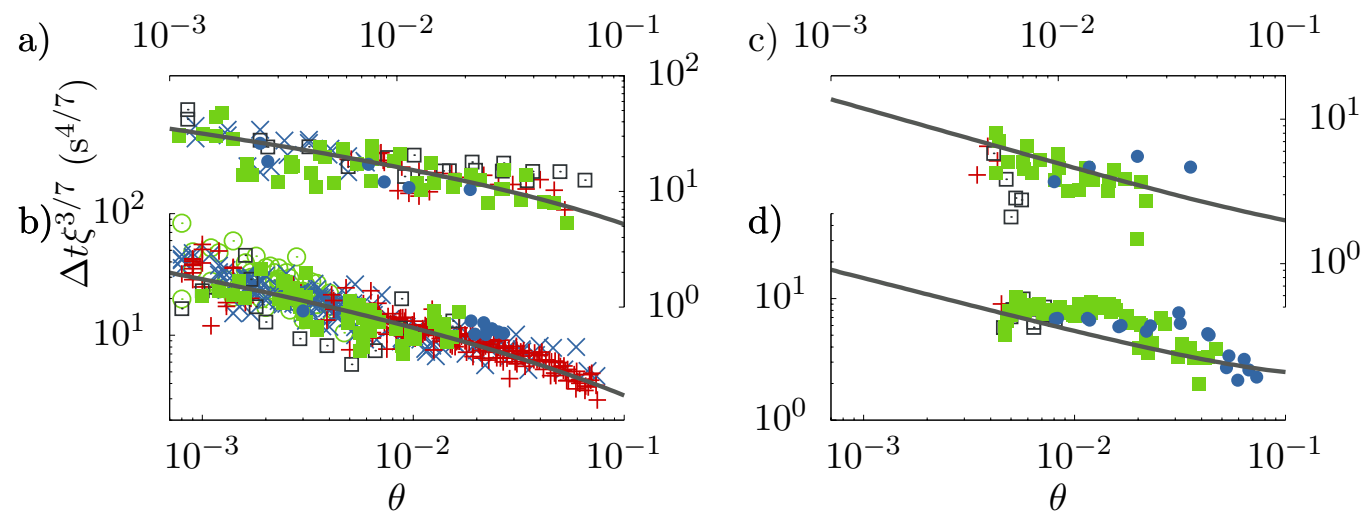

Figure 5.8: Temperature dependence of $\Delta t \xi^{3 / 7}$ for IBE $+\mathrm{W}$ and $\mathrm{M}+\mathrm{H}$ mixtures. Data points are shown for the upper (top) and the lower layer (bottom) of mixtures of IBE $+\mathrm{W}$ (left) and $\mathrm{M}+\mathrm{H}$ (right), respectively. We use the same symbols and colours as in figure 5.1, and show the theoretical prediction, (5.16), by solid lines. The same value of $\alpha$ is found for the upper and the lower layer of the mixtures, $\alpha=0.6$ for IBE $+\mathrm{W}$ (left), and $\alpha=0.9$ for $\mathrm{M}+\mathrm{H}$ (right).

fig. 5.9. The prediction is plotted for $\alpha=0.69$, which describes the data very well. The different value of $\alpha$ is chosen based on a typical $k=2 \times 10^{5}$, which is more representative for the ramp rate of $\xi=2.5 \times 10^{-5} \mathrm{~s}^{-1}$ (see red + ) in fig. 5.7(c).

In summary the model based on the two growth contributions sketched in fig. 5.5 gives rise to the bottleneck time scale, which sets the oscillation period. The experimental data for two types of mixtures and the data, where the influence of stirring was investigated, collapse onto the predicted master curve.

\subsection{The relevance of Ostwald ripening: A quantitative test}

The collapse of the experimental data for the oscillation period in figs. 5.7 and 5.8 and the quantitative agreement with the predictions of our model eqs. (5.15a) and (5.16) allows for a comparison with growth model of Ostwald ripening. I conclude the present chapter by a discussion of the two possible ways of modelling the growth contribution for small droplets. Ostwald ripening (section 5.2.1.2) and the accretion of material provided by the constant driving (section 5.2.1.1) lead to the same prediction of the scaling of the oscillation period $\Delta t$. The difference lies only in the prefactors, which give rise to different values for the oscillation period $\Delta t$ and also for the bottleneck radius $r^{*}$. 


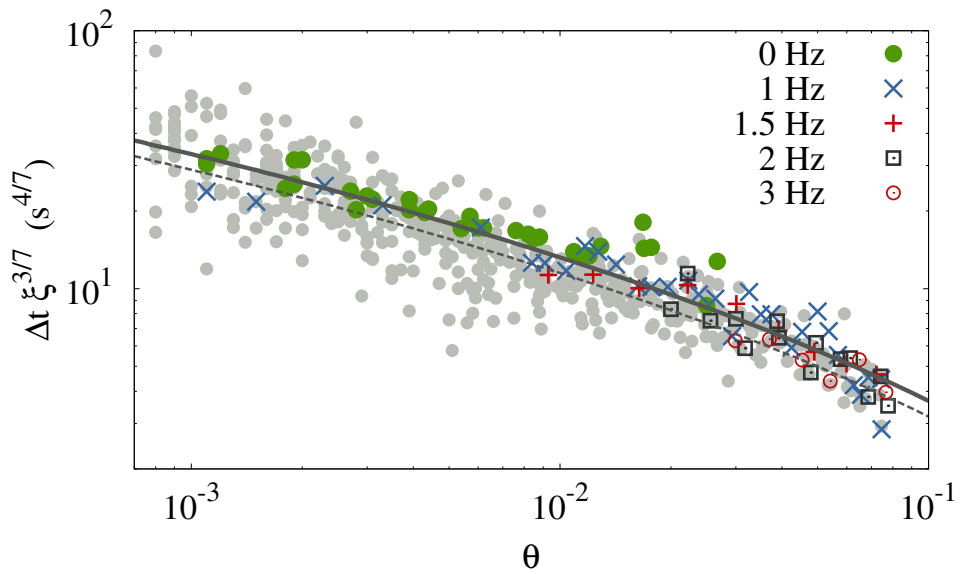

Figure 5.9: Temperature dependence of $\Delta t \xi^{3 / 7}$ for the lower layer of the IBE $+\mathrm{W}$ mixture. The grey dots show the data of fig. 5.8(b). The grey solid line shows the prediction of the model, eq. (5.16), with $\alpha=0.69$ and the dashed line the prediction with $\alpha=0.6$. The coloured symbols show the data points for different stirring rates as indicated in the legend. Here the same data and symbols are used as for fig. 5.3.

\subsubsection{Values of $\alpha$ for binary mixtures}

The solid lines in fig. 5.8 faithfully provide the $\theta$ dependence of $\Delta t$ even though eq. (5.16) only accounts for the temperature dependence of the material constants, and disregards the temperature dependence of $\varepsilon$ and $k$, that should be present according to our discussion of fig. 5.7 in section 5.3.1. Consequently, the dimensionless prefactor $\alpha$ is the only free parameter in eq. (5.16). Typical values of $\varepsilon$ are $0.1 \lesssim \varepsilon \lesssim 1$ (Beard and Ochs, 1993), and $10^{5} \lesssim k \lesssim 10^{7}$ for the IBE $+\mathrm{W}$ system (see fig. 5.7(c)). For $k=5.4 \times 10^{5}$ and $\varepsilon=0.3$ one indeed finds the value $\alpha=0.6$ adopted in fig. 5.8. There is only a minor variability of $\alpha$ in spite of the substantial range of values taken by $\varepsilon$ and $k$ : the $(1 / 7)^{\text {th }}$ and $(3 / 7)^{\text {th }}$ power in eq. (5.16) strongly suppress these dependencies.

In contrast, eq. (5.16) provides $\alpha=4.36$ for Ostwald ripening when adopting $\varepsilon=0.3$, as before, and inserting $\delta \simeq 1 / 2$ for the factor $k$. This prediction for $\Delta t$ is too large by a factor of about seven.

\subsubsection{Bottleneck radius}

Figure 5.10 shows the time evolution of the distribution of the droplet volume fraction $v(r, t)$ of droplets of radius $r$. Panel a) provides an overview in terms of a radius vs. time plot where $v(r, t)$ is indicated by false colour. Each of the panels b) $-\mathrm{d}$ ) shows twelve curves that describe the evolution of the distribution during one oscillation. 

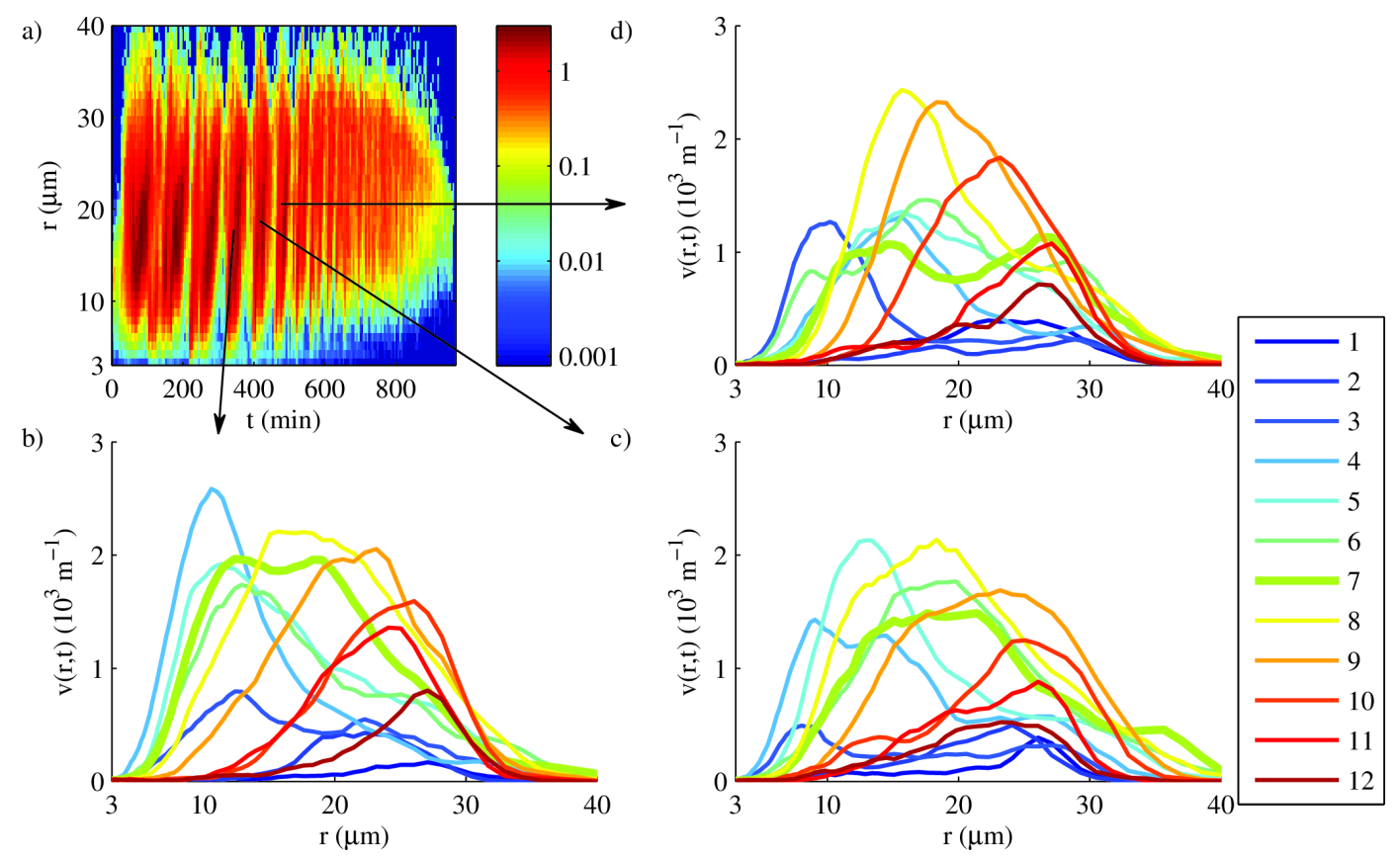

Figure 5.10: Evolution of the droplet size distribution for the lower layer of the IBE $+\mathrm{W}$ mixture subjected to a ramp rate of $\xi=1.05 \times 10^{5} \mathrm{~s}^{-1}$. (a) A radius-time plot of the distribution of the droplet volume fraction $v(r, t)$ clearly captures the oscillations in time. (b)-(d) explicitly provide the radial distribution of the volume fraction for the fourth, fifth and sixth oscillation. To suppress fluctuations the distributions are determined as temporal averages over one of twelve time intervals of equal length in each oscillations. Within each period the distributions at different times are labelled by a colour coding ranging from blue to red, as specified in the legend. The thicker green lines, number 7 , correspond to the time where the bottleneck is crossed.

In the beginning of each period there is a pronounced peak for small radii (blue lines). The maximum of the distribution shifts to larger radii as the distribution evolves, it develops a shoulder (curve 3-5), becomes bimodal (thick green curve 7), and then the number of large droplets rapidly decays (curves 8-12). I attribute the decay to precipitation. The arising of the shoulder reflects the broadening of the distributions when the largest droplets have crossed the bottleneck (Beard and Ochs, 1993; Kostinski and Shaw, 2005). From this perspective the minimum arising in the bimodal droplet spectra should amount to the bottleneck radius, $r^{*}$. For the data of measurements in the lower layer of IBE $+\mathrm{W}$, that are shown in fig. 5.10, the bottleneck radius is thus found to lie in the range $r^{*} \simeq 15 \ldots 20 \mu \mathrm{m}$ [cf. the thick green curves, number 7, in fig. 5.10.b)-d)].

The observed values for the bottleneck radius match exactly with the radius calculated 
based on eq. (5.9). Indeed, for the data shown in fig. 5.7 we find values for $r^{*}$ that decrease from $20 \mu \mathrm{m}$ for small values of $n \kappa^{-3} \xi^{-4}$ to $10 \mu \mathrm{m}$ for the largest considered values. In contrast, when evaluating the bottleneck radius in the case of Ostwald ripening, eq. (5.9), for the lower layer of IBE+W, $\xi=1 \times 10^{-5} \mathrm{~s}^{-1}$ and $\varepsilon=0.3$ one finds values between $0.1 \mu \mathrm{m}$ and $0.8 \mu \mathrm{m}$ for the whole range of reduced temperatures. These values are two orders of magnitude smaller than the values inferred from the experiments, fig. 5.10.

It is also instructive to compare the bottleneck radius $r^{*}$ to the droplet radius, $r_{\mathrm{Pe}}$, where the Pèclet number, Pe, of the droplet motion crosses one. Calculating $\mathrm{Pe}=u L / D_{d}$ based on the sedimentation velocity $u=2 \Delta \rho g r_{\mathrm{Pe}}^{2} /(9 \eta)$, the droplet diameter $L=2 r_{\mathrm{Pe}}$, and the Brownian droplet diffusivity $D_{d}=k_{B} T /\left(6 \pi \eta r_{\mathrm{Pe}}\right)$ yields

$$
\mathrm{Pe}=\frac{8 \pi}{3} \frac{\Delta \rho g}{k_{B} T} r_{\mathrm{Pe}}^{4}
$$

It takes values $\mathrm{Pe} \simeq 1$ for droplet radii $r_{\mathrm{Pe}}=\left[k_{B} T /(8 \pi \Delta \rho g)\right]^{1 / 4} \simeq 1.2 \mu \mathrm{m}$. In line with expectation, the prediction for the bottleneck radius $r^{*}$ is of the same order of magnitude, even though somewhat larger than $r_{\mathrm{Pe}}$, when properly accounting for the contribution to the initial droplet growth by the constant ramp rate. In contrast, they are predicted to be up to one order of magnitude smaller than $r_{\mathrm{Pe}}$, when only accounting for Ostwald ripening. These severe discrepancies between observations and predictions rule out Ostwald ripening as a relevant contribution to growth in our experiments.

\subsubsection{Temperature dependence of $\Delta t$}

In contrast to a suggestion in the literature (Wilkinson, 2014) I am reluctant to attribute the $\theta$ dependence of $\Delta t \xi^{3 / 7}$ to the critical scaling of the material constants entering eq. (5.16), i.e. the dependence on $D, \sigma=2 \gamma V_{m}^{2} C_{\infty} /(R T)$ and $\kappa=2 g \Delta \rho /(9 \mu)$. The reason is fourfold:

(i) the values of $\theta$ in the experiments clearly lie outside the critical range. This is documented in the appendix where I report a much more involved $\theta$ dependence of the material constants than the power-law singularities describing the scaling for small reduced temperatures $\theta$;

(ii) in addition to $D, \sigma$ and $\kappa$ also the collection efficiency $\varepsilon$ shows a noticeable temperature dependence, as observed in fig. 5.7(a) and (b);

(iii) the parameter $k$ entering the definition, eq. (5.16), of $\alpha$ has a noticeable temperature dependence (see fig. 5.7(c));

(iv) for the mixtures under consideration the dependence of $\varepsilon$ and $k$ cancels partially. 
Consequently, the close correspondence of the $\theta$ dependence of the prediction eq. (5.16), and the one obtained by considering $\alpha$ to be a constant and $D, \sigma$ and $\kappa$ to vary according to the power laws valid very close to critical point might very well be a coincidence. A proper discussion of the temperature dependence of $\Delta t$ should first address the intriguing observation that $k$ takes surprisingly large values in the present experiments, and that the observed values vary so little that their dependence need not be considered to obtain a good estimate of the oscillation period, fig. 5.8.

\subsection{Summary}

In this chapter a low dimensional model has been developed for the oscillation period of episodic precipitation in binary mixtures. Spatial degrees of freedom were not addressed because of sufficient inherent mixing of fluid volume due to convection. The strength of the temperature ramp is characterized by the dimensionless parameter $k-1$ that amounts to the ratio of droplet volume growth in response to the temperature change and the growth on expense of other, smaller droplets (Ostwald ripening). In our experiments $k$ takes very large values: $k \gtrsim 10^{5}$. Thus, the temperature ramp decouples the evolution equation of droplet sizes, such that the model for the oscillation period is left with an effective radius describing the largest droplets in the system. As a consequence, the two growth mechanisms, that are developed at the level of size distributions in the previous chapter, can be simplified to give an analytically treatable model. The model suggests a master plot that facilitates a data collapse of all of our experimental data, and it provides a theory curve that describes the data without adjustable parameters. 



\section{Periodic Driving}

In this chapter I explore the impact of periodic driving $\xi(t)$ with $\xi\left(t+\Delta t_{\text {ext }}\right)=\xi(t)$ on phase separation in binary mixtures. On the one hand many processes where continuously driven phase separation plays a role show fluctuations or even large scale convection, e.g. in clouds that produce variations in the driving. On the other hand, the description of systems with such a more complicated driving provides a test of the model for episodic precipitation: Can it describe also dynamic features like phase locking, with respect to the period of the driving?

The chapter has two parts. I start with the model for episodic precipitation that has been established in chapter 5 and study the impact of the oscillatory driving. Subsequently, I report on measurements where the oscillatory driving has been realized experimentally, and compare the observations to the predictions of the model.

\subsection{Phase locking and Arnold tongues}

In the present section we establish a model for the oscillation period of oscillatory demixing with time-periodic driving,

$$
\xi(t)=\xi_{\mathrm{av}}\left[1+A \pi_{0}(t)\right] .
$$

The form of the perturbation is described by the function $\pi_{0}(t)$ that is period in time with period $\Delta t_{\text {ext }}$. The time averaged driving is $\xi_{\text {av }}$, and the relative strength of the driving is controlled by the amplitude $A$.

\subsubsection{The state variables and their evolution equations}

I start with recalling the model for constant driving $\xi$, see eq. (5.7)

$$
\dot{r}=\frac{\xi}{4 \pi n} \frac{1}{r^{2}}+\frac{\varepsilon \kappa \xi t}{4} r^{2} .
$$

In the following I use the model formulated in terms of droplet volume $v=\frac{4 \pi}{3} r^{3}$. The term $\xi t$ in the second contribution to eq. (6.2) has to be generalized to $\phi(t)$, such that its time derivative $\dot{\phi}(t)$ equals $\xi(t)$. Consequently, we have two variables $v(t)$ and $\phi(t)$ that characterize the state of periodically driven episodic precipitation. 
They obey the following evolution equations

$$
\begin{aligned}
& \dot{v}(t)=4 \pi r^{2}(t) \dot{r}(t)=\frac{\xi(t)}{n(t)}+\left(\frac{3^{4}}{4^{4} \pi}\right)^{1 / 3} \varepsilon \kappa \phi(t) v^{4 / 3}(t), \\
& \dot{\phi}(t)=\xi(t) .
\end{aligned}
$$

\subsubsection{Dimensionless units}

For further investigations we use a dimensionless form of eq. (6.3). We measure time in units of $\Delta t_{\text {ext }}$, and the number density and volumes in terms of a characteristic density $n_{0}$ that is chosen such that $\xi_{\text {av }} \Delta t_{\text {ext }} /\left[n\left(t=t_{0}\right) / n_{0}\right]=1$ at the beginning of integration, $t=t_{0}$. The evolution equations 6.3 for the non-dimensionalised quantities reduce then to

$$
\begin{aligned}
\dot{v} & =\xi(t)+3 \alpha \phi(t) v^{4 / 3}(t), \\
\dot{\phi} & =\xi(t), \\
\text { with } \quad \xi(t) & =1+A \pi_{0}(t) \\
\alpha & =\left(\frac{3}{4^{4} \pi}\right)^{1 / 3} \frac{\varepsilon \kappa \xi_{\mathrm{av}} \Delta t_{\mathrm{ext}}^{2}}{n_{0}^{1 / 3}} .
\end{aligned}
$$

Here, the volume fraction $\phi$ has been augmented with a factor $\xi_{\text {av }} \Delta t_{\text {ext }}$ in order to avoid non-trivial factors in eq. (6.4b). Moreover, the factor $n$ has been suppressed in the denominator of the $\xi$ term in eq. (6.4a). This is admissible, because the number density $n$ is preserved as long as there is only diffusive growth, i.e. as long as the first term dominates the right hand side of eq. (6.4a). At later times, when the growth is dominated by collection of small droplets, the second contribution dominates, and the omission does not matter. Altogether, one thus arrives at eqs. (6.4b) and (6.4c) with an average driving of unit strength. In the remainder of this section we integrate eq. (6.4) based on the approximation introduced in section 5.2.2 to obtain the parameter dependence of the oscillation period $\Delta t(A=0)$. Here, this will allows us to obtain analytic expressions for $\Delta t(A)$ with $A \neq 0$. In section 6.1.5, I compare the resulting theoretical predictions with the results of numerical integration.

\subsubsection{Oscillation period for $A=0$}

For constant driving the oscillation period can faithfully be described as the time scale needed of droplets of zero size, $v_{0}=0$, to reach infinite size. For $A=0$ we may 
start integrating at time $t_{0}=0$, because the phase of the external driving does not matter.

For small droplets the first contribution dominates $\dot{v}$ such that $v(t) \simeq \phi(t)=t$. For $1=\xi=3 \alpha \phi\left(t_{c}\right) v^{4 / 3}\left(t_{c}\right) \simeq 3 \alpha t_{c}^{7 / 3}$, there is a crossover to growth dominated by the second term of eq. (6.4a). Consequently, for times $t>t_{c}=v_{c}$, we find

$$
v^{-1 / 3}=v_{c}^{-1 / 3}-\alpha \int_{t_{c}}^{t} \phi(t) \mathrm{d} t=t_{c}^{-1 / 3}-\frac{\alpha}{2}\left(t^{2}-t_{c}^{2}\right) .
$$

At time $t=p$, i.e. after one period of the cycle, the volume diverges, and $v^{-1 / 3}$ approaches zero. Consequently, the parameter $\alpha$ is connected to the oscillation period, $p$, of the unperturbed dynamics via ${ }^{1}$

$$
\alpha=\frac{1}{3}\left(\frac{7}{p^{2}}\right)^{7 / 6},
$$

where the crossover relation $1=3 \alpha t_{c}^{7 / 3}$ is used to eliminate $t_{c}$ in eq. (6.5).

Next, we explore how the period changes upon varying $A$ for settings with different $p$.

\subsubsection{Oscillation period for $A>0$}

In our experiments we switch the driving between two different ramp rates, $\xi_{\max }$ and $\xi_{\min }$. This amounts to a driving with average ramp rate $\xi_{\mathrm{av}}=\left(\xi_{\max }+\xi_{\min }\right) / 2$, amplitude $A=\left(\xi_{\max }-\xi_{\min }\right) /\left(2 \xi_{\text {av }}\right)$, and a shape $\pi_{0}(t)$ that takes the form of a

${ }^{1}$ The relation between $\alpha$ and $p$ in eq. (6.6) in the present notation is exactly the parameter dependence of the oscillation period $\Delta t$, derived in eq. (5.15b) of the previous chapter. This can be seen by recalling that $p=\Delta t(A=0) / \Delta t_{\text {ext }}$. Using eqs. (6.4d) and (6.6) we write

$$
\frac{\Delta t}{\Delta t_{\mathrm{ext}}}=p=\sqrt{7}(3 \alpha)^{-3 / 7}=\sqrt{7}\left(\frac{4^{4} \pi}{3^{4}} \frac{n_{0}}{\left(\varepsilon \kappa \xi_{\mathrm{av}}\right)^{3} \Delta t_{\mathrm{ext}}^{6}}\right)^{1 / 7} .
$$

With the choice of $n_{0}$ such that $n_{0} \Delta t_{\text {ext }}=n / \xi_{\text {av }}$ one obtains

$$
\Delta t=\sqrt{7}\left(\frac{4^{4} \pi}{3^{4}} \frac{n}{(\varepsilon \kappa)^{3} \xi_{\mathrm{av}}^{4}}\right)^{1 / 7} .
$$

This matches exactly the expression of eq. (5.15b), when the $\sqrt{7}$ coming from the analytically treatable approximation, that has been adopted in the present chapter, is replaced by the prefactor 2.44 which is obtained by numerical integration of the full equation. After all, $2.44\left(4^{4} \pi / 3^{4}\right)^{1 / 7}=3.39$. 


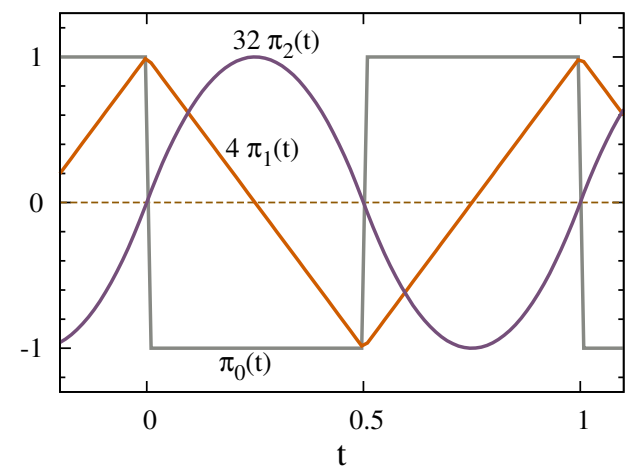

Figure 6.1: Functions characterizing the periodic driving. The piecewise constant function $\pi_{0}(t)$ (gray, eq. (6.7)), piecewise-linear function $4 \pi_{1}(t)$ (orange, eq. (6.9)) and piecewiseparabolic function $32 \pi_{2}(t)$ (violet, eq. (6.11)).

square-wave function (see fig. 6.1),

$$
\pi_{0}(t)= \begin{cases}-1 & \text { for } 0 \leq \tau<\frac{1}{2} \\ +1 & \text { for } \frac{1}{2} \leq \tau<1\end{cases}
$$

where $\tau$ is here the fractional part of $t$.

We start integrating at time $t_{0}$ with zero droplet volume fraction, $\phi\left(t=t_{0}\right)=0$. According to eq. (6.4b) the volume fraction takes the following form at later times,

$$
\phi(t)=t-t_{0}+A\left[\pi_{1}(t)-\pi_{1}\left(t_{0}\right)\right] .
$$

Here $\pi_{1}(t)$ is the periodic, piecewise-linear function with zero mean and $\dot{\pi}_{1}(t)=\pi_{0}(t)$ that is also shown in fig. 6.1,

$$
\pi_{1}(t)= \begin{cases}\frac{1}{4}-t & \text { for } 0 \leq \tau<\frac{1}{2} \\ -\frac{3}{4}+t & \text { for } \frac{1}{2} \leq \tau<1\end{cases}
$$

Finally, for the solution of eq. (6.4a) we will need the time integral of $\phi(t)$. For the present driving it takes the form

$$
\int_{t_{c}}^{t} \phi(t) \mathrm{d} t=\frac{1}{2}\left(t^{2}-t_{c}^{2}\right)-\left[t_{0}+A \pi_{1}\left(t_{0}\right)\right]\left(t-t_{c}\right)+A\left[\pi_{2}(t)-\pi_{2}\left(t_{c}\right)\right],
$$


which involves the piecewise parabolic function

$$
\pi_{2}(t)= \begin{cases}\frac{1}{2} t\left(\frac{1}{2}-t\right) & \text { for } 0 \leq \tau<\frac{1}{2} \\ \frac{1}{2}\left(t-\frac{1}{2}\right)(t-1) & \text { for } \frac{1}{2} \leq \tau<1 .\end{cases}
$$

In analogy to the case for $A=0$ the evolution of $v(t)$ proceeds in two steps. Integration of eq. (6.4a) from zero droplet volume at time $t_{0}$ we find the critical size $v_{c}$ for the crossover to the regime where growth is dominated by collection,

$$
v_{c}=\phi\left(t_{c}\right)=t_{c}-t_{0}+A\left[\pi_{1}\left(t_{c}\right)-\pi_{1}\left(t_{0}\right)\right],
$$

as well as the crossover condition $\xi\left(t_{c}\right)=3 \alpha v_{c}^{7 / 3}$, i.e.

$$
v_{c}=\frac{p}{\sqrt{7}}\left[1+A \pi_{0}\left(t_{c}\right)\right]^{3 / 7} .
$$

Subsequent integration for large droplets yields

$$
\frac{2}{\alpha} v^{-1 / 3}(t)=\frac{2}{\alpha} v_{c}^{-1 / 3}-\left[t-t_{0}-A \pi_{1}\left(t_{0}\right)\right]^{2}+\left[v_{c}-A \pi_{1}\left(t_{c}\right)\right]^{2}-2 A\left[\pi_{2}(t)-\pi_{2}\left(t_{c}\right)\right] .
$$

For $v^{-1 / 3}\left(t_{1}\right)=0$ this can be solved for $t_{1}$,

$$
\begin{aligned}
t_{1}=t_{0}+A \pi_{1}\left(t_{0}\right)+\left[\frac{2}{\alpha} v_{c}^{-1 / 3}+\left[v_{c}-A \pi_{1}\left(t_{c}\right)\right]^{2}-2 A\left[\pi_{2}\left(t_{1}\right)-\pi_{2}\left(t_{c}\right)\right]\right]^{1 / 2} \\
=t_{0}+A \pi_{1}\left(t_{0}\right)+\left[p^{2}\left[1+A \pi_{0}\left(t_{c}\right)\right]^{-1 / 7}\left(1+\frac{A}{7} \pi_{0}\left(t_{c}\right)\right)\right. \\
\left.\quad-2 A\left(\pi_{2}\left(t_{1}\right)-\pi_{2}\left(t_{c}\right)+\frac{p}{\sqrt{7}} \pi_{1}\left(t_{c}\right)\left[1+A \pi_{0}\left(t_{c}\right)\right]^{3 / 7}\right)+A^{2} \pi_{1}^{2}\left(t_{c}\right)\right]^{1 / 2}
\end{aligned}
$$

with $\quad t_{c}=t_{0}+\frac{p}{\sqrt{7}}\left[1+A \pi_{0}\left(t_{c}\right)\right]^{3 / 7}-A\left[\pi_{1}\left(t_{c}\right)-\pi_{1}\left(t_{0}\right)\right]$

In principle the set of equations eq. (6.13) can be solved for $t_{1}$ because the functions $\pi_{2}\left(t_{1}\right)$, is piecewise parabolic. However, for the purpose of identifying fixed points of the dynamics it is sufficient to consider cases where $t_{1}=t_{0} \bmod 1$, and the $A$ and $p$ dependence of these fixed points is faithfully described by the leading order 

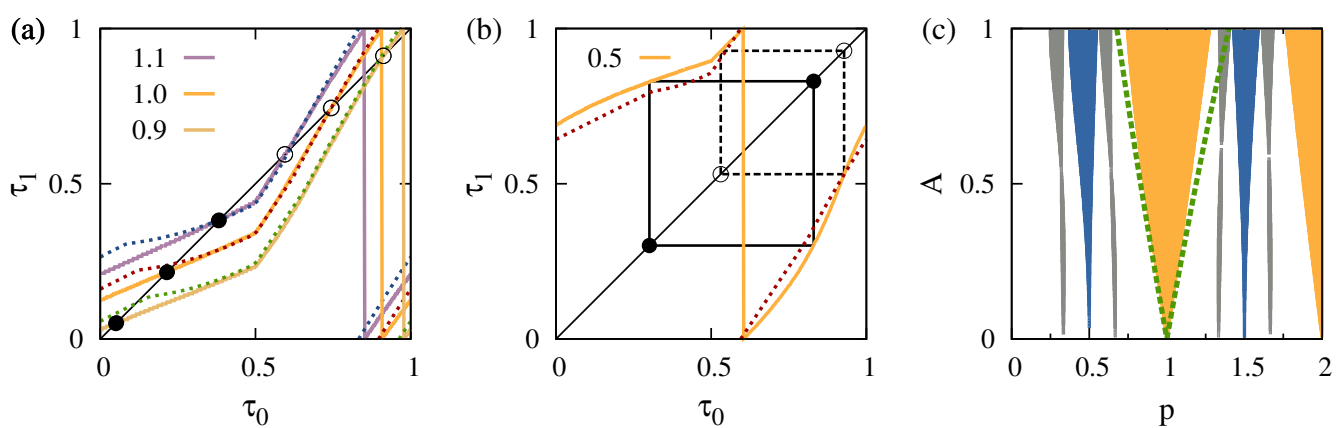

Figure 6.2: Phase maps and Arnold tongues. (a) Phase map for $A=0.5$ and three different values of detuning, $p=0.9,1.0$, and 1.1. The filled and open circles mark stable and unstable fixed points, respectively. (b) Phase map for $A=0.5$ and $p=0.5$. The stable and unstable period-two cycles are marked by solid and broken black lines respectively. In panel (a) and (b) the solid lines mark numerical results, and the corresponding dotted lines are theoretical curves based on eq. (6.14). (c) The Arnold tongues represent a phase diagram indicating for which combinations of $A$ and $p$ one encounters stable fixed points (regions with locking to an integer value of $p$ ), period-two cycles (regions with locking to half integer values of $p$ ), and period-three cycles (regions with locking to values $p=j / 3$ where $j$ is no multiple of three). The green dashed lines bounding the 1:1 Arnold tongue are theoretical predictions, eq. (6.15). Numerical data, where we found period 1, 2 and 3 cycles are marked by orange, blue, and grey dots, respectively.

approximation of eq. (6.13),

$$
\begin{aligned}
t_{1}\left(t_{0}\right) & \simeq t_{0}+p+A\left[\pi_{1}\left(t_{0}\right)-\frac{\pi_{1}\left(t_{c}\right)}{\sqrt{7}}-\frac{\pi_{2}\left(t_{0}\right)-\pi_{2}\left(t_{c}\right)}{p}\right] \\
\text { with } t_{c} & =t_{0}+\frac{p}{\sqrt{7}} .
\end{aligned}
$$

The resulting expressions for $p=1$ and $p=1 / 2$ are plotted by broken lines in fig. 6.2(a) and (b), respectively. They nicely agree with the numerical results that are provided by solid lines.

\subsubsection{Phase locking and Arnold tongues}

The plots, fig. 6.2(a),(b), of the fractional part of $t_{1}$ as function of $t_{0}$ provide the phase map for the evolution of the oscillations. Iterating the map, describes the evolution of the phase where precipitation arises with respect to the external driving.For values of $p$ close to 1 the phase converges towards a stable fixed point in the interval $\left[0 ; \frac{1}{2}\right]$, fig. 6.2(a). The presence of this fixed point indicates, that episodic precipitation matches with the period of the external driving, and that the phase of the oscillations 
are locked at the value prescribed by the stable fixed point of the phase map. Figure 6.2(b) shows a situation where there are two cycles of episodic precipitation in each period of the external driving. This amounts the region in parameter space where the phase map has a stable period-two cycle. The parameters, $(p, A)$, where there are $j$ cycles of precipitation in $i$ periods of the external driving are denoted as the $i: j$ Arnold tongue (Pikovsky et al., 2003). In fig. 6.2(c) we show the $1: 1$, the $1: 2$, the $1: 3$ and the $2: 3$ Arnold tongues for episodic precipitation with the time-periodic driving described by eqs. (6.1) and (6.7).

Let us take a closer look at the $1: 1$ Arnold tongue and its phase maps, fig. 6.2(a). Equation (6.14) reveals, that to first order the parameter $p$ results in a vertical displacement of the phase map. Consequently, when $p$ is increased, the fixed point moves to the right, until it collides with its unstable partner at $\tau=\frac{1}{2}$. In contrast, the fixed point moves to the left when $p$ is decreased, until it collides with its unstable partner at $\tau=0$. For each amplitude $A>0$ one thus determines the interval $\left[p_{1: 1}^{l}(A) ; p_{1: 1}^{r}(A)\right]$ where the phase map has a stable fixed point. Equation (6.14) also reveals that the amplitude of the undulations in the phase map, i.e. the maximal deviation from a linear function, is proportional to $A$. Consequently, the interval $\left[p_{1: 1}^{l}(A) ; p_{1: 1}^{r}(A)\right]$ shrinks to the point $p=1$ for $A=0$, and it grows monotonically when $|A|$ is increased. The boundaries of the interval can be determined by observing that the saddle-node bifurcations where the stable and the unstable fixed points collide arise at $t_{0}=0$ and $t_{0}=1 / 2$. Solving eq. (6.14) for the according $p(A)$ dependence with $t_{0}=0$ and $t_{1}=1$ for the left and $t_{0}=1 / 2$ and $t_{1}=3 / 2$ for the right boundary of the 1:1 Arnold tongue leads to

$$
\begin{aligned}
& p_{1: 1}^{l}=\frac{1-\left(\frac{1}{4}-\frac{1}{8 \sqrt{7}}\right) A}{1+\frac{3}{28} A} \\
& p_{1: 1}^{r}=\frac{1+\left(\frac{1}{4}-\frac{1}{8 \sqrt{7}}\right) A}{1-\frac{3}{28} A} .
\end{aligned}
$$

The expressions only differ by a change of the sign of $A$, due to the symmetry of the equations of motion with respect to shifting $t$ by half a period and reversing the sign of $A$. They nicely describe the boundary of the $1: 1$ Arnold tongue for amplitudes $A<0.5$, see fig. $6.2(\mathrm{c})$. The saddle-node bifurcations marking the borders of the $1: 2$ Arnold tongues also arise at $t_{0}=0$ and $t_{0}=1 / 2$, respectively. Hence, one can evaluate eq. (6.14) to determine the second return time $t_{2}\left(t_{1}\left(t_{0}\right)\right)$, and demand that $t_{2}\left(t_{1}(0)\right)=0$ in order to find the boundaries of the $1: 2$ Arnold tongue. This will be discussed in more detail in forthcoming work. 


\subsection{Experimental results}

This section deals with the experimental observations of synchronisation in periodically driven phase separation in mixtures of IBE $+\mathrm{W}$. I start with the experimental realization of temperature ramps that produce approximately a square-wave driving. Subsequently I show data for period 1-cycles, period 2-cycles and period 3-cycles, followed by observations for $p=5$, where the external driving period is only a small fraction of the undisturbed period.

\subsubsection{Realization of temperature ramps}

The temperature ramps are calculated based on eq. (2.9). One challenge is to deal with the decreasing of the oscillation period during a measurement, observed in fig. 2.6. Typically the period of the undisturbed oscillator is fixed and it is easy to define the period of the external forcing. In our case we also have to keep the ratio of the external period and the period of the undisturbed oscillation constant, when we want to study synchronisation. Hence, the period of $\xi(t)$ has to decrease in the same way as $\Delta t$ does. This is achieved by calculating the temperature ramps in such a way, that the period of the external driving $\Delta t_{\text {ext }}$ is adjusted to be $\Delta t_{\text {ext }}=\Delta t(\bar{T}) / p$, where $\bar{T}$ is the mean temperature of $\Delta t_{\text {ext }}$. This is done iteratively during the numerical integration of eq. (2.9) with the condition, that $\Delta t_{\text {ext }, n+1}=\Delta t(\bar{T}) / p$ and $\bar{T}\left(\Delta t_{n}\right)$. All experimental runs are done with an average driving ${ }^{2}$ of $\xi_{\mathrm{av}}=2 \times 10^{-5} \mathrm{~s}^{-1}$. They start at $T_{0}=25.8^{\circ} \mathrm{C}$ and end at $50^{\circ} \mathrm{C}$. For these parameters the iteration typically converges after three iteration steps.

In fig. 6.3 three experimental realizations of the periodic driving are shown for $p=1$ and $A=0.5$. The ramp rate $\xi(t)$ is calculated according to section 2.3.4 from the temperature log files of the respective measurements. The $\xi$-values reflect the fluctuations in the temperature and do not show a clear step function jumping between $\xi_{\min }$ and $\xi_{\max }$. On the other hand the determination of the ramp rate from the fluctuating temperature involves a numerical derivative. This has been calculated by total-variation regularization (Chartrand, 2011), to avoid noise amplification. However, the correct quantification of the fluctuations in the derivative of noisy data is very sophisticated, such that one should not put too much weight into the fluctuations of the given $\xi$-values. The realization of the driving is sufficiently good for the purpose of testing the robustness of the theory with respect to a time-periodic and noisy driving, $\xi(t)$.

The start of the low $\xi$-regions, which will be used as a reference time to define the phase of the oscillations, is obtained by a time average of the $\xi$-values in a small vicinity of $\xi_{\mathrm{av}}$.

\footnotetext{
${ }^{2}$ Here the scaling representation of the phase diagram is used to calculate the temperature ramps.
} 


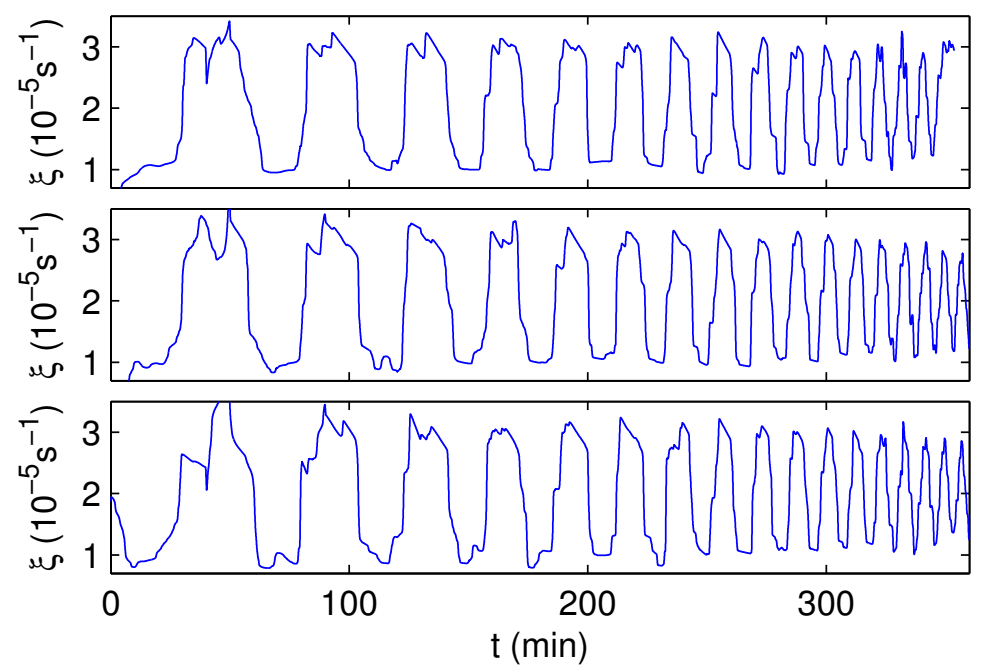

Figure 6.3: Ramp rates $\xi(t)$ for a periodic driving of $A=0.5$ and $p=1$. The different panels show three realisations of the same temperature ramp. The ramp rate has been determined from the recorded temperature during measurement, see section 2.3.4.

\subsubsection{1:1 Arnold tongues}

For $p=1$ the external driving period matches the period of the undisturbed oscillations. The oscillation periods are determined from turbidity measurements as described in section 2.4. From the space-time plots the oscillations are extracted from a horizontal line in the lower part of the lower layer, see fig. 6.4(left).

The next step towards the observation of synchronisation is to analyse the phases of the two oscillating signals: the oscillations in the ramp rate $\xi$ and the turbidity. In fig. 6.4(right) both are plotted on top of each other. After two or three oscillations the peaks in the turbidity lie always at the position, where the ramp rate changes from a high value to a low one. This is already a good indication for synchronisation. To make this observation more quantitative, the positions of the turbidity peaks are determined relative to the beginning of a low- $\xi$ region and normalised to the length of the respective period of the driving. The resulting values $\tau \in[0,1]$ are denoted as the phase of the turbidity. One yields a phase map, when all phases are plotted as a function of the previous phase $\tau_{i+1}\left(\tau_{i}\right)$, where $\tau_{i+1}$ denotes the phase following $\tau_{i}$, see fig. 6.5. In a phase map a 1:1 phase locking, corresponding to a period-1 cycle, shows up if the data points lie only in one spot. This is the case for all measurements shown in fig. 6.5.

In figure fig. 6.5(b) experimental data for $p=1$ is shown together with the phase map and the fixed points predicted by the model. The data points are concentrated 

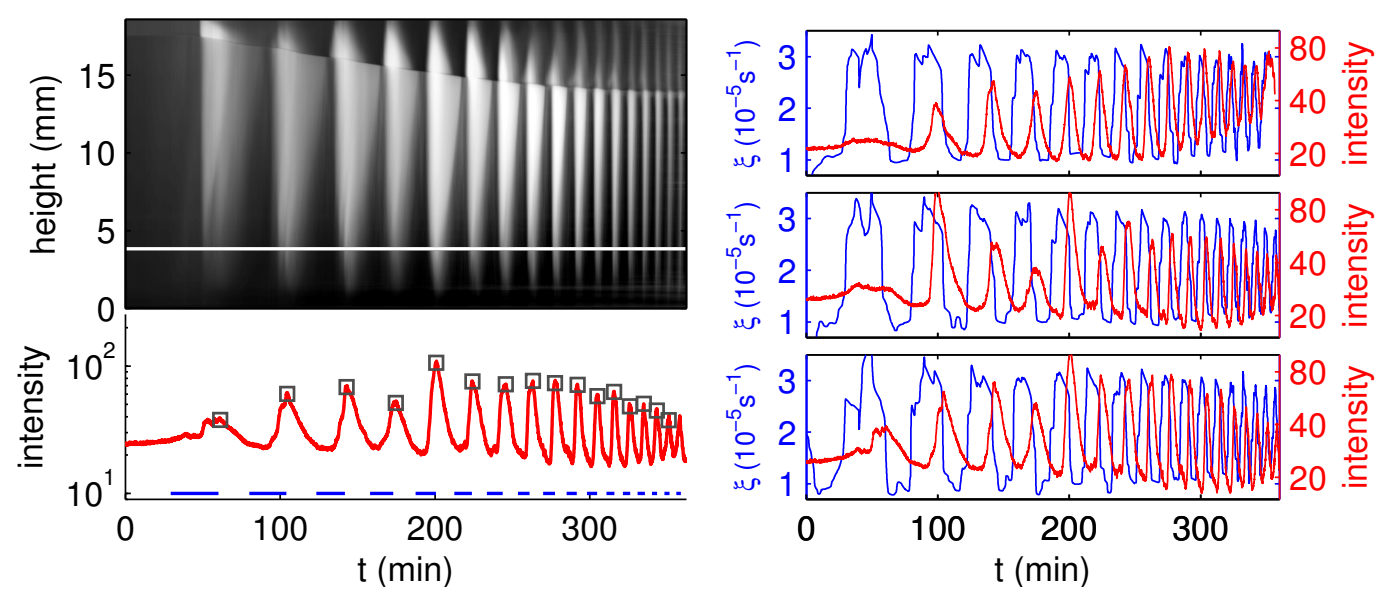

Figure 6.4: Experiments for $p=1$ and $A=0.5$. (Left) Space-time plot of the turbidity and the corresponding intensity (red line) at a height of $4 \mathrm{~mm}$ above the bottom of the measurement cell, as indicated by the white line in the space-time plot. The detected maxima are marked with a gray square. The interrupted blue line at the bottom of the intensity plot indicates the times where the ramp rate has the value $\xi_{\max }$. (Right) The intensity for the three different realisations of the temperature ramp (see fig. 6.3). The ramp rate $\xi(t)$ is shown by blue lines as in fig. 6.3 together with the induced oscillation in the turbidity (red lines) determined from the respective space-time plots. The lower panel corresponds to the space-time plot shown in the left panel.

in the region of the stable fixed point. This agreement is achieved by shifting all the phases by a constant offset $\Delta t_{\text {shift }}=0.15$. In that sense this figure is used to determine the free parameter, that arises when comparing the experiments with the model. The model is based on the evolution of one typical droplet describing the oscillations. One period is over if the radius diverges. On the other hand the turbidity in the experiments is caused by a distribution of droplets. Fixing $\Delta t_{\text {shift }}$ to the value $\Delta t_{\text {shift }}=0.15$ indicates that the peaks in the turbidity appear a little bit before the droplet radius in the model diverges. For the following measurements this free parameter is fixed to $\Delta t_{\text {shift }}=0.15$ and all phases shown in the phase maps are shifted by this value.

In the following I discuss the position of the fixed point for a constant amplitude $A=0.5$ but a varying period ratio $p$ within the borders of the 1:1 Arnold tongue. The position varies from 0 at the left border of the Arnold tongue to 0.5 at the right border where the stable and unstable fixed point meet. In figure fig. 6.5(a) and (c) the phase values for the intermediate cases of $p=0.9$ and $p=1.1$ are shown together with two measurements, respectively. The data is noisy, especially for $p=1.1$. However, one can clearly see the predicted trend that the fixed point moves to the right as $p$ is increased. 

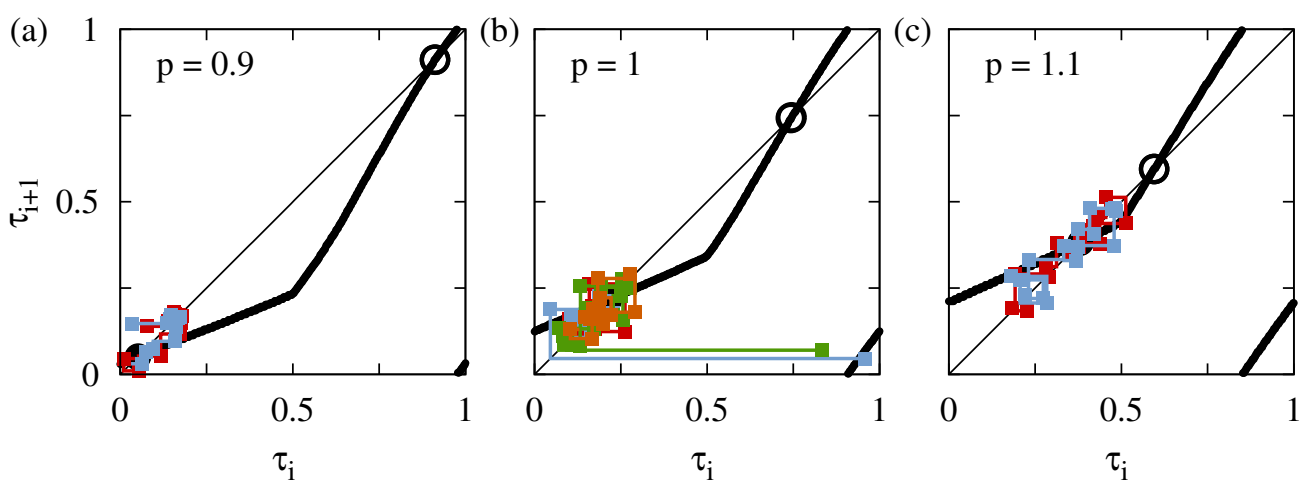

Figure 6.5: Phase maps for $A=0.5$ and (a) $p=0.9$, (b) $p=1$ and (c) $p=1.1$, respectively. The different colors in each panel denote different experimental realisations. The thick black line shows the prediction of the model, the open circle denote the unstable fixed point and the filled circle the stable fixed point (it is hidden by the data points).

\subsubsection{1:2 Arnold tongues}

To study the behaviour of the 1:2 Arnold tongue we analyse measurements for $p=0.5$. The resulting space-time plot is shown in fig. 6.6(a). The turbidity arises in pairs of peaks where a big one is followed by a smaller one. Since the time to develop is shorter for the second peak, not so much droplet volume can be created (the droplet volume fraction increases linear in time) and it therefore has a smaller turbidity.

In the phase map fig. 6.6(c) the period-2-cycle is clearly visible. The data are again shifted by 0.15 and we observe a good agreement for the dominant peak at $\tau \approx 0.8$ with the model predictions. In contrast, the small peak deviates from the predicted value of the model, and it is severely affected by fluctuations. We attribute this to the difficulty in the detection of the small peaks. Because the second peak is small it is very difficult to detect in some measurements. For this reason Vogel (2013) reported that it appears that the period had doubled.

\subsubsection{1:3 Arnold tongues}

To study the behaviour of the 1:3 Arnold tongue we perform measurements with $p=1 / 3$ and $A=0.5$. We observe a reproducible synchronisation of the dominant peak in the turbidity with the beginning of the high- $\xi$ regions, see fig. 6.7(a). The dominant peak is followed by two smaller peaks, which are sometimes hard to detect and not possible to resolve at the end of the measurement. No quantitative comparison is feasible. 

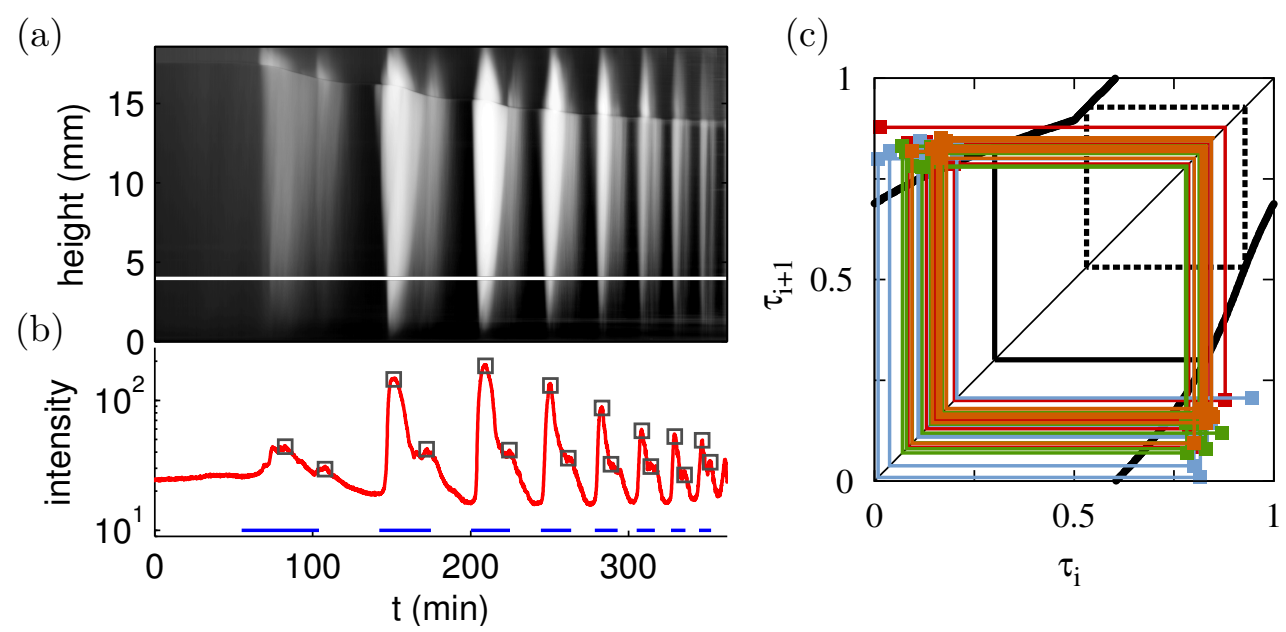

Figure 6.6: Experimental data for the 1:2 Arnold tongue. (a) Space-time plot and the (b) corresponding oscillations for a measurement of $p=0.5$ and $A=0.5$. The oscillating turbidity is obtained by cutting the space-time plot at the white line. The grey squares mark the detected peaks of the turbidity. (c) Phase map for four measurements of $p=0.5$ and $A=0.5$. The thick solid black line is the predicted curve by the model with a stable (solid) and unstable (dashed) period-2-cycle.

\subsubsection{5:1 Arnold tongues}

To explore the impact of a high $p$ value, I also show here a space-time plot for $p=5$, see fig. 6.7(b). In this case, where the external driving changes its value five times in one oscillation period, the system averages over the perturbations. It shows the same behaviour as for constant driving, see fig. 2.6.

\subsection{Summary}

In summary our model for episodic precipitation has been successfully extended to periodic variations in the driving: the resulting predictions agree with experimental observations. The predictions are derived by numerical analysis and complemented by an approximate formulation that is treated analytically. For a small ratio of the external and undisturbed periods, $p$, we observe phase locking, whereas the system only feel the average driving if $p \gtrsim 5$. 

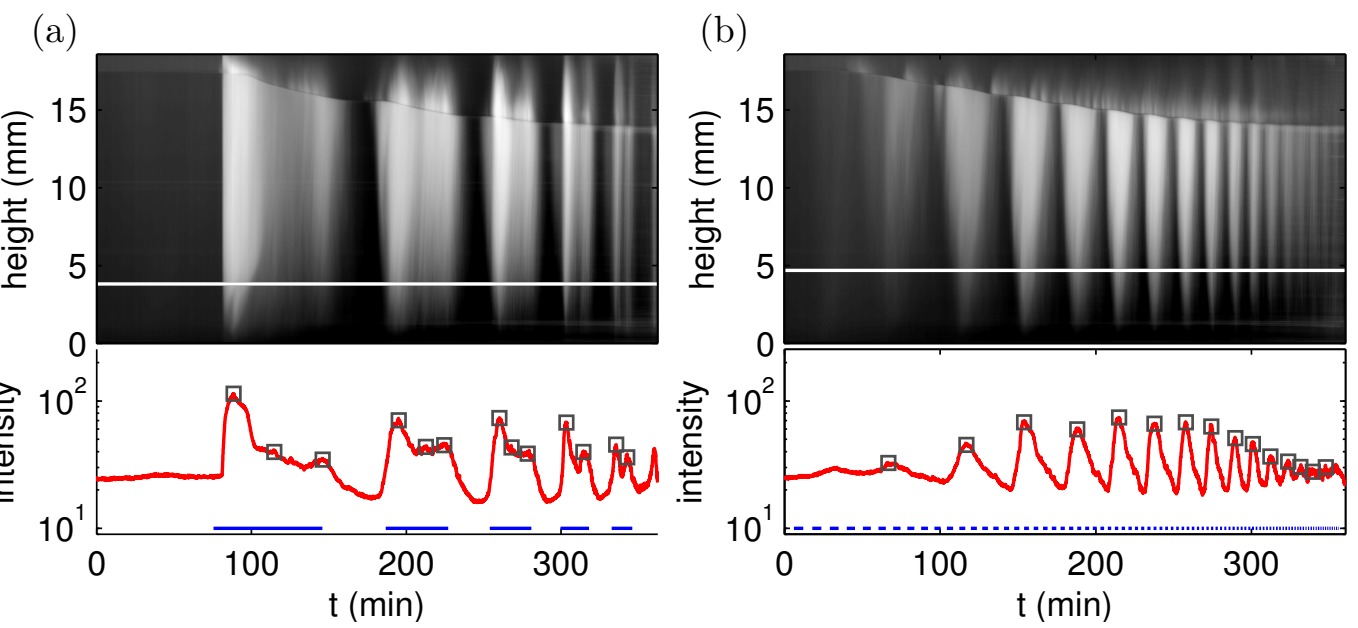

Figure 6.7: Space-time plot and corresponding oscillations for a measurement of (a) $A=0.5$ and $p=1 / 3$ and (b) $A=0.5$ and $p=5$, respectively. The grey squares correspond to the detected peaks in the turbidity, evaluated along the white line indicated in the space-time plot. The interrupted blue line in the bottom indicates the times where the ramp rate takes the value $\xi_{\max }$. 



\section{Discussion}

In the present chapter I revisit the examples of continuously driven phase separation mentioned in chapter 1 of the present thesis in the light of the insights obtained in the subsequent chapters. Section 7.1 is concerned with the implications of the theory for ripening with overall volume growth (section 4.2) for the synthesis of monodisperse colloids and nano-particles. In section 7.2 rain formation is discussed by estimating the driving strength in clouds and applying the model for the time scale developed in chapter 5. Finally geysers are discussed as an instance of episodic precipitation.

\subsection{Synthesis of monodisperse colloids and nano-particles}

Fundamental work on the synthesis of monodisperse colloids goes back to LaMer and Dinegar (1950) and Reiss (1951). The theoretical understanding of the mechanisms that lead to highly monodisperse colloids and nano-crystals is still a topic of active research (Rempel et al., 2009; Clark et al., 2011; Singh et al., 2012). A common feature is a supply of monomers for the growth of particles. I start by giving an estimate of the value of $k$ to show that the theory developed in section 4.2 can also describe experimentally relevant conditions.

\subsubsection{Estimate values of $k$}

For the synthesis of monodisperse silver particles (used for photographic films) the material flux is well defined, and all material constants required to determine the $k$-values have been documented. For the synthesis of $\mathrm{AgBr}$ and $\mathrm{AgCl}$ particles Sugimoto (1992) and Sugimoto et al. (2000) provide material constants and aggregate numbers that allow us to calculate $k$ based on the increase of the total volume of the aggregates, $\xi \mathcal{V}$, the diffusion coefficient $D$, and the Kelvin length $\sigma$,

$$
k=1+\frac{\xi}{4 \pi D \sigma n}=1+\frac{Q_{0} V_{m}}{4 \pi D \sigma N},
$$

where $N$ is the number of aggregates in the sample volume $\mathcal{V}$, and

$$
\xi=V_{m} Q_{0} / \mathcal{V}
$$




\begin{tabular}{lcccc}
\hline$T\left[{ }^{\circ} \mathrm{C}\right]$ & 40 & 50 & 60 & 70 \\
$D\left[\mathrm{~m}^{2} / \mathrm{s}\right]$ & $9.94 \times 10^{-10}$ & $1.26 \times 10^{-9}$ & $1.56 \times 10^{-9}$ & $1.92 \times 10^{-9}$ \\
$C_{\infty}\left[\frac{\mathrm{mol}}{\mathrm{m}^{3}}\right]$ & $1.01 \times 10^{-4}$ & $2.12 \times 10^{-4}$ & $4.34 \times 10^{-4}$ & $8.42 \times 10^{-4}$ \\
$N$ & $3.20 \times 10^{17}$ & $1.25 \times 10^{17}$ & $4.60 \times 10^{16}$ & $2.20 \times 10^{16}$ \\
\hline$k$ & 1.63 & 1.62 & 1.69 & 1.62 \\
\hline
\end{tabular}

Table 7.1: Representative material parameters for the synthesis of monodisperse $\mathrm{AgBr}$ particles (adapted from Sugimoto, 1992) and the corresponding $k$ values as calculated via Eqs. (7.1). The molar volume of $\mathrm{AgBr}$ is $V_{m}=2.9 \times 10^{-5} \mathrm{~m}^{3} / \mathrm{mol}$, and its specific surface energy is $\gamma=1.77 \times 10^{-1} \mathrm{~J} / \mathrm{m}^{2}$. All experiments were conducted with a mass supply rate, $Q_{0}=10^{-3} \mathrm{~mol} / \mathrm{s}$.

\begin{tabular}{lcccc}
\hline$T\left[{ }^{\circ} \mathrm{C}\right]$ & 25 & 30 & 35 & 40 \\
$D\left[\mathrm{~m}^{2} / \mathrm{s}\right]$ & $1.44 \times 10^{-9}$ & $1.64 \times 10^{-9}$ & $1.86 \times 10^{-9}$ & $2.11 \times 10^{-9}$ \\
$C_{\infty}\left[\frac{\mathrm{mol}}{\mathrm{m}^{3}}\right]$ & $5.04 \times 10^{-4}$ & $7.30 \times 10^{-4}$ & $1.04 \times 10^{-3}$ & $1.46 \times 10^{-3}$ \\
$n\left[\mathrm{~m}^{-3}\right]$ & $5.88 \times 10^{13}$ & $5.71 \times 10^{13}$ & $4.24 \times 10^{13}$ & $2.70 \times 10^{13}$ \\
$q_{0}\left[\frac{\mathrm{mol}}{\mathrm{m}^{3} \mathrm{~s}}\right]$ & $5.95 \times 10^{-6}$ & $1.54 \times 10^{-5}$ & $3.86 \times 10^{-5}$ & $8.88 \times 10^{-5}$ \\
\hline$\xi\left[\mathrm{s}^{-1}\right]$ & $1.54 \times 10^{-10}$ & $3.99 \times 10^{-10}$ & $1.00 \times 10^{-9}$ & $2.30 \times 10^{-9}$ \\
$k$ & 6.26 & 9.64 & 19.4 & 43.3 \\
\hline
\end{tabular}

Table 7.2: Material parameters for the synthesis of monodisperse $\mathrm{AgCl}$ particles (adapted from Sugimoto et al., 2000, table 3), and the resulting $k$-values as calculated via Eqs. (7.1). For $\mathrm{AgCl}$ particles the molar volume is $V_{m}=2.59 \times 10^{-5} \mathrm{~m}^{3} / \mathrm{mol}$, and their specific surface energy is $\gamma=1.009 \times 10^{-1} \mathrm{~J} / \mathrm{m}^{2}$.

is provided in terms of the molar volume, $V_{m}$, and the mass supply rate, $Q_{0}$. Finally, the specific surface energy $\gamma$, the buffer temperature $T$, the mean-field monomer concentration $C_{\infty}$, and the molar gas constant $R=8.314 \mathrm{~J} /(\mathrm{mol} \mathrm{K})$ provide the Kelvin length as

$$
\sigma=\frac{2 \gamma V_{m}^{2} C_{\infty}}{R T} .
$$

Table 7.1 provides the resulting $k$-values for different representative sets of $T, D, C_{\infty}$ and $N$ used for the synthesis of $\mathrm{AgBr}$ particles, and table 7.2 provides the $k$ values for the synthesis of $\mathrm{AgCl}$ particles. Also in the latter case the $k$ values are obtained from eq. (7.1), except that Sugimoto et al. (2000) provided the molar injection rate $q_{0}=Q_{0} / \mathcal{V}$ and the number density of droplets, $n=N / \mathcal{V}$. The data shows that the $k$ values selected for the synthesis of monodisperse silver particles lie at $k \simeq 1.6$ for 


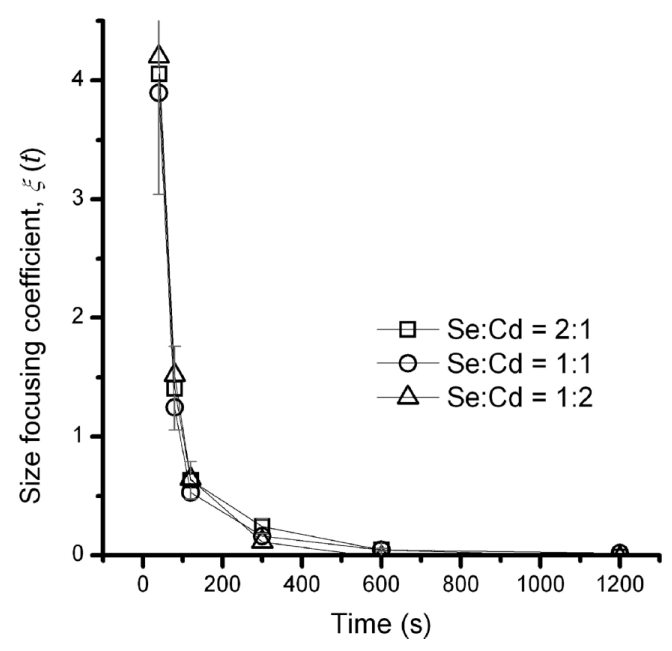

Figure 7.1: Evolution of the size focusing coefficient, that correspond to $k-1$ in the present notation. The observed decay was attributed to a decreasing precursor reaction rate for the production of monomers. The symbols denote ratios of different precursor concentrations. Reprinted from Clark et al. (2011).

$\mathrm{AgBr}-$ particles and in a range between 6 and 43 for $\mathrm{AgCl}$. This choice was obtained by tuning the temperature and the rates $Q_{0}$ or $q_{0}$ for optimal monodispersity of the product. In all cases this resulted in $k$ values larger than $3 / 2$ such that one can profit from the size focusing arising for $k>3 / 2$. In principle, the values of $k$ should be chosen as large as possible to achieve the smallest standard deviation, equation (4.32), and minimise the time required for the synthesis, equation (4.8). In practice, it becomes harder to realise stable and reproducible experimental conditions for large values of $k$, and the heat released in the growth might severely alter the present theory for large growth rates. Follow-up work will have to explore these effects.

For the synthesis of CdSe nanocrystal Clark et al. (2011) extracted the a size focussing coefficient from experiments published by Chan et al. (2010). In fig. 7.1 I reproduce the data for the size focussing coefficient $\xi$ that corresponds to a dimensionless form of our ramp rate $\xi$. In our notation it is equal to $k-1=\xi /(4 \pi D \sigma n)$. The corresponding $k$ values start at $k \approx 5$ at early times, $t \simeq 50 \mathrm{~s}$, and decay to 1 at times, $t \gtrsim 600 \mathrm{~s}$. This is a consequence of a decreasing reaction rate of the precursor reaction that produces the monomers for nano-particle growth. Here I expect, that feeding of the precursor reaction by external addition of material will lead to a constant value of $k$. This in turn enhances size focussing and should lead to narrower distribution. 


\subsubsection{Shape of the distributions and size focussing}

Size focussing in particle synthesis and aggregate growth is nowadays an active field of research and therefore there exist a wealth of literature. Nevertheless, the shape of a distribution undergoing size distribution has never been analysed to my knowledge. Only the first two moments of the distribution are in the focus. In chapter 4 I predict that the asymptotic shape of the distribution depends on the shape of the initial distribution. For values of $k \gtrsim 5$, that are found in experimental realizations, the distribution for all times can be scaled on top of the initial distribution. As a consequence a bimodal distribution stays bimodal for all times, see figs. 4.1 and 4.2. The two peaks move together due to size focussing, thus producing two narrow peaks very close to each other. Especially this finding might be of interest to the community.

\subsection{Rain Formation}

Rain emerges when the air masses in a cloud rise due to topographic constraints, or by encountering a cold front (Mason, 1971; Rogers and Yau, 1989; Pruppacher and Klett, 1997). The drop of pressure in response to the rising of height leads to adiabatic cooling of the air. This in turn changes the solubility of water in the air. Similarly to the phase separation in the experiment on binary fluid demixing this induces a continuous growth of cloud droplets until they reach a size where collisions due to gravity and inertia speed up their growth and trigger rain formation (Bodenschatz et al., 2010). Clement (2008) discussed the micro-physics of the droplet growth, emphasising the importance of the heat of condensation and the impact of solutes in the droplets. Here I augment his study by an estimate of the possible impact of the continuous growth of the droplet volume fraction. I start with estimating the ramp rate $\xi$ for cloud droplet growth and the corresponding values of $k$.

\subsubsection{Growth of cloud droplets: estimating the value of $k$}

Consider a small cloud parcel of volume $V$ that is large as compared to droplet diameters, but sufficiently small that it may be characterized by a fixed composition and temperature $T$. Water droplets constitute a fraction, $v_{d}$, of the volume, and there is also water available in the form of water vapour in the continuous, air-phase of the cloud.

The ideal gas law states that a molar density, $n_{w}$, of the water vapour in air gives rise to a partial pressure $p_{w} V=n_{w} R T$, where $R=8.314 \mathrm{~J} /(\mathrm{mol} \mathrm{K})$ is the molar gas constant. In the presence of water droplets, $p_{w}$, amounts to the saturation vapour 
pressure (Bolton, 1980),

$$
p_{w}^{s}=6.112 \times 10^{-3} \text { bar } \exp \left(\frac{17.67 T}{T+243.5^{\circ} \mathrm{C}}\right),
$$

where $T$ should be inserted in units of ${ }^{\circ} \mathrm{C}$. This equation characterizes the phase diagram of the water-air binary mixture. Henceforth, $p_{w}$ and $n_{w}$ denote the pressure and composition at saturation, and we suppress the superscript $s$.

The molar mass of water, $M_{w}=18 \mathrm{~g} / \mathrm{mol}$, relates the mass, $m_{w}$, of water vapour in the continuous, air-phase of the cloud to the molar density, $m_{w}=M_{w} n_{w}$, such that

$$
m_{w}=M_{w} n_{w}=M_{w} \frac{p_{w} V}{R T} .
$$

Mass conservation relates the changes of the amount of water vapour in the gas phase and the volume fraction of droplets via

$$
\mathrm{d} v_{d}=-\mathrm{d} \frac{m_{w}}{\rho_{w} V}=-\mathrm{d}\left(\frac{M_{w}}{\rho_{w}} \frac{p_{w}}{R T}\right)
$$

where $\rho_{w}=10^{3} \mathrm{~kg} / \mathrm{m}^{3}$, is the density of water in the liquid phase. This equation relates changes of the volume fraction of the water droplet in a cloud, $v_{d}$, to changes of the temperature, $T$, and the saturation pressure of water $p_{w}$. In clouds the temperature changes by adiabatic cooling, when the volume rises in height, $z$, and temperature induced changes of the mass density, $\rho_{w}$, are small. Consequently,

$$
\xi=\dot{v}=-\frac{\mathrm{d} v}{\mathrm{~d} T} \frac{\mathrm{d} T}{\mathrm{~d} t} \simeq-v\left(\frac{1}{p_{w}} \frac{\mathrm{d} p_{w}}{\mathrm{~d} T}-\frac{1}{T}\right) \frac{\mathrm{d} T}{\mathrm{~d} z} \frac{\mathrm{d} z}{\mathrm{~d} t},
$$

where the adiabatic lapse rate, $-\mathrm{d} T / \mathrm{d} z$, takes values of the order of $10^{-2} \mathrm{~K} / \mathrm{m}$ (Wallace and Hobbs, 2006). In fig. 7.2(a) I provide the resulting values of $\xi$ for an upwind wind speed of $\dot{z}=1 \mathrm{~m} / \mathrm{s}$.

To estimate the according $k$ values I first calculate the Kelvin length $\sigma$, eq. (1.4). With the values (Mason, 1971, p. 614, for $10^{\circ} \mathrm{C}$ ) of the interfacial tension of the water-air interface, $\gamma=7.4 \times 10^{-2} \mathrm{~N} / \mathrm{m}$ a molar volume $V_{m}=18 \times 10^{-6} \mathrm{~m}^{3} / \mathrm{mol}$, and an equilibrium volume fraction $\Phi_{e}=10^{-5}$ we get $\sigma=10^{-14} \mathrm{~m}$. The values of $k$ corresponding to the calculated ramp rates, $\xi$, are given in fig. 7.2(b) for a number density of $n=10^{8} \mathrm{~m}^{-3}$ estimated by Ditas et al. (2012) and a diffusion coefficient $D=2.4 \times 10^{-5} \mathrm{~m}^{2} / \mathrm{s}$ (Mason, 1971, p. 614). In warm clouds, where no ice must be formed, $T$ may be expected to take values above $-10^{\circ} \mathrm{C}$. In this temperature range the curve for $k(T)$ does not change noticeably when accounting for the temperature dependence of the material constants such that $k \gtrsim 8$. 

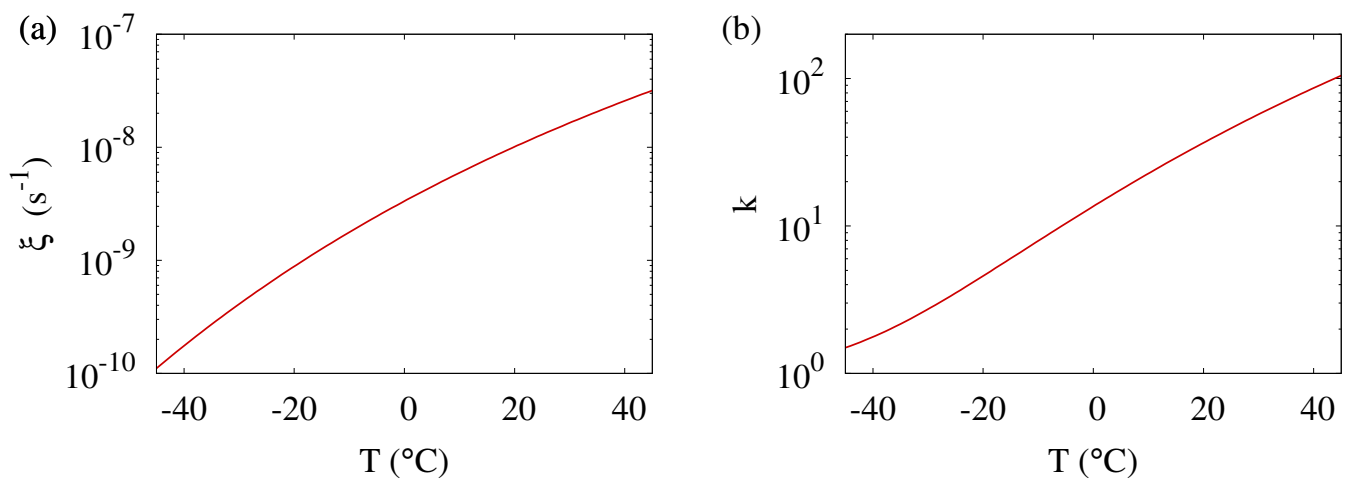

Figure 7.2: Ramp rate for warm clouds. (a) The ramp rate, $\xi$, as a function of the ambient temperature $T$ for a lapse rate $-\mathrm{d} T / \mathrm{d} z=10^{-2} \mathrm{~K} / \mathrm{m}$ and upwind speed of $\dot{z}=1 \mathrm{~m} / \mathrm{s}$. According to eq. (7.5) the ramp rate $\xi$ is proportional to these numbers such that ramp rates for other weather conditions can be estimates by adapting the lapse rate and the upwind speed. (b) The corresponding values of $k=1+\xi /(4 \pi D \sigma n)$.

\subsubsection{Estimates of $\Delta t$ and the bottleneck radius}

To estimate the time scale $\Delta t$ for common situations in warm rain (Beard and Ochs, 1993; Moran and Morgan, 1997) I evaluate the prediction of $\Delta t$, eq. (5.15b). The material constants entering the settling velocity of the droplets are the density contrast of water and air, $\Delta \rho \approx 10^{3} \mathrm{~kg} / \mathrm{m}^{3}$, and the dynamic viscosity of air, $\mu=$ $1.8 \times 10^{-5} \mathrm{~kg} \mathrm{~m}^{-1} \mathrm{~s}^{-1}$ at $10^{\circ} \mathrm{C}$ (Rogers and Yau, 1989, p. 103). Given that the dynamic viscosity of air is much smaller than that of water, this provides a value $\kappa=1.2 \times 10^{8} \mathrm{~m}^{-1} \mathrm{~s}^{-1}$ [cf. eq. (4.33)]. For ramp rates $\xi=2 \times 10^{-9} \cdots 2 \times 10^{-8} \mathrm{~s}^{-1}$, a number density $n=10^{8} \mathrm{~m}^{-3}$ and a collection efficiency of $\varepsilon=0.3$ we then find time scales $\Delta t$, eq. (5.15b), in the range of $10 \mathrm{~min}$ to $40 \mathrm{~min}$, and bottleneck radii, eq. (5.9), between $17 \mu \mathrm{m}$ and $24 \mu \mathrm{m}$.

The values for the time scale $\Delta t$ agree with typical life times of clouds (Beard and Ochs, 1993; McGraw and Liu, 2003) and also the predictions for the bottleneck radius matches expectation (Shaw, 2003; Clement, 2008), see also fig. 1.1. This agreement is remarkable because common estimates (Houghton, 1959; Falkovich et al., 2002; Clement, 2008) based on diffusive ripening processes and growth by collection tend to provide estimates that are rather too large.

\subsubsection{Periodic driving}

The process of cloud formation is much more complex, than a simple up draft of humid air parcels, especially because convection is involved (Stevens, 2005). To estimate the time scales of convection I consider a $1 \mathrm{~km}$ high cloud with wind speed 
of 1 to $10 \mathrm{~m} / \mathrm{s}$. Neglecting asymmetries in the up and down winds we arrive at times of 3 to 30 minutes for one turn over. This reaches the time of the precipitation cycle for small wind speeds. Here the phase locking observed in chapter 6 might become important. In fig. 6.4 precipitation (peak in the turbidity) emerges at the end of the period with a large value of the driving. This would suggest that rain is produced in the top part of the cloud. However, in view of the complexity of clouds the predictions might better be first tested by comparison to model systems for clouds like moist Rayleigh-Bénard-convection (Weidauer et al., 2010), or like experiments in the group of Eberhard Bodenschatz of turbulent Rayleigh-Bénard-convection in a two phase binary gas mixture (Winkel, 2014).

Future work should extent our model also to the case of shrinking droplets, because down welling air parcels correspond to a negative driving, where droplets might dissolve.

\subsection{Geysers and lake eruptions}

Here I briefly describe the mechanism of a $\mathrm{CO}_{2}$ cold-water geyser (Han et al., 2013) to highlight the similarities in the growth of small bubbles with the present modelling of episodic precipitation. Subsequently, I point at another mechanism and explain, how gravity acts as a feedback on the droplet growth rate, that leads to a runaway resulting in the eruption of the geyser.

The basic ingredients of a cold-water geyser are a long vertical channel or conduit that is connected to atmospheric pressure at the top, and a source of $\mathrm{CO}_{2}$-rich water at the bottom of the well. The solubility of $\mathrm{CO}_{2}$ depends on hydrostatic pressure and thus on the vertical position in the channel. As the $\mathrm{CO}_{2}$-rich water fills the channel from below, there is a certain height, where the water becomes supersaturated and bubbles of $\mathrm{CO}_{2}$ nucleate. These tiny bubbles grow in the presence of a source of $\mathrm{CO}_{2}$ by collecting supersaturation, as described for the growth of Brownian particles (section 5.2.1.1). Here the growth does not differ qualitatively from that of cloud droplets or monodisperse colloids.

When the bubbles are affected by gravity, they begin to rise. Like the cloud droplets they can collect other bubbles and enhance their growth, but this mechanism is of minor importance. Gas bubbles are compressible in contrast to liquid droplets, and therefore their volume can grow by expansion due to decreasing hydrostatic pressure on their way to the top of the channel. When the bubbles fill a considerable fraction of the channel, the hydrostatic pressure at the bottom of the channel is decreased. Also the solubility of $\mathrm{CO}_{2}$ decreases and more and more bubbles are generated, which eventually leads to an eruption of the geyser. During the eruption almost all bubbles are removed from the channel and the water level has decreased. Subsequently, the channel is recharged with $\mathrm{CO}_{2}$-rich ground water. A new cycle can start. 
Similar to $\mathrm{CO}_{2}$ geyser some lakes also have a source of $\mathrm{CO}_{2}$ at the bottom. This leads to $\mathrm{CO}_{2}$ saturated or even supersaturated water. Zhang and Kling (2006) and Woods (2010) discussed the trigger of gas release in an eruption or a resorption of the $\mathrm{CO}_{2}$ in upper parts of the lake, where the water is not saturated with $\mathrm{CO}_{2}$.

In conclusion, in a geyser small gas bubbles grow by accretion of supersaturation, and for large bubbles there is a runaway growth due to pressure release in the bottom of the well that leads to the eruption of the geyser. The second growth mechanism is different to the runaway growth considered so far. However, I expect that the time scale between eruptions can still be described via the general concept of modelling time scales by two growth mechanisms separated by a bottleneck. A detailed analysis I leave for further investigations.

\subsection{Summary}

The application of the concepts that have been developed in the present thesis to describe continuously driven phase separation provide valuable new insights for a wealth of different applications. Experimental conditions in the synthesis of monodisperse colloidal particles lie above $k=3 / 2$ where I observe size focussing. This opens new ways in controlling the mechanism of size focussing. Applying the model developed in chapter 5 to the formation of warm rain gives reasonable estimates for the time scale until precipitation occurs and for the crossover from diffusive growth to growth by collection. The example of the geyser showed that the general concept of the the crossover between two growth mechanisms is useful also for the understanding of other oscillatory phenomena. 


\section{Conclusion}

The presented research has shed light into and resolved a fair number of problems that we were dealing with when I started my $\mathrm{PhD}$ thesis. However, of course every answer raised at least one other question. Hence, I will summarize the highlights of resolutions, and point at a few of the new questions.

\section{Ripening versus size focussing}

What is the impact of overall volume growth on ripening? How does it change Ostwald ripening with its broad universal size distribution into an evolution of the droplet-size distributions exhibiting size focussing? To study this crossover an efficient numerical algorithm has been established that allows us to integrate an assembly of droplets in the presence of a material source. The algorithm is designed to run on a logarithmic time axis such that droplet growth can easily be followed over six orders of magnitude in volume. This is necessary because of long transients for small $k$ values.

The insights from the numerical work were formulated in terms of an analytic theory for large $k \approx 50 \cdots 100$, and the model was extended by higher order terms to work also for $k \gtrsim 5$. The numerical data agree perfectly with the theory. I observe size focussing for $k>3 / 2$, in accordance with experiments on the synthesis of monodisperse colloids (see section 7.1 and fig. 1.5). Moreover, all systems with an initial $k>1$ are predicted to approach $k>3 / 2$. For $k<3 / 2$ the growth of droplets is governed by an unstable fixed point at the average radius, like in Ostwald ripening. At $k=3 / 2$ an exchange of stability bifurcation leads to a stable fixed point at the average droplet radius, see fig. 4.12. This stable fixed point is the cause of size focussing observed for $k>3 / 2$.

The resulting shape of the distribution of an assembly of aggregates for $k \gtrsim 5$ is determined by the initial distribution, rather than approaching a universal shape as described by LSW-theory. In fig. 4.1 this was demonstrated for three vastly different types of initial distributions.

Future work will address a theoretical description of $k \lesssim 1$, i.e. the case where droplets evaporate. Moreover, experiments should check the evolution of bimodal distributions. 
(a)

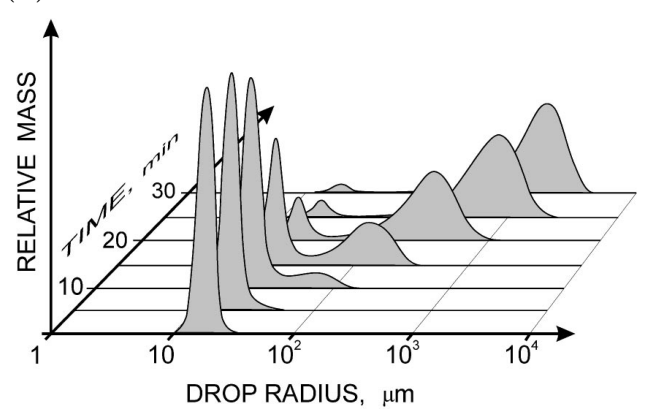

(b)

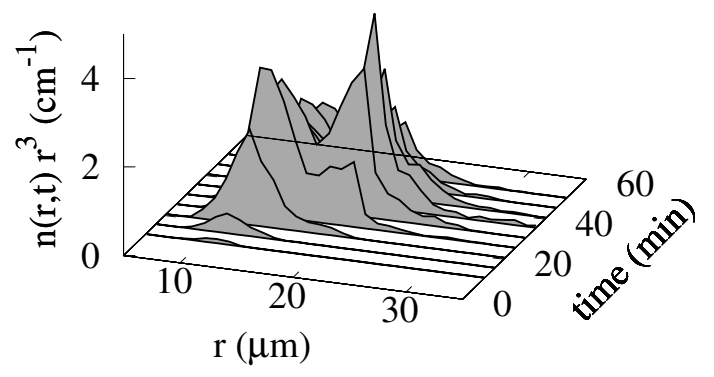

Figure 8.1: (a) Illustration of the evolution of the size distribution in a cloud during the onset of the collision-coalescence process. Reprinted from Shaw (2003). (b) Emerging of bimodal distributions in phase separation of an $\mathrm{IBE}+\mathrm{W}$ mixture at the crossover where sedimentation becomes dominant. The volume fraction per radius (that is proportional to the mass in a given radius bin) is shown as a function of time. This data set was already shown in fig. 4.10 with different axis. Here it is replotted to highlight the similarity of our data to the schematic plot in panel (a) that addresses rain formation.

\section{Brownian particles versus sedimentation}

Ripening and size focussing address the growth of droplets, as long as they can be considered Brownian particles. How does buoyancy effect droplet growth as they become larger? How is the shape of the droplet-size distribution affected by sedimentation? Size distributions in binary fluids had already been measured by Rohloff (2011) and Lapp (2011). However, the influence of sedimentation on the shape of the distribution remained unclear. Careful measurements, where the driving was turned off during sedimentation, reveal bimodal distributions. The peak at larger radii describes the evolution of the sedimenting droplets, growing by collection of small droplets. This is in good agreement with an analytic solution of the continuity equation of the droplet number density, where sedimentation is accounted for by a loss term. Hence, I have established first experimental evidence in well-controlled lab experiments fig. 8.1 that bimodal size distributions emerge in phase separating binary fluids, as suggested also for the evolution of cloud droplets.

The setup for the determination of the size distribution was designed to investigate the droplet sizes around and above the crossover. Further studies should address the predictions for droplet growth well below the crossover size. This can be done by using mass-density matched fluids to reduce the parameter $\kappa$ and therefore shift the bottleneck radius to higher radii, or with a new setup that allows the detection of much smaller droplets. This will possibly also shine light on the mechanism producing new droplets in each oscillation, that remains still uncertain. 


\section{From sedimentation to episodic precipitation}

Sedimentation has been identified as the dominant growth contribution of large droplets. How does sedimentation lead to episodic precipitation? What sets the time scale? Integrating the growth rate of sedimenting droplets leads to a finite time runaway, i.e. the droplet volume diverges at finite times, see section 5.2.1.3 and section 1.5. This provides a mechanism to remove almost all droplet volume and reset the system, such that a new precipitation cycle can start.

The time scale of episodic precipitation is found to be set by the time to reach the crossover from Brownian particle growth to growth by sedimentation. The $k$ values found for the reported measurements of continuously driven phase separation are of the order of $10^{5}$. This suggests to apply the theory of large $k$ values for the growth of Brownian particles. This new perspective allowed me to establish a model of the precipitation cycle, where the oscillation period is in quantitative agreement with the experimental data. The only free parameter in the model is the collection efficiency, that is known from literature by the order of magnitude or from indirect measurements, see section 4.3.4. For turbidity measurements, where the number density of droplets is unknown, there is one fit parameter. However, the unknown droplet number density enters the prediction only with a power of $1 / 7$, such that its uncertainty is strongly suppressed.

The model describes the dependence of the oscillation period on material parameters. Measurements of oscillation periods in a second mixture of binary fluids confirmed the universal description. Hydrodynamic and thermodynamic similarity allows the application of the model to the formation of warm rain. The model provides reasonable values for the time scale and the crossover radius between the diffusive growth and the growth by collisions. Focussing on a right implementation of the driving for cloud models seems more promising to get proper time scales rather than calling for turbulence and inertial effects of the droplets to provide speed-up (Falkovich et al., 2002; Bodenschatz et al., 2010).

\section{Constant versus periodic driving}

For the quantitative test of the prediction of the oscillation period I designed temperature protocols for driving the phase separation that keep the ramp rate as constant as possible. To achieve constant ramp rates I used a scaling representation of the coexistence curve to calculate the temperature ramps. This is done to be consistent with the description of the phase diagram by the theory of critical scaling. On the other hand, applications in clouds exhibit fluctuations or even large scale convection that leads to a periodic variation of the driving. This raises the question how the period of the oscillation is affected by large amplitude periodic perturbations 
of the driving In order to study the periodic driving, I designed temperature protocols for different ratios of the driving period to the undisturbed period. For close to one ratios phase locking of the oscillation is observed whereas for a large ratio only the average driving is important. The system averages over the perturbations and behaves as in the case of constant driving. These experimental observations have been confirmed by applying also a periodic driving to the model of the precipitation cycle established for constant driving. The analytic approximation as well as the numerical solution of the model fit very well to trends observed when varying the period ratio. I expect that these findings will provide a useful input for improved cloud parametrization in climate models (Sant et al., 2013).

In summary, the identification of crossovers and mechanisms that lead to focusing or broadening of size distributions provided a point of view that was very fruitful for the understanding of precipitation in continuously driven phase separation. I am happy that it provided such a wealth of quantitatively new insights. 


\section{Appendix: Material Constants}

Figure 5.1 shows the period, $\Delta t$, of episodic precipitation for different ramp rates, $\xi$. Different data points for a given ramp rate are due to the drift of $\Delta t$ when the pertinent material constants, $D, \sigma$, and $\kappa\left(\Delta \rho, \mu_{b}, \mu_{d}\right)$ change upon moving further away from the critical point. In the following we provide the temperature dependence of these material constants. We cite the data here as they were provided in the original literature (even when we are in doubt that they are accurate to six significant digits for our samples). Upon doing so we denote the mass fraction as $\phi^{m}$ and the molar fraction as $\phi^{n}$, respectively. The resulting temperature dependence of the diffusion coefficient $D$, the Kelvin length $\sigma$ provided by eq. (1.4), and the sedimentation prefactor $\kappa$ provided by eq. (4.33), are summarised in fig. A.1 in order to give easy access to the constants appearing in the predictions eq. (5.15a) and eq. (5.16). The temperature dependence translates to a time dependence when inverting the protocol $T(t)$ of the temperature ramp.

\section{A.1 Isobutoxyethanol and water}

The theoretical curves in figs. 5.7 and 5.8 use data on material parameters from a variety of sources (Steinhoff and Woermann, 1995; Aratono et al., 1990; Doi et al., 2000; Menzel et al., 2003; Douheret et al., 2002). The index $i \in\{\mathrm{IBE}, \mathrm{W}\}$ will be used to refer to material properties of IBE and water, respectively, and in accordance with the phase diagram, fig. 2.2(a), the concentration are always given in terms of $\phi=\phi_{\mathrm{IBE}}$.

\section{A.1.1 Density (based on Doi et al., 2000)}

The densities of the phases are determined by the composition, thermal expansion and molar excess volume,

$$
\rho\left(\phi^{m}, T\right)=\left[\frac{\phi^{m}}{\rho_{\mathrm{IBE}}}+\frac{1-\phi^{m}}{\rho_{\mathrm{W}}}+\left(\frac{\phi^{m}}{M_{\mathrm{IBE}}}+\frac{1-\phi^{m}}{M_{\mathrm{W}}}\right) V_{\mathrm{E}}^{n}\right]^{-1},
$$

where $\rho_{i}=\rho_{i}(T)$ are the (temperature-dependent) densities of the pure substances, $M_{i}$ their molar masses, and $V_{\mathrm{E}}^{n}=V_{\mathrm{E}}^{n}\left(\phi^{n}\right)$ is the molar excess volume. 

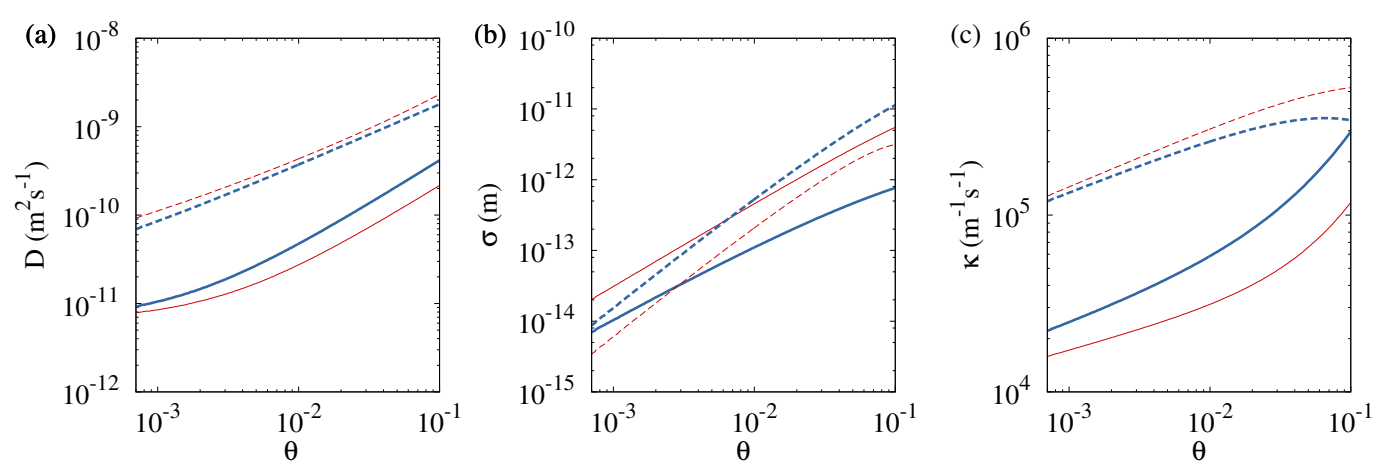

Figure A.1: Material constants. (a) The diffusion coefficient $D$, (b) the Kelvin length $\sigma$, and (c) the sedimentation prefactor $\kappa$ as a function of the reduced temperature $\theta$ for $\mathrm{IBE}+\mathrm{W}$ (solid lines) and $\mathrm{M}+\mathrm{H}$ (dashed lines). The thick blue and the thin red lines show the dependence in the lower and the upper layer of the fluid mixtures, respectively.

\begin{tabular}{lcc} 
& $\rho_{i}\left(T_{0}\right)\left[\mathrm{g} \mathrm{cm}^{-3}\right]$ & $\alpha_{i}\left[\mathrm{~g} \mathrm{~cm}^{-3} \mathrm{~K}^{-1}\right]$ \\
\hline water & 0.997043 & $0.2571 \times 10^{-3}$ \\
IBE & 0.886255 & $0.968 \times 10^{-3}$ \\
\hline
\end{tabular}

Table A.1: Densities and thermal expansion coefficients for water and IBE according to Doi et al. (2000).

The molar masses, $M_{i}$ are $18.01528 \mathrm{~g} / \mathrm{mol}$ for water (PubChem, 2013d) and $118.17416 \mathrm{~g} / \mathrm{mol}$ for IBE (PubChem, 2013a), respectively.

The temperature dependence of the density, $\rho_{i}(T)$, of the pure substances is linearly approximated around $T_{0}=25^{\circ} \mathrm{C}$,

$$
\rho_{i}(T)=\rho_{i}\left(T_{0}\right)-\alpha_{i}\left(T-T_{0}\right)
$$

with fit parameters for $\rho_{i}$ and $\alpha_{i}$ given in table A.1. 


\begin{tabular}{lllll} 
& $A\left[\mathrm{~kg} \mathrm{~m}^{-1} \mathrm{~s}^{-1}\right]$ & $B$ & $C\left[\left({ }^{\circ} \mathrm{C}\right)^{-1}\right]$ & $D\left[{ }^{\circ} \mathrm{C}\right]$ \\
\hline water & $1.002 \times 10^{-3}$ & 1.3272 & 0.001053 & 105 \\
IBE & $3.4 \times 10^{-3}$ & 1.7 & 0.001 & 110 \\
\hline
\end{tabular}

Table A.2: Data of Weast (1988-1989) for the fit coefficients for the viscosity of water and IBE, defined by eq. (A.4). In both cases $T_{0}=20^{\circ} \mathrm{C}$ is used as reference temperature.

Moreover, the molar excess volume is fitted according to Doi et al. (2000):

$$
\begin{aligned}
V_{\mathrm{E}}^{n}\left(\phi^{n}\right) & =\frac{\phi^{n}\left(1-\phi^{n}\right)}{1-G \phi}\left(A_{1}+A_{2} \phi+A_{3} \phi^{2}\right) \\
\text { with } \phi & =1-2 \phi^{n} \\
\text { and } G & =0.975 \\
A_{1} & =-3.079 \mathrm{~cm}^{3} / \mathrm{mol}, \\
A_{2} & =1.801 \mathrm{~cm}^{3} / \mathrm{mol}, \\
A_{3} & =0.839 \mathrm{~cm}^{3} / \mathrm{mol} .
\end{aligned}
$$

A slight temperature dependence of these fit parameters was reported in Doi et al. (2000). However, it is so small that we need not take it into account here.

To get the dependence of the density difference on the reduced temperature the dependence $\phi(\theta)$ (coexistence curve) into eq. (A.1).

\section{A.1.2 Viscosity (own measurements augmented by data of Weast, 1988-1989; Menzel et al., 2003)}

We first provide the data of the pure phases, and then obtain the viscosity of the mixture by appropriate interpolation.

Following Weast (1988-1989) we describe the temperature dependence of the pure substances by

$$
\mu_{i}(T)=A_{i} 10^{\frac{B_{i}\left(T_{0}-T\right)-C_{i}\left(T_{0}-T\right)^{2}}{T+D_{i}}} .
$$

In table A.2 we provide the values for pure water provided in Weast (1988-1989), and parameters of a fit for IBE whose viscosity we determined with an Ubbelohde viscometer type 537 10/I made by Schott. To interpolate the viscosities for a mixed phase of given mass fraction $\phi^{m}$ we use the composition-dependent viscosities at the reference temperature $T_{r}=25^{\circ} \mathrm{C}$ for a homogeneous mixture in the single-phase 
regime (Menzel et al., 2003). The data is fitted with a fifth order polynomial

$$
\begin{aligned}
\mu\left(\phi^{m}, T_{r}\right)=- & 40.66\left(\phi^{m}\right)^{5}+103.44\left(\phi^{m}\right)^{4}-100.32\left(\phi^{m}\right)^{3} \\
& +39.35\left(\phi^{m}\right)^{2}+0.17 \phi^{m}+0.91 \\
= & w_{\mu}\left(\phi^{m}\right) \mu_{\mathrm{IBE}}\left(T=25^{\circ} \mathrm{C}\right)+\left[1-w_{\mu}\left(\phi^{m}\right)\right] \mu_{\mathrm{W}}\left(T=25^{\circ} \mathrm{C}\right),
\end{aligned}
$$

where the latter equation defines the dimensionless, non-linear weight function $w_{\mu}$ that expresses $\mu\left(\phi^{m}, T=25^{\circ} \mathrm{C}\right)$ as a function of the viscosities of the pure substances $\mu_{\mathrm{IBE}}\left(T=25^{\circ} \mathrm{C}\right)$ and $\mu_{\mathrm{W}}\left(T=25^{\circ} \mathrm{C}\right)$, respectively.

Assuming that this weight function is not varying substantially in the temperature range of our measurements, we can use the interpolation eq. (A.5b) to determine the viscosity of the mixture also at other temperatures. After all, the temperature dependence $\mu_{\mathrm{IBE}}(T)$ and $\mu_{\mathrm{W}}(T)$ were provided by eq. (A.4) with coefficients in table A.2. To check the strong assumption entering this interpolation,

$$
\mu\left(\phi^{m}, T\right)=w_{\mu}\left(\phi^{m}\right) \mu_{\mathrm{IBE}}(T)+\left[1-w_{\mu}\left(\phi^{m}\right)\right] \mu_{\mathrm{W}}(T),
$$

we measured the viscosity of the two coexisting phases at $T=40^{\circ} \mathrm{C}$. For both phases the prediction of eq. (A.6) was accurate to within $2 \%$. This is sufficient for our purposes.

\section{A.1.3 Diffusion coefficient (based on Steinhoff and Woermann, 1995)}

The renormalisation group theory predicts that the diffusion coefficient vanishes when the critical point is approached. On the other hand, the renormalisation group theory is precise only in the vicinity of the critical point, and its application to interdiffusion coefficients has been a source of controversy (Sengers, 1985; Das et al., 2006). For this reason we choose to rely upon interpolations of experimental data. We do not expect that our data follow the critical exponents because the temperatures in our experiments lie outside the critical region. Hence, we fitted the data of Steinhoff and Woermann (1995) with the following expression:

$$
D_{i}(\theta)=D_{\mathrm{c}}+\delta_{i} \theta
$$

with $D_{\mathrm{c}}=6.4 \times 10^{-12} \mathrm{~m}^{2} / \mathrm{s}, \delta_{\mathrm{IBE}}=2.1 \times 10^{-9} \mathrm{~m}^{2} / \mathrm{s}$ and $\delta_{\mathrm{W}}=4.1 \times 10^{-9} \mathrm{~m}^{2} / \mathrm{s}$.

\section{A.1.4 Interfacial tension (based on Aratono et al., 1990)}

The interfacial tension vanishes at the critical temperature, and its dependence at higher temperatures can be represented by a power law

$$
\gamma(\theta)=\gamma_{0} \theta^{\alpha_{\gamma}}
$$




\begin{tabular}{llll} 
& $a_{0}\left[\mathrm{~g} \mathrm{~cm}^{-3}\right]$ & $a_{1}\left[\mathrm{~g} \mathrm{~cm}^{-3} \mathrm{~K}^{-1}\right]$ & $a_{2}\left[\mathrm{~g} \mathrm{~cm}^{-3} \mathrm{~K}^{-2}\right]$ \\
\hline methanol & 1.382 & $-3.135 \times 10^{-3}$ & $3.813 \times 10^{-6}$ \\
hexane & 0.6839 & $6.989 \times 10^{-4}$ & $-2.656 \times 10^{-6}$ \\
\hline
\end{tabular}

Table A.3: Coefficients of the density (Abbas et al., 1997).

where a fit to the data of Aratono et al. (1990) yields $\gamma_{0}=7.3 \times 10^{-4} \mathrm{~N} / \mathrm{m}$ and $\alpha_{\gamma}=1.2$.

\section{A.1.5 Molar volume (based on Douheret et al., 2002)}

According to Douheret et al. (2002) the molar volume $V^{n}$ can be approximated by

$$
V^{n}=\phi^{n} V_{\mathrm{IBE}}^{n}+\left(1-\phi^{n}\right) V_{\mathrm{W}}^{n}
$$

with $V_{\mathrm{IBE}}^{n}=124 \mathrm{~cm}^{3} / \mathrm{mol}$ and $V_{\mathrm{W}}^{n}=15.98 \mathrm{~cm}^{3} / \mathrm{mol}$.

\section{A.2 Methanol and hexane}

In this subsection the index $i \in\{M, H\}$ denotes material constants of the methanol and hexane, respectively, and concentrations refer to methanol, $\phi=\phi_{\mathrm{M}}$.

\section{A.2.1 Density (based on Abbas et al., 1997; Orge et al., 1997)}

The densities are again calculated according to eq. (A.1). In this case the molar mass is $32.04186 \mathrm{~g} / \mathrm{mol}$ for methanol (PubChem, 2013c) and $86.17536 \mathrm{~g} / \mathrm{mol}$ for hexane (PubChem, 2013b). The temperature dependence of the pure substances amounts to (Abbas et al., 1997)

$$
\rho_{i}(T)=a_{0}+a_{1} T+a_{2} T^{2}
$$

with coefficients given in table A.3. The excess volume is expressed as (Orge et al., 1997)

$$
\begin{aligned}
V_{E}^{n}\left(\phi^{n}\right) & =\phi^{n}\left(1-\phi^{n}\right)\left[B_{0}+B_{1} \phi+B_{2} \phi^{2}\right] \\
\text { with } \phi & =1-2 \phi^{n} \\
\text { and } \quad B_{0} & =2.0741 \mathrm{~cm}^{3} / \mathrm{mol}, \\
B_{1} & =0.3195 \mathrm{~cm}^{3} / \mathrm{mol}, \\
B_{2} & =1.7733 \mathrm{~cm}^{3} / \mathrm{mol} .
\end{aligned}
$$




\section{A.2.2 Viscosity (based on Assael and Polimatidou, 1994; Eicher and Zwolinski, 1972; Orge et al., 1997)}

We first provide the data of the pure phases, and then obtain the viscosity of the mixture by appropriate interpolation.

The viscosity of pure methanol (Assael and Polimatidou, 1994) is

$$
\mu_{\mathrm{M}}(T)=A \exp (B / T)
$$

with $A=8.203 \times 10^{-6} \mathrm{~Pa} \mathrm{~s}$ and $B=1251.4 \mathrm{~K}$.

For hexane our analysis is based on the kinematic viscosity $\nu_{\mathrm{H}}$ provided in Eicher and Zwolinski (1972)

$$
\nu_{\mathrm{H}}(T)=\nu^{\prime}\left(\frac{T}{T^{\prime}}\right)^{n} \exp \left(\frac{B\left(T^{\prime}-T\right)}{\left(T^{\prime}-T_{0}\right)\left(T-T_{0}\right)}\right)
$$

with $n=-2.24057, B=4.78496 \mathrm{~K}$ and $T_{0}=222.468 \mathrm{~K}$, reference viscosity $\nu^{\prime}=$ $0.4604 \times 10^{-10} \mathrm{~m}^{2} / \mathrm{s}$, and reference temperature $T^{\prime}=296.267 \mathrm{~K}$. Together with the density of hexane, which is provided in eq. (A.10), this provides the dynamic viscosity $\mu_{\mathrm{H}}=\rho_{\mathrm{H}} \nu_{\mathrm{H}}$.

The viscosity of the mixture is obtained by interpolating based on the excess viscosity provided in Orge et al. (1997)

$$
\begin{aligned}
\mu\left(\phi^{n}, T\right) & =\phi^{n} \mu_{\mathrm{M}}(T)+\left(1-\phi^{n}\right) \mu_{\mathrm{H}}(T)+\phi^{n}\left(1-\phi^{n}\right)\left[B_{0}+B_{1}\left(1-2 \phi^{n}(\mathrm{~A} .14)\right.\right. \\
\text { with } B_{0} & =-1.83 \times 10^{-4} \mathrm{~kg} \mathrm{~m}^{-1} \mathrm{~s}^{-1} \\
B_{1} & =0.91 \times 10^{-4} \mathrm{~kg} \mathrm{~m}^{-1} \mathrm{~s}^{-1}
\end{aligned}
$$

\section{A.2.3 Diffusion coefficient (based on Clark and Rowley, 1986)}

The dependence of the diffusion coefficient $D\left(\phi^{n}, \theta\right)$ on the concentration $\phi^{n}$ of the mixture and on the reduced temperature $\theta$ can be approximated by (Clark and Rowley, 1986)

$$
D\left(\phi^{n}, \theta\right)=A_{0}+A_{1} \phi^{n}+A_{2}\left(\phi^{n}\right)^{2}+A_{3}\left(\phi^{n}\right)^{3}+A_{4}\left(\phi^{n}\right)^{4}+A_{\theta} \theta^{0.68516} \text { A.15) }
$$




$$
\begin{aligned}
\text { with } \quad A_{0} & =3.2457 \times 10^{-9} \mathrm{~m}^{2} / \mathrm{s}, \\
A_{1} & =-1.68497 \times 10^{-8} \mathrm{~m}^{2} / \mathrm{s}, \\
A_{2} & =3.63103 \times 10^{-8} \mathrm{~m}^{2} / \mathrm{s}, \\
A_{3} & =-4.1949 \times 10^{-8} \mathrm{~m}^{2} / \mathrm{s}, \\
A_{4} & =2.223 \times 10^{-8} \mathrm{~m}^{2} / \mathrm{s}, \\
\text { and } \quad A_{\theta} & =2.5067 \times 10^{-9} \mathrm{~m}^{2} / \mathrm{s} .
\end{aligned}
$$

Similarly to the expression eq. (A.7) the fit for the $\mathrm{M}+\mathrm{H}$ mixture involves a constant background contribution, and the singular contribution expected from the theory of critical phenomena. Clark and Rowley (1986) fitted the composition dependence of the background contribution by a forth-order polynomial in $\phi^{n}$, and introduced the term $A_{5} \theta^{0.68516}$ to account for the singular contribution to the diffusion. The latter term vanishes at $T=T_{c}$ with the appropriate critical scaling exponent, 0.68516 .

\section{A.2.4 Interfacial tension (according to Abbas et al., 1997)}

Data of interfacial tension (Abbas et al., 1997) are parametrised according to eq. (A.8) with $\gamma_{0}=3.631 \times 10^{-2} \mathrm{~N} / \mathrm{m}$ and $\alpha_{\gamma}=1.65$. This data lies beyond the critical region of $\theta<10^{-2.5}$ where scaling with a critical exponents is expected (Abbas et al., 1997).

\section{A.2.5 Molar volume (according to Maruyama et al., 1995)}

The molar volume is interpolated with eq. (A.9) with $V^{n}=41.1 \mathrm{~cm}^{3} / \mathrm{mol}$ for methanol and $V^{n}=133.2 \mathrm{~cm}^{3} / \mathrm{mol}$ for hexane (Maruyama et al., 1995). 



\section{Bibliography}

Aarts, D. G. A. L., Dullens, R. P. A., and Lekkerkerker, H. N. W. Interfacial dynamics in demixing systems with ultralow interfacial tension. New Journal of Physics, 7:40, 2005. Cited on pages 2 and 74 .

Abbas, S., Satherley, J., and Penfold, R. The liquid-liquid coexistence curve and the interfacial tension of the methanol-n-hexane system. Journal of the Chemical Society, Faraday Transactions, 93(11):2083-2089, 1997. Cited on pages 17, 119, and 121.

Ahnert, K. and Abel, M. Numerical differentiation of experimental data: local versus global methods. Computer Physics Communications, 177(10):764 - 774, 2007. Cited on page 24.

Aizpiri, A. G., Correa, J. A., Rubio, R. G., and Pena, M. D. Coexistence curve of methanol+n-heptane: Range of simple scaling and critical amplitudes. Physical Review B, 41:9003-9012, 1990. Cited on page 18.

Aratono, M., Nakayama, S., Ikeda, N., and Motomura, K. Thermodynamic consideration on the interface formation of water and ethylene glycol isobutyl ether mixture. Colloid \& Polymer Science, 268(9):877-882, 1990. Cited on pages 115, 118 , and 119.

Assael, M. J. and Polimatidou, S. K. Measurements of the viscosity of alcohols in the temperature range $290-340 \mathrm{~K}$ at pressures up to $30 \mathrm{MPa}$. International Journal of Thermophysics, 15(1):95-107, 1994. Cited on page 120.

Auernhammer, G. K., Vollmer, D., and Vollmer, J. Oscillatory instabilities in phase separation of binary mixtures: Fixing the thermodynamic driving. The Journal of Chemical Physics, 123(13):134511-8, 2005. Cited on pages 6, 18, 19, 21, 22, and 25.

Barenblatt, G. I. Scaling. Cambridge Texts in Applied Mathematics. Cambridge University Press, Cambridge, New York, 2003. Cited on page 8.

Beard, K. V. and Ochs, H. T. Warm-rain initiation: An overview of microphysical mechanisms. Journal of Applied Meteorology, 32:608 - 625, 1993. Cited on pages 66, 80, 84, 85, and 108 .

Becker, R. Theorie der Wärme. Heidelberger Taschenbücher. Springer, 1985. Cited on page 1 .

Benczik, I. J. and Vollmer, J. A reactive-flow model of phase separation in fluid binary mixtures with continuously ramped temperature. EPL (Europhysics Letters), 91 (3):

36 003, 2010. Cited on pages 6 and 74.

Benczik, I. J. and Vollmer, J. A diffusion-induced transition in the phase separation of binary fluid mixtures subjected to a temperature ramp. EPL (Europhysics Letters), 100(1):16001, 2012. Cited on page 74. 
Binder, K. and Stauffer, D. Statistical theory of nucleation, condensation and coagulation. Advances in Physics, 25:343-396, 1976. Cited on page 74.

Bodenschatz, E., Malinowski, S. P., Shaw, R. A., and Stratmann, F. Can we understand clouds without turbulence? Science, 327(5968):970-971, 2010. Cited on pages 106 and 113 .

Bolton, D. The computation of equivalent potential temperature. Monthly Weather Review, 108:1046 - 1053, 1980. Cited on page 107.

Bray, A. Theory of phase-ordering kinetics. Advances in Physics, 43(3):357-459, 1994. Cited on pages 6 and 8.

Cashman, K. V. and Sparks, R. S. J. How volcanoes work: A 25 year perspective. Geological Society of America Bulletin, 125(5-6):664-690, 2013. Cited on page 1.

Cates, M. E., Vollmer, J., Wagner, A., and Vollmer, D. Phase separation in binary fluid mixtures with continuously ramped temperature. Philosophical Transactions of the Royal Society A, 361(1805):793-807, 2003. Cited on pages 19 and 21.

Cau, F. and Lacelle, S. Late-stage phase separation and sedimentation in a binary liquid mixture. Physical Review E, 47:1429-1432, 1993. Cited on page 2.

Chan, E. M., Xu, C., Mao, A. W., Han, G., Owen, J. S., Cohen, B. E., and Milliron, D. J. Reproducible, high-throughput synthesis of colloidal nanocrystals for optimization in multidimensional parameter space. Nano Letters, 10(5):18741885, 2010. Cited on page 105.

Chartrand, R. Numerical differentiation of noisy, nonsmooth data. ISRN Applied Mathematics, 2011:164564, 2011. Cited on pages 24 and 96.

Clark, M. D., Kumar, S. K., Owen, J. S., and Chan, E. M. Focusing nanocrystal size distributions via production control. Nano Letters, 11(5):1976-1980, 2011. Cited on pages $3,9,10,45,48,74,103$, and 105 .

Clark, W. M. and Rowley, R. L. The mutual diffusion coefficient of methanol-nhexane near the consolute point. AIChE Journal, 32(7):1125-1131, 1986. Cited on pages 120 and 121.

Clement, C. F. Mass Transfer to Aerosols, chapter 3, pages 49-89. Blackwell Publishing Ltd., 2008. Cited on pages 106 and 108.

Das, S. K., Fisher, M. E., Sengers, J. V., Horbach, J., and Binder, K. Critical dynamics in a binary fluid: Simulations and finite-size scaling. Physical Review Letters, 97:025702, 2006. Cited on page 118.

de Rooy, W. C., Bechtold, P., Fröhlich, K., Hohenegger, C., Jonker, H., Mironov, D., Pier Siebesma, A., Teixeira, J., and Yano, J.-I. Entrainment and detrainment in cumulus convection: an overview. Quarterly Journal of the Royal Meteorological Society, 139(670):1-19, 2013. Cited on page 1. 
Dean, K. M., Babayco, C. B., Sluss, D. R. B., and Williamson, J. C. The accuracy of liquid-liquid phase transition temperatures determined from semiautomated light scattering measurements. Journal of Chemical Physics, 133:074506, 2010. Cited on page 17 .

Ditas, F., Shaw, R. A., Siebert, H., Simmel, M., Wehner, B., and Wiedensohler, A. Aerosols-cloud microphysics-thermodynamics-turbulence: evaluating supersaturation in a marine stratocumulus cloud. Atmospheric Chemistry and Physics, 12(5): 2459-2468, 2012. Cited on page 107.

Doi, H., Tamura, K., and Murakami, S. Thermodynamic properties of aqueous solution of 2-isobutoxyethanol at $\mathrm{T}=(293.15,298.15$, and 303.15$) \mathrm{K}$, below and above LCST. The Journal of Chemical Thermodynamics, 32(6):729-741, 2000. Cited on pages 115, 116, and 117.

Douheret, G., Davis, M. I., Reis, J. C. R., Fjellanger, I. J., Vaage, M. B., and Hoiland, H. Aggregative processes in aqueous solutions of isomeric 2-butoxyethanols at 298.15 K. Physical Chemistry Chemical Physics, 4(24):6034-6042, 2002. Cited on pages 115 and 119 .

Eicher, L. D. and Zwolinski, B. J. Molecular structure and shear viscosity. Isomeric hexanes. The Journal of Physical Chemistry, 76(22):3295-3300, 1972. Cited on page 120.

Emmanuel, S. and Berkowitz, B. An experimental analogue for convection and phase separation in hydrothermal systems. Journal of Geophysical Research, 111:B09103, 2006. Cited on pages 4 and 17 .

Ernst, M. Evolution of droplet size distributions in episodic precipitation with time-dependent driving. Bachelor's thesis, Georg-August-Universität Göttingen, 2014. Cited on pages 13 and 58.

Falkovich, G., Fouxon, A., and Stepanov, M. G. Acceleration of rain initiation by cloud turbulence. Nature, 419(6903):151-154, 2002. Cited on pages 108 and 113.

Farjoun, Y. and Neu, J. C. Aggregation according to classical kinetics: From nucleation to coarsening. Physical Review E, 83:051607, 2011. Cited on page 74.

Grabowski, W. W. and Wang, L.-P. Growth of cloud droplets in a turbulent environment. Annual Review of Fluid Mechanics, 45(1):293-324, 2013. Cited on page 2 .

Guyon, E., Hulin, J.-P., Petit, L., and Mitescu, C. D. Physical Hydrodynamics. Oxford Univ. Press, Oxford, 2001. translation from French: 'Hydrodynamique Physique', 1991. Cited on page 61.

Han, W. S., Lu, M., McPherson, B. J., Keating, E. H., Moore, J., Park, E., Watson, Z. T., and Jung, N.-H. Characteristics of $\mathrm{CO}_{2}$-driven cold-water geyser, Crystal Geyser in Utah: experimental observation and mechanism analyses. Geofluids, 13 (3):283-297, 2013. Cited on pages 1 and 109.

Houghton, H. G. Cloud physics. Science, 129:307-313, 1959. Cited on pages 2 and 108. 
Hoyt, J. J. Metallic alloys: All particles are equal. Nature Materials, 10:652-654, 2011. Cited on page 43 .

Huang, J. S., Goldburg, W. I., and Bjerkaas, A. W. Study of phase separation in a critical binary liquid mixture: Spinodal decomposition. Physical Review Letters, 32:921-923, 1974. Cited on page 17.

Ingebritsen, S. E. and Rojstaczer, S. A. Controls on geyser periodicity. Science, 262 (5135):889-892, 1993. Cited on page 1.

Jameson, A. R. and Kostinski, A. B. When is rain steady? Journal of Applied Meteorology, 41:83 - 90, 2002. Cited on page 1.

Kalwarczyk, T., Ziebacz, N., Fiałkowski, M., and Hołyst, R. Late stage of the phaseseparation process: Coalescence-induced coalescence, gravitational sedimentation, and collective evaporation mechanisms. Langmuir, 24(13):6433-6440, 2008. Cited on page 2 .

Klein, E. and Moisar, E. Elektronenmikroskopische und nephelometrische Untersuchungen über das Kornwachstum von Silberhalogenidkristallen. Berichte der Bunsengesellschaft für physikalische Chemie, 67(4):349-355, 1963. Cited on page 10 .

Kostinski, A. B. and Shaw, R. A. Fluctuations and luck in droplet growth by coalescence. Bulletin of the American Meteorological Society, 86:235-244, 2005. Cited on pages $2,3,11,12,74$, and 85 .

Kwon, S. G. and Hyeon, T. Formation mechanisms of uniform nanocrystals via hot-injection and heat-up methods. Small, 7(19):2685-2702, 2011. Cited on page 10 .

LaMer, V. K. and Dinegar, R. H. Theory, production and mechanism of formation of monodispersed hydrosols. Journal of the American Chemical Society, 72(11): 4847-4854, 1950. Cited on pages 9 and 103.

Landau, L. D. and Lifshitz, E. M. Lehrbuch der theoretischen Physik, volume X. Physikalische Kinetik. Akademie, Berlin, 1983. Cited on page 8.

Lapp, T. Evolution of Droplet Distributions in Hydrodynamic Systems. PhD thesis, Georg-August-Universität, Göttingen, 2011. Cited on pages 12, 13, 17, 20, 26, 69, and 112 .

Lapp, T., Rohloff, M., Vollmer, J., and Hof, B. Particle tracking for polydisperse sedimenting droplets in phase separation. Experiments in Fluids, 52(5):1187-1200, 2012. Cited on pages 6, 12, 21, and 26.

Lifshitz, I. and Slyozov, V. The kinetics of precipitation from supersaturated solid solutions. Journal of Physics and Chemistry of Solids, 19:35 - 50, 1961. Cited on pages $8,41,75$, and 76 .

Marder, M. Correlations and Ostwald ripening. Physical Review A, 36:858-874, 1987. Cited on page 76 . 
Maruyama, K., Kawase, S., Tamaki, S., and Okazaki, H. Thermodynamic aspects of the Rayleigh and Brillouin scattering from a binary liquid mixture: The hexanemethanol system. The Journal of Physical Chemistry, 99(26):10644-10647, 1995. Cited on page 121.

Mason, B. J. The Physics of Clouds. Oxford University Press, $2^{\text {nd }}$ edition, 1971. Cited on pages 106 and 107.

McGraw, R. and Liu, Y. Kinetic potential and barrier crossing: A model for warm cloud drizzle formation. Physical Review Letters, 90:018501, 2003. Cited on pages 2 and 108.

Menzel, K., Mirzaev, S. Z., and Kaatze, U. Crossover behavior in micellar solutions with lower critical demixing point: Broadband ultrasonic spectrometry of the isobutoxyethanol-water system. Physical Review E, 68(1):011501, 2003. Cited on pages 115,117 , and 118 .

Mirzaev, S. Z., Heimburg, T., and Kaatze, U. Critical behavior of polystyrenecyclohexane: Heat capacity and mass density. Physical Review E, 82:061502, 2010. Cited on page 6.

Moran, J. and Morgan, M. Meteorolgy: the atmosphere and the science of weather. Prentice-Hall, Upper Saddle River NJ, $5^{\text {th }}$ edition, 1997. Cited on page 108.

Nakata, M., Dobashi, T., Kuwahara, N., and Kaneko, M. Coexistence curve and diameter of the system ethylene glycol mono-isobutyl ether + water. Journal of the Chemical Society, Faraday Transactions 2, 78:1801-1810, 1982. Cited on page 19.

Orge, B., Iglesias, M., Rodríguez, A., Canosa, J. M., and Tojo, J. Mixing properties of (methanol, ethanol, or 1-propanol) with (n-pentane, n-hexane, n-heptane and n-octane) at $298.15 \mathrm{k}$. Fluid Phase Equilibria, 133(1-2):213-227, 1997. Cited on pages 119 and 120 .

Ostwald, W. Über die vermeintliche Isomerie des roten und gelben Quecksilberoxyds und die Oberflächenspannung fester Körper. Zeitschrift für Physikalische Chemie, 34:495 - 503, 1900. Cited on page 7.

Pikovsky, A., Rosenblum, M., and Kurths, J. Synchronization: a universal concept in nonlinear sciences. Cambridge University Press, 2003. Cited on page 95.

Pruppacher, H. R. and Klett, J. D. Microphysics of Clouds and Precipitation. Kluwer Academic Publications, $2^{\text {nd }}$ edition, 1997. Cited on page 106.

PubChem, N. Pubchem compound database, 2013a. CID=521158 (17 July 2013). Cited on page 116.

PubChem, N. Pubchem compound database, 2013b. CID=8058 (17 July 2013). Cited on page 119.

PubChem, N. Pubchem compound database, 2013c. CID=887 (17 July 2013). Cited on page 119. 
PubChem, N. Pubchem compound database, 2013d. CID=962 (17 July 2013). Cited on page 116.

Radmilovic, V., Ophus, C., Marquis, E. A., Rossell, M. D., Tolley, A., Gautam, A., Asta, M., and Dahmen, U. Highly monodisperse core-shell particles created by solid-state reactions. Nature Materials, 10:710 -715, 2011. Cited on page 43.

Ratke, L. and Host, M. Convective contributions to Ostwald ripening in dispersions at low Peclet numbers. Journal of Colloid and Interface Science, 141(1):226 - 238, 1991. Cited on page 76 .

Ratke, L. and Voorhees, P. W. Growth and Coarsening - Ostwald Ripening in Material Processing. Engineering Materials. Springer Berlin Heidelberg, 2002. Cited on page 1.

Reiss, H. The growth of uniform colloidal dispersions. The Journal of Chemical Physics, 19(4):482-487, 1951. Cited on pages 10, 43, and 103.

Rempel, J. Y., Bawendi, M. G., and Jensen, K. F. Insights into the kinetics of semiconductor nanocrystal nucleation and growth. Journal of the American Chemical Society, 131(12):4479-4489, 2009. Cited on page 103.

Rogers, R. and Yau, M. A Short Course in Cloud Physics, volume 113 of International Series in natural philosophy. Pergamon Press, Oxford, $3^{\text {rd }}$ edition, 1989. Cited on pages 106 and 108.

Rohloff, M. Measuring the droplet-size and velocity distributions in binary phase separation. Diploma Thesis, Georg-August-Universität Göttingen, 2011. Cited on pages $12,17,20,26,28$, and 112 .

Rohloff, M., Lapp, T., and Vollmer, J. Comment on "a test-tube model for rainfall" by Wilkinson Michael. EPL (Europhysics Letters), 108(3):30005, 2014. Cited on page 13.

Rohloff, M., Lapp, T., and Vollmer, J. Crossing the bottleneck of rain formation. arXiv:1411.7882v1 [cond-mat.stat-mech], 2015. Cited on pages 13, 18, and 70.

Rudin, L. I., Osher, S., and Fatemi, E. Nonlinear total variation based noise removal algorithms. Physica D: Nonlinear Phenomena, 60(1-4):259-268, 1992. Cited on page 24 .

Sam, E. M., Hayase, Y., Auernhammer, G. K., and Vollmer, D. Pattern formation in phase separating binary mixtures. Physical Chemistry Chemical Physics, 13: 13333-13340, 2011. Cited on page 17.

Sant, V., Lohmann, U., and Seifert, A. Performance of a triclass parameterization for the collision-coalescence process in shallow clouds. Journal of the Atmospheric Sciences, 70(6):1744 - 1767, 2013. Cited on page 114.

Sengers, J. V. Transport properties of fluids near critical points. International Journal of Thermophysics, 6:2003-232, 1985. Cited on page 118.

Shaw, R. A. Particle-turbulence interactions in atmospheric clouds. Annual Review of Fluid Mechanics, 35(1):183-227, 2003. Cited on pages 2, 108, and 112. 
Singh, A., Puri, S., and Dasgupta, C. Growth kinetics of nanoclusters in solution. The Journal of Physical Chemistry B, 116(15):4519-4523, 2012. Cited on page 103.

Slezov, V. V. Kinetics of First-Order Phase Transitions. Wiley-VCH, Weinheim, 2009. Cited on pages 50 and 74 .

Slezov, V. and Schmelzer, J. Kinetics of formation and growth of a new phase with a definite stoichiometric composition. Journal of Physics and Chemistry of Solids, $55(3): 243$ - 251, 1994. Cited on page 29.

Snyder, V., Alkemper, J., and Voorhees, P. Transient Ostwald ripening and the disagreement between steady-state coarsening theory and experiment. Acta Materialia, 49(4):699 - 709, 2001. Cited on page 76.

Sowers, K. L., Swartz, B., and Krauss, T. D. Chemical mechanisms of semiconductor nanocrystal synthesis. Chemistry of Materials, 25(8):1351-1362, 2013. Cited on pages 1 and 10.

Stanley, H. Introduction to Phase Transitions and Critical Phenomena. International series of monographs on physics. Oxford University Press, 1971. Cited on pages 1 and 19 .

Steinhoff, B. and Woermann, D. Slowing down of the kinetics of liquid/liquid phase separation along the binodal curve of a binary liquid mixture with a miscibility gap approaching the critical point. The Journal of Chemical Physics, 103(20): 8985-8992, 1995. Cited on pages 115 and 118.

Stevens, B. Atmospheric moist convection. Annual Review of Earth and Planetary Sciences, 33(1):605-643, 2005. Cited on page 108.

Stevens, B. and Feingold, G. Untangling aerosol effects on clouds and precipitation in a buffered system. Nature, 461:607-613, 2009. Cited on page 2.

Stickel, J. J. Data smoothing and numerical differentiation by a regularization method. Computers $\&$ Chemical Engineering, 34(4):467 - 475, 2010. Cited on page 24 .

Sugimoto, T. The theory of the nucleation of monodisperse particles in open systems and its application to AgBr systems. Journal of Colloid and Interface Science, 150 (1):208 - 225, 1992. Cited on pages 45, 103, and 104.

Sugimoto, T., Shiba, F., Sekiguchi, T., and Itoh, H. Spontaneous nucleation of monodisperse silver halide particles from homogeneous gelatin solution i: silver chloride. Colloids and Surfaces A: Physicochemical and Engineering Aspects, 164 (2-3):183 - 203, 2000. Cited on pages 103 and 104.

Taylor, T. D. and Acrivos, A. On the deformation and drag of a falling viscous drop at low Reynolds number. Journal of Fluid Mechanics, 18:466-476, 1964. Cited on page 61 .

Telford, J. W. A new aspect of coalescence theory. Journal of Meteorology, 12:436 444, 1955. Cited on page 11. 
Tokuyama, M. and Enomoto, Y. Theory of phase-separation dynamics in quenched binary mixtures. Physical Review E, 47:1156-1179, 1993. Cited on page 45.

Toramaru, A. and Maeda, K. Mass and style of eruptions in experimental geysers. Journal of Volcanology and Geothermal Research, 257(0):227 - 239, 2013. Cited on page 1 .

Tölle, M. H. and Krueger, S. K. Effects of entrainment and mixing on droplet size distributions in warm cumulus clouds. Journal of Advances in Modeling Earth Systems, 6(2):281-299, 2014. Cited on page 1.

Vogel, J. The influence of stirring and time-dependent driving on the formation of precipation. Bachelor's thesis, Georg-August-Universität Göttingen, 2013. Cited on pages 13 and 99.

Vollmer, D., Strey, R., and Vollmer, J. Oscillating phase separation in microemulsions. I. Experimental observation. The Journal of Chemical Physics, 107(9):3619-3626, 1997. Cited on page 6 .

Vollmer, J. Phase separation under ultraslow cooling: Onset of nucleation. The Journal of Chemical Physics, 129(16):164502-8, 2008. Cited on pages 4 and 5.

Vollmer, J., Auernhammer, G. K., and Vollmer, D. Minimal model for phase separation under slow cooling. Physical Review Letters, 98(11):115701, 2007. Cited on pages $6,21,70$, and 71 .

Vollmer, J. and Vollmer, D. Cascade nucleation in the phase separation of amphiphilic mixtures. Faraday Discussions, 112:51-62, 1999. Cited on pages 6 and 21.

Vollmer, J., Papke, A., and Rohloff, M. Ripening and focusing of aggregate size distributions with overall volume growth. Frontiers in Physics, 2(18):18, 2014. Cited on pages $13,29,39,45$, and 74 .

Voorhees, P. W. The theory of Ostwald ripening. Journal of Statistical Physics, 38 (1):231-252, 1985. Cited on page 8 .

Wagner, C. Theorie der Alterung von Niederschlägen durch Umlösen (OstwaldReifung). Zeitschrift für Elektrochemie, 65:581-591, 1961. Cited on page 8.

Walker, J. S. and Vause, C. A. Wiederkehrende Phasen. Spektrum der Wissenschaft, page $86 \mathrm{ff}, 1987$. Cited on page 4 .

Wallace, J. M. and Hobbs, P. V. Atmospheric Science - An Introductory Survey, volume 92 of International Geophysics Series. Academic Press, Burlington, MA, 2006. Cited on pages 2, 43, and 107.

Weast, R. C. CRC Handbook of Chemistry and Physics. CRC Press, Inc. Boca Raton, Florida, $69^{\text {th }}$ edition, 1988-1989. Cited on page 117.

Weidauer, T., Pauluis, O., and Schumacher, J. Cloud patterns and mixing properties in shallow moist Rayleigh-Bénard convection. New Journal of Physics, 12(10): 105002, 2010. Cited on page 109. 
Wilkinson, M. A test-tube model for rainfall. EPL (Europhysics Letters), 106(4): 40001, 2014. Cited on pages 13, 18, 74, 76, and 86.

Winkel, F. On Turbulent Rayleigh-Bénard Convection in a Two-Phase Binary Gas Mixture. PhD thesis, Georg-August-Universität, Göttingen, 2014. Cited on page 109.

Woods, A. W. Turbulent plumes in nature. Annual Review of Fluid Mechanics, 42 (1):391-412, 2010. Cited on pages 2 and 110 .

Wylie, J. J., Voight, B., and Whitehead, J. A. Instability of magma flow from volatile-dependent viscosity. Science, 285(5435):1883-1885, 1999. Cited on page 1.

Yao, J. H., Elder, K. R., Guo, H., and Grant, M. Theory and simulation of Ostwald ripening. Physical Review B, 47:14110-14125, 1993. Cited on page 29.

Yelash, L. V. and Kraska, T. On closed-loop liquid-liquid immiscibility. Physical Chemistry Chemical Physics, 1:307-311, 1999. Cited on page 4.

Yeomans, J. M. Statistical mechanics of phase transitions. Oxford University Press, 1992. Cited on page 1.

Yin, Y. and Alivisatos, A. P. Colloidal nanocrystal synthesis and the organic-inorganic interface. Nature, 437:664 - 670, 2005. Cited on page 43.

Zhang, Y. Dynamics of $\mathrm{CO}_{2}$-driven lake eruptions. Nature, 379:57 - 59, 1996. Cited on page 1.

Zhang, Y. and Kling, G. W. Dynamics of lake eruptions and possible ocean eruptions. Annual Review of Earth and Planetary Sciences, 34(1):293-324, 2006. Cited on pages 1 and 110 . 

This work is licensed under the Creative Commons Attribution-NonCommercialNoDerivatives 4.0 International Licence. To view a copy of this license visit http: //creativecommons.org/licenses/by-nc-nd/4.0/. 